\title{
TWRS Privatization Phase I \\ Waste Charácterization Data \\ Evaluation for the Request for Proposal
}

G. K. Patello

K. D. Wiemers

September 1996

Prepared for the U:S. Department of Energy under Contract DE-AC06-76RLO 1830

Pacific Northwest National Laboratory

Operated for the U.S. Department of Energy

by Battelle
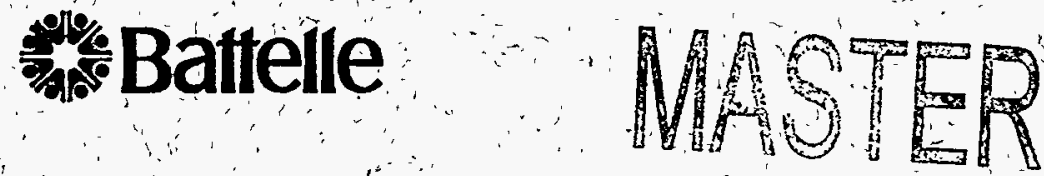

OTRIBUTION OF THIS DOCUMENT IS UNLIMTIEO 


\section{DISCLAIMER}

This report was prépared as an account of work sponsored by an agency of the United States Government. Neither the United States Government nor any agency thereof, nor Battelle Memorial Institute, nor any of their employees, makes any warranty, express or implied, or assumes any legal liability or responsibility for the accuracy, completeness, or úsefulness of any information, apparatus, product, or process disclosed, or represents that its use would not infringe privately owned rights. Reference herein to any, specific commercial product, process, or service by trade name, trademark, manufacturer, or otherwise does not necessárily constitute or imply its endorsement, Jecommendation, or favoring by the United States Government or any agency thereof; or Battelle Memorial institute. The views and opinions of authors expressed herein do not necessarily state or reflect those of the United States Government or àny agency thereof.

\section{PACIFIC NORTHWEST NATIOONAL LABORÁTORY operated by \\ BÄTTELLE \\ for the \\ UNITED STATES DEPARTMENT OF ENERGY under Contract DE-ACO6-76RLO 1830}

Printed in the United States of America

Available to DOE and DOE contractors from the Office of Ścientific and Tèchnićcal Infórmátion, P.O. Box 62, Oak Ridge; TN 37831; prices available from (615) 576-8401:

Available to the public from the National Technical Information Service, U.S. Department of Commerce, 5285 Port Royal Rd., Springfield, VA 22161 


\section{TWRS Privatization Phase I Waste Characterization Data Evaluation for the Request for Proposal}

Authors

GK Patello and KD Wiemers

Technical Contributors

JR FitzPatrick (LANL), DL Herting (WHC), GF Vandegrift (ANL), CJ Conner (ANL), CJ Mertz (ANL), MC Regalbuto (ANL), L Chen (ANL), RL Russell (PNNL)

September 1996

Prepared for the U.S. Department of Energy

under Contract DE-AC06-76RLO 1830

Pacific Northwest National Laboratory

Richland, Washington 99352 



\section{DISCLAIMER}

Portions of this document may be illegible in electronic image products. Images are produced from the best available original document. 


\section{Summary}

Radioactive waste has been stored in large underground tanks at the Hanford Site since 1944. Approximately 212 million liters of waste containing approximately 240,000 metric tons of processed chemical solutions and 177 mega-curies of radionuclides are stored in 177 tanks. These caustic wastes are in the form of liquids, slurries, saltcakes, and sludge. In 1991, the Tank Waste Remediation System (TWRS) Program was established to manage, retrieve, treat, immobilize, and dispose of these wastes in a safe, environmentally sound, and cost-effective manner.

The Department of Energy (DOE) believes that it is feasible to privatize portions of the TWRS Program. Under the Privatization strategy embodied in the request for proposal (RFP); DOE will purchase services from a contractor-owned, contractor-operated facility under a fixedprice type of contract.

Phase I of the TWRS Privatization strategy is a proof-of-concept/commercial demonstrationscale effort. The objectives of Phase I are to 1) demonstrate the technical and business viability of using privatized facilities to treat Hanford tank waste; 2) define and maintain required levels of radiological, nuclear, process, and occupational safety; maintain environmental protection and compliance; and 3) substantially reduce life-cycle costs and time required to treat tank waste.

The TWRS Privatization Support Project Contract Support Team was responsible for preparing the RFP for Phase I. The Waste Characterization Subtask of the TWRS Privatization Support project was charged with collecting and evaluating all information relevant to characterization of candidate wastes for the low-activity waste (LAW) facility feeds. From the waste characterization data current through August 1995; an inventory was created in the form of minimum, maximum, best estimate for 12 chemical analytes and 6 radionuclides. Maximums only were determined for another 23 chemical analytes. The inventory is referred to as the Privatization inventory, and it provides a basis for the RFP LAW feed specification (Patello et al. 1996).

The method used to achieve the objective of producing a technically reviewed inventory is describe by the following steps.

- the waste type selection criteria were established

- the tanks that contain representative waste types were identified

- analytes and radionuclides for which information was to be collected were selected

- $\quad$ reports containing the waste characterization information were gathered

- information was collected into a database

- waste transfer data for each tank were gathered and relevant sampling events were determined

- a new database containing only relevant reviewed waste characterization data was created

- minimum, maximum, and best estimate values were determined for 12 chemical analytes and 6 radionuclides. Maximums only were determined for another 23 chemical analytes

Organic speciation and physical properties were gathered for the tank wastes evaluated and may be found in the individual tank section of this document. Reference reviews and tank volume and sampling summary graphs are also provided for each tank. 
• 


\section{Acronyms}

\begin{tabular}{|c|c|}
\hline AGING & Aging Waste (Neutralized Current Acid Waste [NCAW]) \\
\hline ANL & Argonne National Laboratory \\
\hline CASS & Computer Automated Surveillance System \\
\hline $\mathrm{CC}$ & Complexant Concentrate \\
\hline $\mathrm{CP}$ & Concentrated Phosphate \\
\hline CRQL & Contract Required Quantitation Limit \\
\hline CWHT & Concentrated Waste Holding Tank \\
\hline DC & Dilute Complexed \\
\hline DF & Decontamination Factor \\
\hline $\mathrm{DN}$ & Dilute Non-complexed \\
\hline DOE & Department of Energy \\
\hline DQO & Data Quality Objectives \\
\hline DRCVR & Dilute Receiver \\
\hline DSC & Differential Scanning Calorimetry \\
\hline DSS & Double-Shelled Slurry \\
\hline DSSF & Double-Shelled Slurry Feed \\
\hline DST & Double-Shelled Tank \\
\hline FIC & Food Instrument Company \\
\hline GRTFD & Grout Feed Tank \\
\hline HTCE & Historical Tank Content Estimate \\
\hline ICF KH & ICF Kaiser Hanford Company -- referred to as Kaiser \\
\hline LANL & Los Alamos National Laboratory \\
\hline LAW & Low-Activity Waste \\
\hline NCAW & Neutralized Current Acid Waste \\
\hline NCPLX & Non-Complexed Waste \\
\hline NCRW & Neutralized Cladding Removal Waste \\
\hline PA & Performance Assessment \\
\hline PNNL & Pacific Northwest National Laboratory \\
\hline PUREX & Plutonium/Uranium Extraction Process \\
\hline RFP & Request for Proposal \\
\hline SST & Single-Shelled Tank \\
\hline STIV & Source Term Inventory Validation \\
\hline TBP & Tributyl Phosphate \\
\hline TCD & Tank Characterization Database \\
\hline TCR & Tank Characterization Reports \\
\hline TCRC & Tank Characterization Resource Center \\
\hline TGA & Thermogravimetric Analysis \\
\hline TIC & Total Inorganic Carbon; also refers to carbonate $\left(\mathrm{CO}_{3}^{-2}\right)$ \\
\hline TOC & Total Organic Carbon \\
\hline TPA & Tri-Party Agreement \\
\hline TRAC & Track Radioactive Components \\
\hline TRU & Transuranics \\
\hline TWRS & Tank Waste Remediation System \\
\hline WHC & Westinghouse Hanford Company \\
\hline
\end{tabular}





\section{Contents}

Summary $\ldots \ldots \ldots \ldots \ldots \ldots \ldots \ldots \ldots \ldots \ldots \ldots \ldots \ldots \ldots$ iii

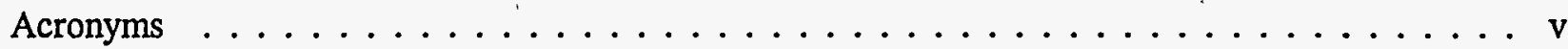

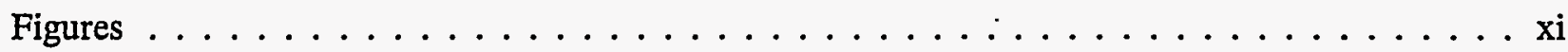

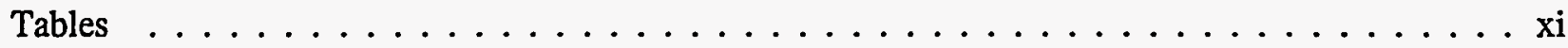

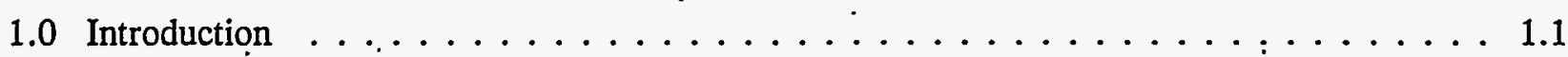

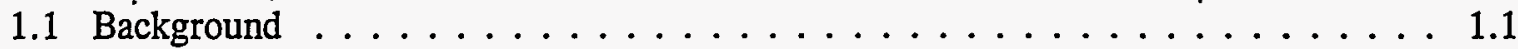

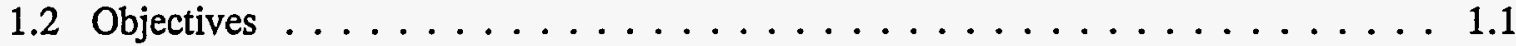

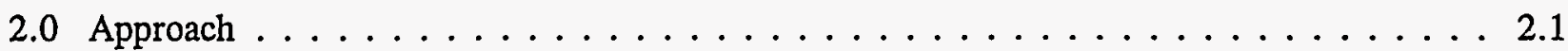

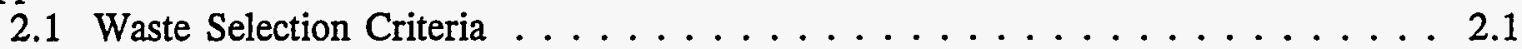

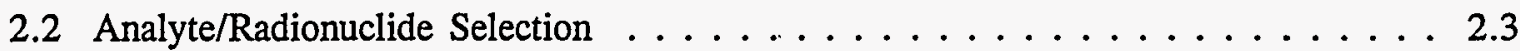

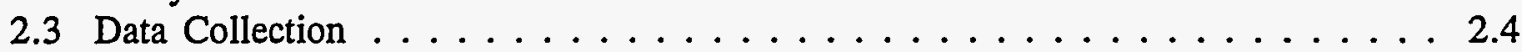

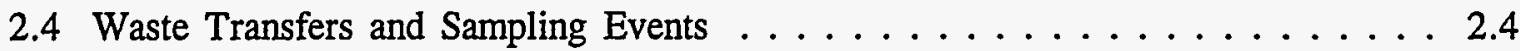

2.5 Data Evaluation . . . . . . . . . . . . . . . . . 2.9

2.6 Final Privatization Database . . . . . . . . . . . . . . 2.11

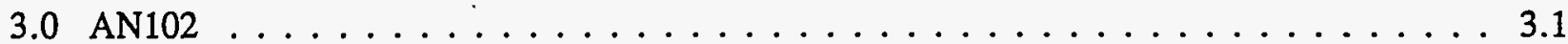

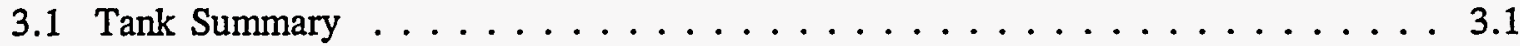

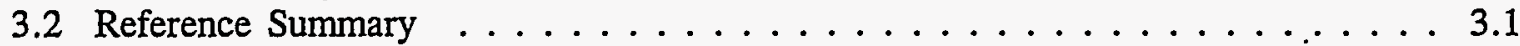

3.3 Waste Volume and Sampling Events . . . . . . . . . . . . . 3.1

3.4 Master Set . . . . . . . . . . . . . . . . . . . . 3.1

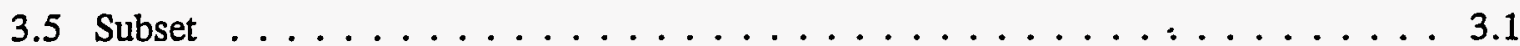

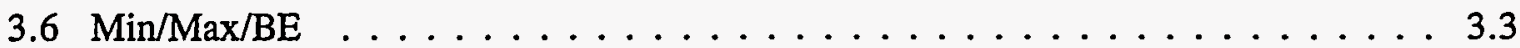

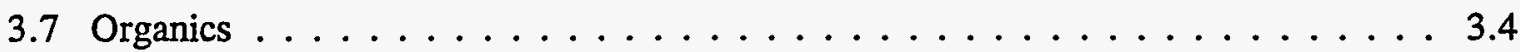

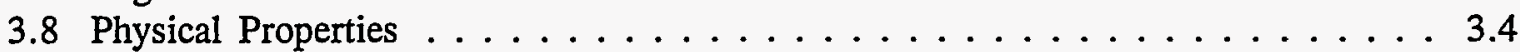

3.9 Limitations/Concerns $\ldots \ldots \ldots \ldots \ldots \ldots \ldots \ldots \ldots \ldots$

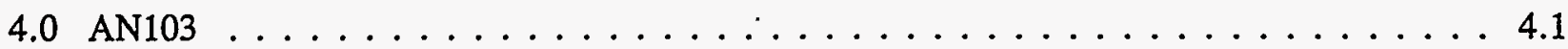

4.1 Tank Summary . . . . . . . . . . . . . . . . . 4.1

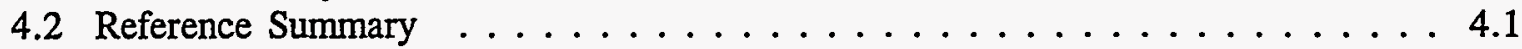

4.3 Waste Volume and Sampling Events . . . . . . . . . . . . . 4.1

4.4 Master Set/Subset . . . . . . . . . . . . . . . . . . . . . 4.4

4.5 Physical Properties . . . . . . . . . . . . . . . . . 4.4

4.6 Limitations/Concerns . . . . . . . . . . . . . . . . . 4.5

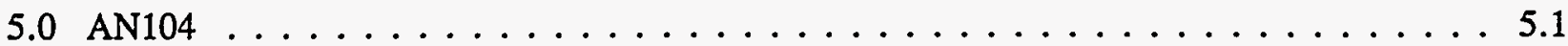

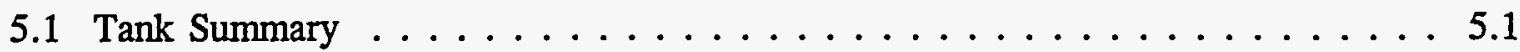

5.2 Reference Summary . . . . . . . . . . . . . . . . 5.1

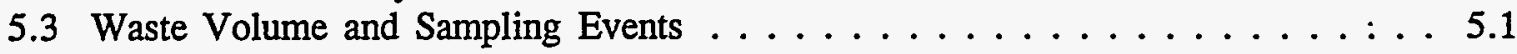

5.4 Master Set/Subset . . . . . . . . . . . . . . . . . . . . 5.1 


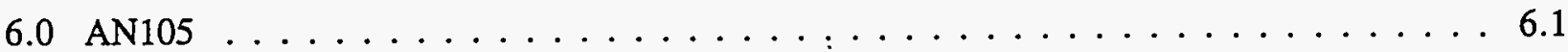

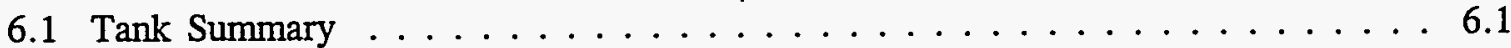

6.2 Reference Summary . . . . . . . . . . . . . . . . . 6.1

6.3 Waste Volume and Sampling Events ... . . . . . . . . . . . 6.1

6.4 Master Set/Subset . . . . . . . . . . . . . . . . . . . 6.1

6.5 Limitations/Concerns . . . . . . . . . . . . . . . 6.1

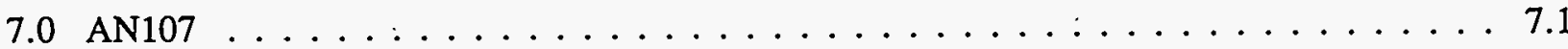

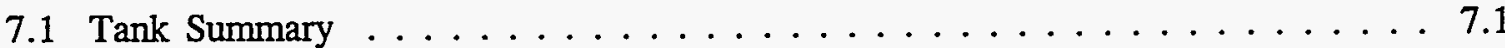

7.2 Reference Summary . . . . . . . . . . . . . . . . . 7.1

7.3 Waste Volume and Sampling Events . . . . . . . . . . . . . . 7.1

7.4 Master Set . . . . . . . . . . . . . . . . . . . . 7.1

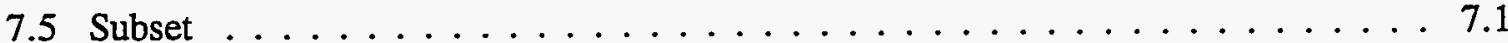

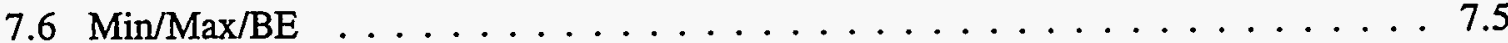

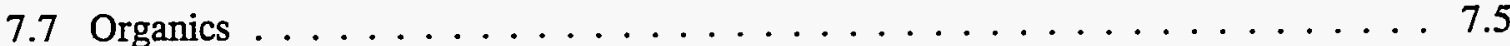

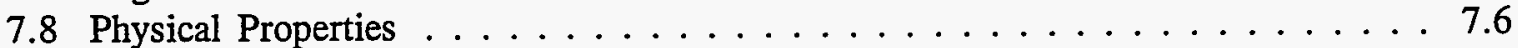

7.9 Limitations/Concerns . . . . . . . . . . . . . . 7.6

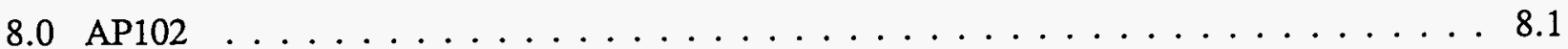

8.1 Tank Summary . . . . . . . . . . . . . . . . . . 8.1

8.2 Reference Summary . . . . . . . . . . . . . . . . . 8.1

8.3 Waste Volume and Sampling Events ... . . . . . . . . . . 8.1

8.4 Master Set . . . . . . . . . . . . . . . . . . . . . . 8.1

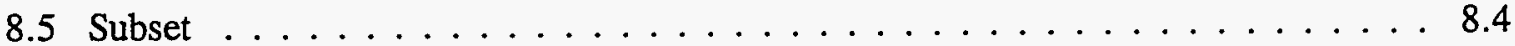

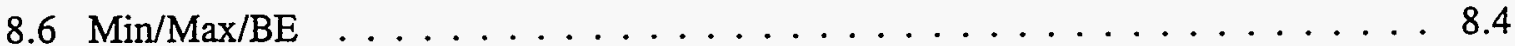

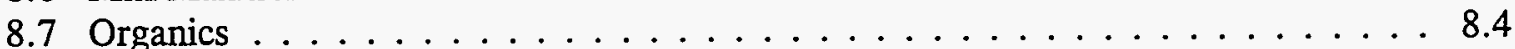

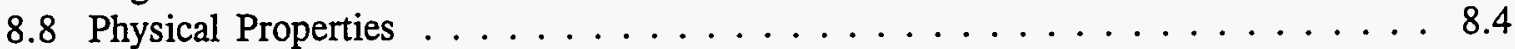

8.9 Limitations/Concerns . . . . . . . . . . . . . . . . 8.7

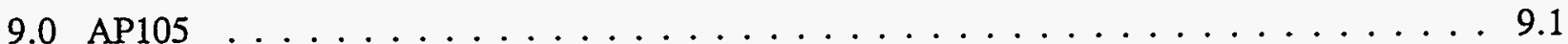

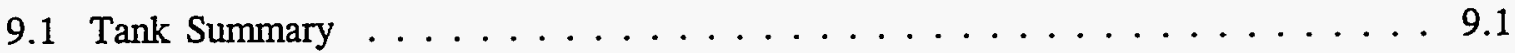

9.2 Reference Summary . . . . . . . . . . . . . . . . . . 9.1

9.3 Waste Volume and Sampling Events ... . . . . . . . . . . . . 9.1

9.4 Master Set . . . . . . . . . . . . . . . . . . . . . . . 9.3

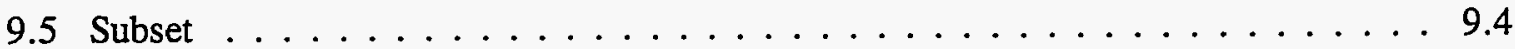

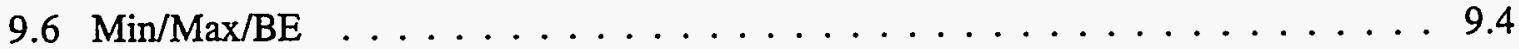

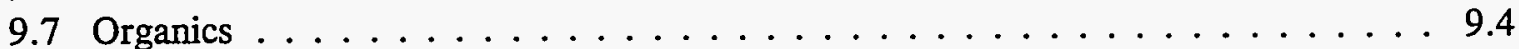

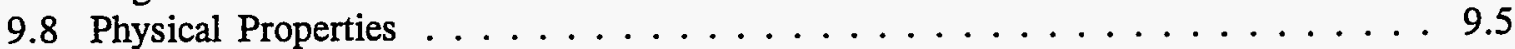

9.9 Limitations/Concerns . . . . . . . . . . . . . . . . . . 9.5

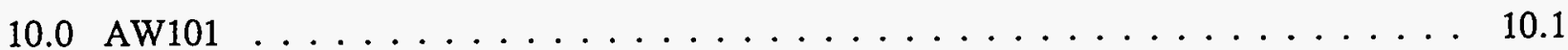

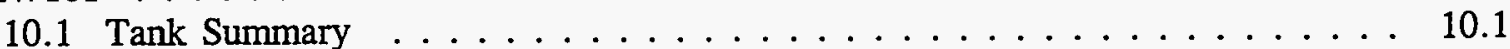

10.2 Reference Summary . . . . . . . . . . . . . . . . . . 10.1

10.3 Waste Volume and Sampling Events . . . . . . . . . . . . . . 10.1

10.4 Master Set . . . . . . . . . . . . . . . . . . . . . . . . . . . . . . . . . . . . . . . . . . . . . .

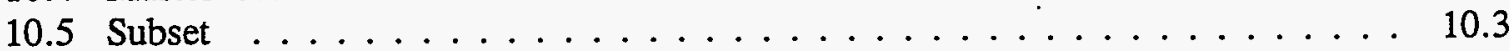


$10.6 \mathrm{Min} / \mathrm{Max} / \mathrm{BE} \ldots \ldots \ldots \ldots \ldots \ldots \ldots \ldots \ldots \ldots \ldots \ldots$

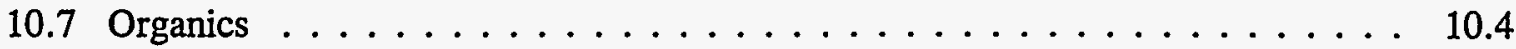

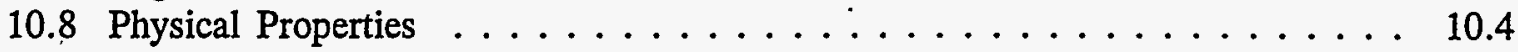

10.9 Limitations/Concerns . . . . . . . . . . . . . . . . . 10.4

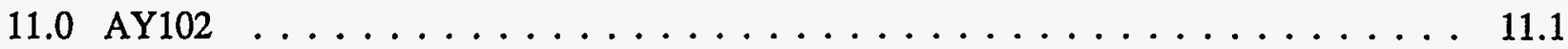

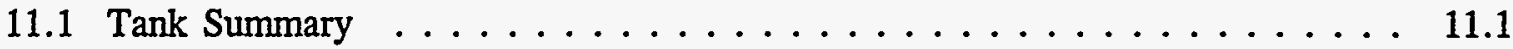

11.2 Reference Summary . . . . . . . . . . . . . . . . . 11.1

11.3 Waste Volume and Sampling Events $\ldots \ldots \ldots \ldots \ldots \ldots \ldots \ldots \ldots$

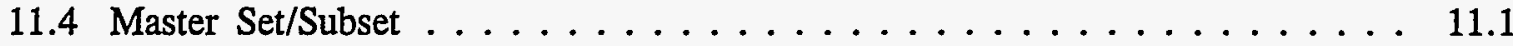

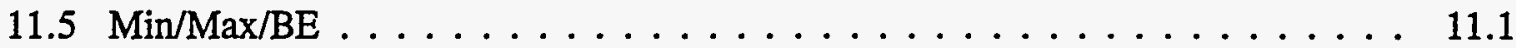

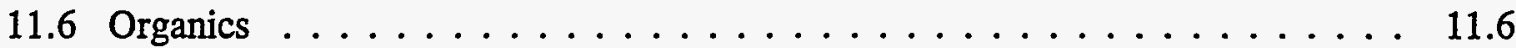

11.7 Physical Properties $\ldots \ldots \ldots \ldots \ldots \ldots \ldots \ldots \ldots \ldots \ldots \ldots \ldots$

11.8 Limitations/Concerns $\ldots \ldots \ldots \ldots \ldots \ldots \ldots \ldots \ldots \ldots \ldots$

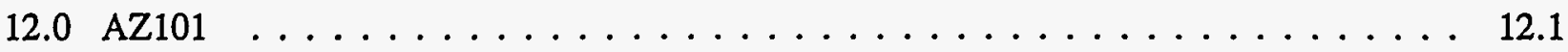

12.1 Tank Summary $\ldots \ldots \ldots \ldots \ldots \ldots \ldots \ldots \ldots \ldots \ldots \ldots \ldots \ldots \ldots$

12.2 Reference Summary . . . . . . . . . . . . . . . . 12.1

12.3 Waste Volume and Sampling Events $\ldots \ldots \ldots \ldots \ldots \ldots \ldots \ldots . \ldots \ldots$

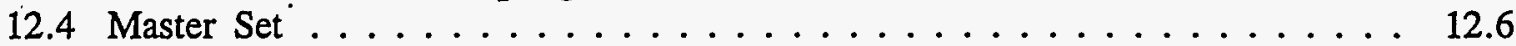

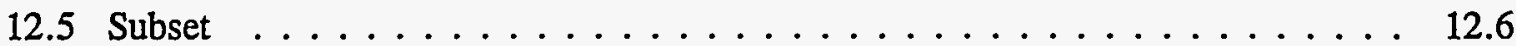

$12.6 \mathrm{Min} / \mathrm{Max} / \mathrm{BE} \ldots \ldots \ldots \ldots \ldots \ldots \ldots \ldots \ldots \ldots \ldots \ldots \ldots \ldots \ldots$

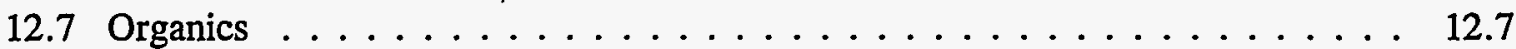

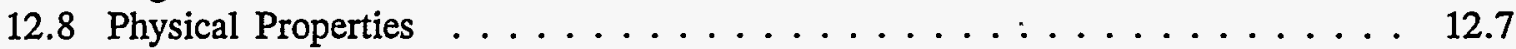

12.9 Limitations/Concerns $\ldots \ldots \ldots \ldots \ldots \ldots \ldots \ldots \ldots \ldots$

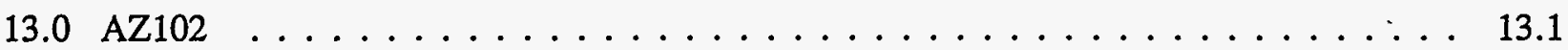

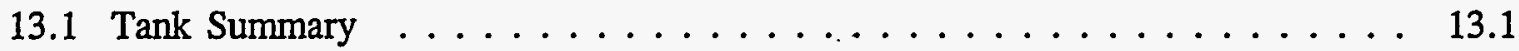

13.2 Reference Summary . . . . . . . . . . . . . . . 13.1

13.3 Waste Volume and Sampling Events $\ldots \ldots \ldots \ldots \ldots \ldots \ldots \ldots \ldots . \ldots \ldots$

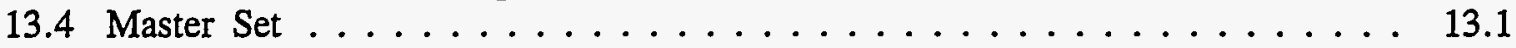

13.5 Subset $\ldots \ldots \ldots \ldots \ldots \ldots \ldots \ldots \ldots \ldots \ldots \ldots \ldots \ldots \ldots \ldots \ldots \ldots .13 .1$

$13.6 \mathrm{Min} / \mathrm{Max} / \mathrm{BE} \ldots \ldots \ldots \ldots \ldots \ldots \ldots \ldots \ldots \ldots \ldots \ldots \ldots$

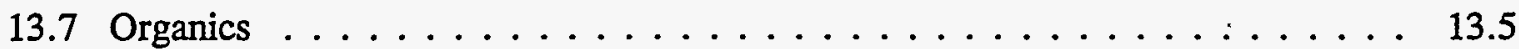

13.8 Physical Properties $\ldots \ldots \ldots \ldots \ldots \ldots \ldots \ldots \ldots \ldots \ldots \ldots$

13.9 Limitations/Concerns $\ldots \ldots \ldots \ldots \ldots \ldots \ldots \ldots . \ldots \ldots$

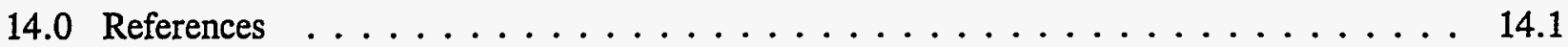

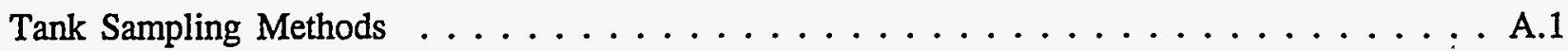

Draft Tank Transfer Report from RS Goheen $\ldots \ldots \ldots \ldots \ldots \ldots \ldots \ldots$. . . . . .

Technical Reviewer Assignments $\ldots \ldots \ldots \ldots \ldots \ldots \ldots \ldots \ldots \ldots$. . . . . . 


\section{Figures}

3.1. Tank Waste Volume and Sampling Event Graph for Tank AN102 . . . . . . . . . 3.3

4.1. Tank Waste Volume and Sampling Event Graph for Tank AN103 . . . . . . . . . . 4.4

5.1. Tank Waste Volume and Sampling Event Graph for Tank AN104 . . . . . . . . . 5.5

6.1. Tank Waste Volume and Sampling Event Graph for Tank AN105 . . . . . . . . . 6.3

7.1. Tank Waste Volume and Sampling Event Graph for Tank AN107 . . . . . . . . . . 7.5

8.1. Tank Waste Volume and Sampling Event Graph for Tank AP102 . . . . . . . . . 8.3

9.1. Tank Waste Volume and Sampling Event Graph for Tank AP105 . . . . . . . . . . 9.3

10.1. Tank Waste Volume and Sampling Event Graph for Tank AW101 . . . . . . . . 10.3

11.1. Tank Waste Volume and Sampling Event Graph for Tank AY102 from 1971 to 1980

11.2. Tank Waste Volume and Sampling Event Graph for Tank AY102 from 1980 to 1999

12.1. Tank Waste Volume and Sampling Event Graph for AZ101 . . . . . . . . . . . 12.6

13.1. Tank Waste Volume and Sampling Event Graph for Tank AZ102 . . . . . . . . 13.5

\section{Tables}

2.1. Technical Issues and Challenges Associated with Waste Envelopes and Waste Types . . 2.2

2.2. Comparison of Sources Identifying Data Needs . . . . . . . . . . . 2.5

2.3. Master Table of Minimum, Maximum, and Best Estimate Values . . . . . . . . 2.12

3.1. Summary of Source Documents Containing Waste Characterization Data for AN102 . . 3.2

4.1. Summary of Source Documents Containing Waste Characterization Data for AN103 . . 4.2

5.1. Summary of Source Documents Containing Waste Characterization Data for AN104 . . 5.2

6.1. Summary of Source Documents Containing Waste Characterization Data for AN105 . . 6.2

7.1. Summary of Source Documents Containing Waste Characterization Data for AN107 . . 7.2

8.1. Summary of Source Documents Containing Waste Characterization Data for AP102 _ . 8.2

8.2. Semivolatile and Volatile Organic Compounds . . . . . . . . . . . 8.5

9.1. Summary of Source Documents Containing Waste Characterization Data for AP105 . . 9.2

10.1. Summary of Source Documents Containing Waste Characterization Data for AW101 • 10.2

11.1. Summary of References Containing Waste Characterization Data for AY102 _ . . . 11.2

12.1. Summary of Source Documents Containing Waste Characterization Data for AZ101 - 12.2

13.1. Summary of Source Documents Containing Waste Characterization Data for AZ102 • 13.2

B.1. Summary of Transfer Information $\ldots \ldots \ldots \ldots \ldots \ldots$

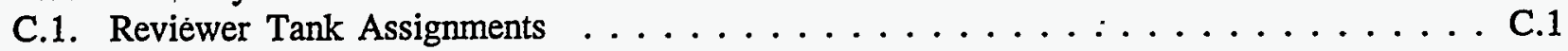




\subsection{Introduction}

\subsection{Background}

Radioactive waste has been stored in large underground storage tanks at the Hanford Site since 1944. Approximately 212 million liters of waste containing approximately 240,000 metric tons of processed chemical solutions and 177 mega-curies of radionuclides are stored in 177 tanks. These caustic wastes are in the form of liquids, slurries, saltcakes, and sludge. In 1991, the Tank Waste Remediation System (TWRS) Program was established to manage, retrieve, treat, immobilize, and dispose of these wastes in a safe, environmentally sound, and cost-effective manner.

The Department of Energy (DOE) believes that it is feasible to privatize portions of the TWRS Program. Under the Privatization strategy embodied in the request for proposal'(RFP), DOE will purchase services from a contractor-owned, contractor-operated facility under a fixedprice type of contract.

Phase I of the TWRS Privatization strategy is a proof-of-concept/commercial demonstrationscale effort. The objectives of Phase I are to 1) demonstrate the technical and business viability of using privatized facilities to treat Hanford tank waste; 2) define and maintain required levels of radiological, nuclear, process, and occupational safety; maintain environmental protection and compliance; and 3) substantially. reduce life-cycle costs and time required to treat Hanford tank waste.

Phase II of the TWRS Privatization strategy is full-scale production, in which the facilities are configured so that the remaining waste from the single-shelled tanks (SST) can be processed by 2018. The objectives of Phase II are to 1) implement the lessons learned from Phase I; 2) process all tank waste into forms suitable for final disposal; 3) achieve price competition and cost savings and 4) meet or exceed the Tri-Party Agreement (TPA) benchmark performance milestones.

\subsection{Objectives}

The TWRS Privatization Support Project Contract Support Team was responsible for preparing the RFP for Phase I. The Waste Characterization Subtask of the TWRS Privatization Support project was charged with collecting and assessing all information relevant to characterization of candidate wastes for the low-activity waste (LAW) facility feeds. This information provided a basis for the RFP LAW feed specification. The waste characterization data was to be presented in the form of best estimate and range for each analyte, radionuclide, and physical property of interest. 


\subsection{Approach}

The process of collecting waste characterization information, evaluating the information, determining the best estimate and range, and defining the RFP feed specifications are described in this document and two companion documents; TWRS Privatization Support Project Waste Characterization Database Development (Johnson 1996) and Low-Activity Waste Envelopes Definition for the TWRS Privatization Phase I Request for Proposal (Patello et al. 1996). The steps of the process were the following:

- $\quad$ establishing the waste type selection criteria

- $\quad$ identifying the tanks that contain representative waste types

- $\quad$ selecting analytes and radionuclides for which information was to be collected

- gathering reports containing the waste characterization information

- collecting information into a database

- $\quad$ gathering waste transfer data for each tank and determining relevant sampling events

- creating a new database containing only relevant reviewed waste characterization data

- determining a best estimate and range for each chemical component and each radionuclide

- $\quad$ preparing summary descriptions of analyses for organic constituents and physical properties, if available

- determining maximum limits for the RFP feed specifications

This document describes the methods used to derive the minimum, maximum, and best estimate compositional values from analytical data for representative double-shelled tank (DST) wastes.

\subsection{Waste Selection Criteria} selection:

Five technology challenges identified by TWRS provided the primary basis for waste
- throughput capacity
- high activity environment
- minor component impact on waste volume
- $\quad$ organic interference with radionuclide removal
- TRU carry-over

Waste types selected to provide a test case for each technology challenge are identified in Table 2.1. During the information gathering process, it became apparent that specifying treatment of waste feed from individual tanks would not be a feasible contractual mechanism. Waste composition changes continually as a result of tank farm operations, and the waste composition data include a large uncertainty, particularly when they are provided for individual tank wastes. Therefore, the strategy for the RFP was revised to include three low-activity waste facility feed envelopes. Each envelope specified an upper limit for constituents believed to be relevant to the 
Table 2.1. Technical Issues and Challenges Associated with Waste Envelopes and Waste Types

\begin{tabular}{|c|c|c|c|c|c|}
\hline Issue & Challenge & Uncertainty & Envelope & $\begin{array}{l}\text { Waste } \\
\text { Type }\end{array}$ & $\begin{array}{l}\text { Tank } \\
\text { FY95 }\end{array}$ \\
\hline \multirow[t]{4}{*}{$\begin{array}{l}\text { Production } \\
\text { Throughput }\end{array}$} & \multirow[t]{4}{*}{$\begin{array}{l}\text { Demonstrate the capability to run } \\
\text { a radioactive facility with a } \\
\text { proven process. }\end{array}$} & & \multirow[t]{4}{*}{ A } & DSSF & $\begin{array}{l}\text { AW101 } \\
\text { AN104 } \\
\text { AN105 } \\
\text { AN106 } \\
\text { AP105 }\end{array}$ \\
\hline & & & & DSS & $\overline{\text { AN103 }}$ \\
\hline & & & & . CP & AP102 \\
\hline & & & & $\mathrm{DN}$ & AP104 \\
\hline $\begin{array}{l}\text { Minor } \\
\text { Components }\end{array}$ & $\begin{array}{l}\text { Demonstrate the capability to } \\
\text { maintain production capacity when } \\
\text { waste-loading of final product is } \\
\text { limited by minor components. }\end{array}$ & & B & NCAW & $\begin{array}{l}\text { AZ101 } \\
\text { AZ102 }\end{array}$ \\
\hline $\begin{array}{l}\text { Cesium } \\
\text { Removal }^{(1)}\end{array}$ & $\begin{array}{l}\text { Demonstrate the capability to } \\
\text { operate a proven process at high } \\
\text { radiation levels and/or to remove } \\
\text { Cs with a proven process. }\end{array}$ & & B & NCAW & $\begin{array}{l}\text { AZ101 } \\
\text { AZ102 }\end{array}$ \\
\hline $\begin{array}{l}\text { High-Activity } \\
\text { Environment }\end{array}$ & $\begin{array}{l}\text { Demonstrate the capability to } \\
\text { operate with high radiation/ heat } \\
\text { levels during waste processing. }\end{array}$ & & B & NCAW & $\begin{array}{l}\text { AZ101 } \\
\text { AZ102 }\end{array}$ \\
\hline $\begin{array}{l}\text { Organic } \\
\text { Impact }\end{array}$ & $\begin{array}{l}\text { Demonstrate the capability to } \\
\text { develop and design an organic } \\
\text { destruction process that will } \\
\text { decomplex the waste when the } \\
\text { degree of destruction required is } \\
\text { unknown. }\end{array}$ & $\begin{array}{l}\text { Degree of destruction } \\
\text { required is unknown. } \\
\text { No process has been } \\
\text { demonstrated }\end{array}$ & $\mathrm{C}$ & $\mathrm{CC}$ & $\begin{array}{l}\text { AN102 } \\
\text { AN107 }\end{array}$ \\
\hline \multirow[t]{2}{*}{$\begin{array}{l}\text { TRU } \\
\text { Carryover }\end{array}$} & \multirow[t]{2}{*}{$\begin{array}{l}\text { Demonstrate the capability to } \\
\text { separate solids. }\end{array}$} & \multirow{2}{*}{$\begin{array}{l}\text { Effect of particle size } \\
\text { on rétrieval. } \\
\text { Undemonstrate } \\
\text { technology. }\end{array}$} & & NCRW & $\begin{array}{l}\text { AW103 } \\
\text { AW105 }\end{array}$ \\
\hline & & & & NCPLX & C109 \\
\hline
\end{tabular}

(1) No longer an issue associated with Envelope B.

technical challenges. The envelope limits were derived from both technology constraints and an assessment of data and inventory estimates for groupings of waste types (Patello et al. 1996).

Envelope A represents waste that will test the production capacity and fission product removal efficiency of the plants while producing a final product in which the waste loading will be limited by sodium. Envelope B waste is similar to Envelope A; except that the waste loading in the final product will be limited by minor component concentrations. Cesium removal was initially envisioned as an issue/challenge for envelope $\mathrm{B}$, however, the LAW specification has changed from an allowable cesium- 137 concentration of $1 \mathrm{Ci} / \mathrm{m}^{3}$ to $3 \mathrm{Ci} / \mathrm{m}^{3}$ in the final waste product. Therefore, the estimated decontamination factor (DF) requirements are reduced to such a degree that it is unnecessary to have an envelope devoted to the treatment of waste with cesium- 137 decontamination requirements beyond the range covered by Envelope A (McKee et al. 1995). The private contractor will, however, be challenged by working in a high activity/high heat 
environment. Envelope $\mathrm{C}$ represents waste with complexing agents that may interfere with strontium-90 and/or transuranic (TRU) decontamination, and therefore, require demonstration of organic destruction or another acceptable mitigation technology. Envelope D represents noncomplexed waste that requires strontium-90 and/or TRU decontamination by solid/liquid separation to prevent strontium-90 and/or TRU carryover into the final product. This envelope included less than $1 \%$ of the DST and SST waste, was listed by McKee (1995) as optional, and was not included in the final RFP (DOE 1996).

Based on the technical issues, 16 tanks were selected in May 1995 for waste characterization data analysis: AN102, AN103, AN104, AN105, AN106, AN107, AW101, AW103, AW105, AP102, AP104, AP105, AY102, AZ101, AZ102, and C109.

ICF Kaiser Hanford Company (Kaiser). was chosen to collect the waste characterization data for 14 tanks $^{(\mathrm{a})}$ because they had collected data for the Historical Tank Content Estimates (HTCE) (Brevick, Gaddis, and Pickett 1995) and for the Tank Waste Source Term Inventory Validation report (STIV) (Brevick, Gaddis, and Johnson 1995). For the STIV report, Kaiser collected analytical data from the Tank Characterization Resource Center (TCRC), Tank Characterization Database (TCD), Tank Characterization Reports (TCR), Braun database, and Wastren database; and inventory estimates from HTCE, and Track Radioactive Components (TRAC). The data were collected for all 177 tanks but were limited to 24 chemical analytes and 11 radionuclides. The database for the STIV report provided a starting point for the Privatization waste characterization effort.

\subsection{Analyte/Radionuclide Selection}

The Privatization waste characterization subtask required 35 chemical analytes and 16 radionuclides. Selection of the analytes and radionuclides was based on known process technology and the needs for that technology as identified in a document describing data needs ${ }^{(\mathfrak{b})}$. Information from end-user staff, internal communications ${ }^{(\mathbf{c})}$, and the draft TWRS Privatization Data Quality Objectives $(\mathrm{DQO})^{(\mathrm{d})}$ also addressed the need for characterization of specific analytes and radionuclides. Table 2.2 summarizes the contributions from these documents and the reasons for selecting each analyte or radionuclide, and lists the analytes and radionuclides included in the RFP. The analytes and radionuclides listed in the RFP envelope specifications are limited to those for

(a) AN103 and AY102 were added to the list of tanks in Envelope A after the statement of work was issued to Kaiser. Technical reviewers at Argonne National Laboratory collected and evaluated the analytical data for these tanks.

(b) Vandegrift GF. 1995. Vendor Needs for Waste Characterization. Attachment 1 in Data Requirements for TWRS Privatization Waste Characterization. WHC-SD-WM-DQO-023, Rev. O (Draft), Westinghouse Hanford Company, Richland, Washington.

(c) Kovach JL. 1995. Draft Listing of Analytes and Properties of Concern for the TWRS Privatization Effort. Draft Report dated 10/21/95, Pacific Northwest National Laboratory, Richland, Washington.

(d) Wiemers KD, JW Hunt, GF Vandegrift, and J Sedlet. 1995. Data Requirements for TWRS Privatization Waste Characterization. WHC-SD-WM-DQO-023, Rev. O (Draft), Westinghouse Hanford Company, Richland, Washington. 
Table 2.2. Comparison of Sources Identifying Data Needs

\begin{tabular}{|c|c|c|c|c|c|c|c|}
\hline $\begin{array}{l}\text { STIV } \\
\text { Data } \\
\text { (a) }\end{array}$ & $\begin{array}{l}\text { Privatization } \\
\text { Data } \\
\text { Collected } \\
\text { (b) }\end{array}$ & $\begin{array}{l}\text { Data Needs } \\
\text { Vandegrift } \\
\text { (c) }\end{array}$ & $\begin{array}{l}\text { Data Needs } \\
\text { Kovach } \\
\text { (d) }\end{array}$ & $\begin{array}{c}\text { DQO } \\
\text { (e) }\end{array}$ & $\begin{array}{l}\text { Kupfer } \\
\text { Site-wide } \\
\text { inventory } \\
\text { (f) }\end{array}$ & $\begin{array}{c}\text { RFP } \\
\text { (g) }\end{array}$ & Justification \\
\hline & & ${ }^{3} \mathrm{H}$ & & ${ }^{3} \mathrm{H}$ & ${ }^{3} \mathrm{H}$ & & $1,3,4,5,6,7,8,10$ \\
\hline & ${ }^{14} \mathrm{C}$ & ${ }^{14} \mathrm{C}$ & ${ }^{14} \mathrm{C}$ & ${ }^{14} \mathrm{C}$ & ${ }^{14} \mathrm{C}$ & & $1,3,4,5,6,7,8,10$ \\
\hline & & & & & $59 \mathrm{Ni}$ & & $3,4,6,8$ \\
\hline \multirow[t]{4}{*}{${ }^{60} \mathrm{Co}$} & ${ }^{60} \mathrm{Co}$ & & & ${ }^{60} \mathrm{Co}$ & ${ }^{60} \mathrm{Co}$ & & $1,3,4,5,6,7$ \\
\hline & & ${ }^{63} \mathrm{Ni}$ & & & ${ }^{63} \mathrm{Ni}$ & & $3,4,5,6,8$ \\
\hline & ${ }^{-79} \mathrm{Se}$ & & ${ }^{79} \mathrm{Se}$ & ${ }^{79} \mathrm{Se}$ & ${ }^{79} \mathrm{Se}$ & & $1,3,4,5,6,7,8$ \\
\hline & & & $87 \mathrm{Rb}$ & & & & $3,4,6,8$ \\
\hline${ }^{89190} \mathrm{Sr}$ & ${ }^{90} \mathrm{Sr}^{*}$ & ${ }^{90} \mathrm{Sr}$ & ${ }^{90} \mathrm{Sr}$ & ${ }^{90} \mathrm{Sr}$ & ${ }^{90} \mathrm{Sr} / \mathrm{Y}$ & ${ }^{90} \mathrm{Sr}$ & $1,3,4,5,6,7,8$ \\
\hline \multirow[t]{9}{*}{${ }^{90} \mathrm{Y}$} & ${ }^{90} \mathrm{Y}$ & & & & & & $1,3,4,5,6,7,8$ \\
\hline & & & ${ }^{93} \mathrm{Zr}$ & ${ }^{93} \mathrm{Zr}$ & ${ }^{93} \mathrm{Zr}$ & & $1,3,4,5,6,7,8$ \\
\hline & ${ }^{99} \mathrm{Tc} *$ & ${ }^{99} \mathrm{Tc}$ & ${ }^{99} \mathrm{Tc}$ & ${ }^{99} \mathrm{Tc}$ & ${ }^{99} \mathrm{Tc}$ & ${ }^{99} \mathrm{Tc}$ & $1,3,4,5,6,7,8$ \\
\hline & & & ${ }^{106} \mathrm{Ru}$ & ${ }^{106} \mathrm{Ru} / \mathrm{Rh}$ & ${ }^{106} \mathrm{Ru}$ & & $1,3,4,5,6,7,8$ \\
\hline & & & & & ${ }^{113} \mathrm{Cd} / \mathrm{m}$ & & $3,4,8$ \\
\hline & & & & & ${ }^{125} \mathrm{Sb}$ & & $3,4,8$ \\
\hline & ${ }^{126} \mathrm{Sn}$ & & ${ }^{126} \mathrm{Sn}$ & ${ }^{126} \mathrm{Sn}$ & ${ }^{126} \mathrm{Sn}$ & & $1,3,4,5,6,7,8$ \\
\hline & ${ }^{129} \mathrm{I}$ & ${ }^{129} \bar{I}$ & ${ }^{129} \mathrm{~T}$ & ${ }^{129} \mathrm{~T}$ & ${ }^{129} \mathrm{I}$ & & $1,3,4,5,6,7,8$ \\
\hline & & & ${ }^{134} \mathrm{Cs}$ & & ${ }^{134} \mathrm{Cs}$ & & $3,4,5,8$ \\
\hline \multirow[t]{5}{*}{$\overline{137} \mathrm{Cs}$} & ${ }^{137} \mathrm{Cs}^{*}$ & ${ }^{137} \mathrm{Cs}$ & ${ }^{137} \mathrm{Cs}$ & ${ }^{137} \mathrm{Cs}$ & ${ }^{137} \mathrm{Cs} / \mathrm{Ba}$ & ${ }^{137} \mathrm{Cs}$ & $1,3,4,5,6,7,8$ \\
\hline & & & ${ }^{144} \mathrm{Ce}$ & ${ }^{144} \mathrm{Ce} / \mathrm{Pr}$ & & & $1,3,4,5,6,7,8$ \\
\hline & & & ${ }^{147} \mathrm{Pm}$ & & & & $3,4,8$ \\
\hline & & & & & ${ }^{151} \mathrm{Sm}$ & & $3,4,6,8$ \\
\hline & & & & ${ }^{152} \mathrm{Eu}$ & ${ }^{152} \mathrm{Eu}$ & & $1,3,4,5,6,7,8$ \\
\hline \multirow[t]{10}{*}{${ }^{154} \mathrm{Eu}$} & ${ }^{154} \mathrm{Eu}$ & & & ${ }^{154} \mathrm{Eu}$ & ${ }^{154} \mathrm{Eu}$ & & $1,3,4,5,6,7,8$ \\
\hline & & & & & ${ }^{155} \mathrm{Eu}$ & & $1,3,4,7,8$ \\
\hline & & & & & ${ }^{226} \mathrm{Ra}$ & & $3,4,6,8$ \\
\hline & & & & & ${ }^{277} \mathrm{Ac}$ & & $1,3,4,6,7,8$ \\
\hline & & & & & ${ }^{228} \mathrm{Ra}$ & & $3,4,6,8$ \\
\hline & & & & & ${ }^{299} \mathrm{Th}$ & & $3,4,6,8$ \\
\hline & & & & & ${ }^{23 !} \mathrm{Pa}$ & & $1,3,4,7,8$ \\
\hline & & & ${ }^{232} \mathrm{Th}$ & ${ }^{232} \mathrm{Th}$ & ${ }^{232} \mathrm{Th}$ & & $1,3,4,5,6,7,8$ \\
\hline & & & & & ${ }^{232} \mathrm{U}$ & & $3,4,6,8$ \\
\hline & & & & & ${ }^{233} \mathrm{U}$ & & $1,3,4,6,7,8$ \\
\hline
\end{tabular}




\begin{tabular}{|c|c|c|c|c|c|c|c|}
\hline $\begin{array}{c}\text { STIV } \\
\text { Data } \\
\text { (a) }\end{array}$ & $\begin{array}{l}\text { Privatization } \\
\text { Data } \\
\text { Collected } \\
\text { (b) }\end{array}$ & $\begin{array}{c}\text { Data Needs } \\
\text { Vandegrift } \\
\text { (c) }\end{array}$ & $\begin{array}{c}\text { Data Needs } \\
\text { Kovach } \\
\text { (d) }\end{array}$ & $\begin{array}{c}\text { DQO } \\
\text { (e) }\end{array}$ & $\begin{array}{c}\text { Kupfer } \\
\text { Site-wide } \\
\text { inventory } \\
\text { (f) }\end{array}$ & $\begin{array}{c}\text { RFP } \\
\text { (g) }\end{array}$ & Justification \\
\hline & & & ${ }^{234} \mathrm{U}$ & ${ }^{234} U$ & ${ }^{234} \mathrm{U}$ & & $1,3,4,5,6,7,8$ \\
\hline & & & ${ }^{235} \mathrm{U}$ & ${ }^{235} \mathrm{U}$ & ${ }^{235} \mathrm{U}$ & & $1,3,4,5,6,7,8$ \\
\hline - & & & ${ }^{236} \mathrm{U}$ & ${ }^{236} \mathrm{U}$ & ${ }^{236} \mathrm{U}$ & & $1,3,4,5,6,7,8$ \\
\hline${ }^{237} \mathrm{~Np}$ & ${ }^{237} \mathrm{~Np}^{*}$ & ${ }^{237} \mathrm{~Np}$ & ${ }^{237} \mathrm{~Np}$ & ${ }^{237} \mathrm{~Np}$ & ${ }^{237} \mathrm{~Np}$ & & $1,3,4,5,6,7,8$ \\
\hline & & & ${ }^{238} \mathrm{U}$ & ${ }^{238} \mathrm{U}$ & ${ }^{238} \mathrm{U}$ & & $1,3,4,5,6,7,8$ \\
\hline${ }^{238} \mathrm{Pu}$ & ${ }^{238} \mathrm{Pu}$ & & ${ }^{238} \mathrm{Pu}$ & ${ }^{238} \mathrm{Pu}$ & ${ }^{238} \mathrm{Pu}$ & & $1,3,4,5,6,7,8$ \\
\hline${ }^{239 / 240} \mathrm{Pu}$ & ${ }^{239 / 240} \mathrm{Pu}^{*}$ & ${ }^{239} \mathrm{Pu}$ & $239 / 240 \mathrm{Pu}$ & $2391240 \mathrm{Pu}$ & $239 / 240 \mathrm{Pu}$ & & $1,3,4,5,6,7,8$ \\
\hline${ }^{241} \mathrm{Am}$ & ${ }^{241} \mathrm{Am}^{*}$ & $.241 \mathrm{Am}$ & ${ }^{241} \mathrm{Am}$ & ${ }^{241} \mathrm{Am}$ & ${ }^{241} \mathrm{Am}$ & & $1,3,4,5,6,7,8$ \\
\hline${ }^{24 !} \mathrm{Pu}$ & ${ }^{24 I} \mathrm{Pu}$ & & ${ }^{241} \mathrm{Pu}$ & ${ }^{241} \mathrm{Pu}$ & ${ }^{241} \mathrm{Pu}$ & . & $1,3,4,5,6,7,8$ \\
\hline & & & ${ }^{242} \mathrm{Pu}$ & ${ }^{242} \mathrm{Pu}$ & ${ }^{242} \mathrm{Pu}$ & & $1,3,4,5,6,7,8$ \\
\hline & & & & & ${ }^{242} \mathrm{Cm}$ & & $1,3,4,5,6,7,8$ \\
\hline & & & ${ }^{243} \mathrm{Am}$ & ${ }^{243} \mathrm{Am}$ & ${ }^{243} \mathrm{Am}$ & & $1,3,4,5,6,7,8$ \\
\hline & & & ${ }^{243} \mathrm{Cm}$ & ${ }^{243} \mathrm{Cm}$ & ${ }^{243} \mathrm{Cm}$ & & $1,3,4,5,6,7,8$ \\
\hline${ }^{244} \mathrm{Cm}$ & ${ }^{244} \mathrm{Cm}$ & & ${ }^{244} \mathrm{Cm}$ & ${ }^{244} \mathrm{Cm}$ & ${ }^{244} \mathrm{Cm}$ & & $1,3,4,5,6,7,8$ \\
\hline & & & & & total alpha & & $1,7,6,10$ \\
\hline & & & , & & total beta & & 6,10 \\
\hline & & & $\mathrm{Ag}$ & $\mathrm{Ag}$ & $\mathrm{Ag}$ & & $3,4,5,6,8$ \\
\hline & $\mathrm{Al}^{*}$ & $\mathrm{Al} / \mathrm{Al}(\mathrm{OH})_{4}^{-}$ & $\mathrm{Al}$ & $\mathrm{Al}$ & Al & $\mathrm{Al}$ & $.2,3,4,5,6$ \\
\hline & As & & As & As & As & & $3,4,5,6,8$ \\
\hline & & . & B & B & B & & $3,4,5,6,8$ \\
\hline & $\mathrm{Ba}$ & & $\mathrm{Ba}$ & $\overline{\mathrm{Ba}}$ & $\mathrm{Ba}$ & $\mathrm{Ba}$ & $2,5,8$ \\
\hline & $\mathrm{Be}$ & & & $\mathrm{Be}$ & $\mathrm{Be}$ & & $3,4,5,6,8$ \\
\hline & & & $\mathrm{Bi}$ & $\mathrm{Bi}$ & $\mathrm{Bi}$ & & $2,3,4,5,6,8$ \\
\hline & $\mathrm{Ca}$ & & $\mathrm{Ca}$ & $\mathrm{Ca}$ & $\mathrm{Ca}$ & $\mathrm{Ca}$ & $2,3,4,5,6$ \\
\hline & $\mathrm{Cd}$ & & $\mathrm{Cd}$ & Cd & $\mathrm{Cd}$ & $\mathrm{Cd}$ & $3,4,5,6,8$ \\
\hline & $\mathrm{Ce}$ & & Rare Earths & $\mathrm{Ce}$ & $\mathrm{Ce}$ & . & 3 \\
\hline & $\mathrm{Cl}^{*}$ & $\mathrm{Cl}$ & $\mathrm{Cl}$ & $\mathrm{Cl}$ & $\mathrm{Cl}$ & $\mathrm{Cl}$ & $2,3,4,5,6,8,9$ \\
\hline & $\mathrm{CN}^{-}$ & & & & $\mathrm{FeCN} / \mathrm{CN}$ & & $8^{\circ}$ \\
\hline & Co & & $\mathrm{Co}$ & Co & Co & & 8 \\
\hline & $\begin{array}{c}\mathrm{Cr} * / \mathrm{Cr}(\mathrm{III}) \\
/ \mathrm{Cr}(\mathrm{VI})\end{array}$ & $\mathrm{Cr}$ & $\mathrm{Cr} / \mathrm{Cr}(\mathrm{VI})$ & $\mathrm{Cr}$ & $\begin{array}{c}\mathrm{Cr} / \mathrm{Cr}(\mathrm{III}) / \\
\mathrm{Cr}(\mathrm{VI})\end{array}$ & $\mathrm{Cr}$ & $2,3,4,5,6,8$ \\
\hline & & Cs & Cs & Cs & Cs & & 2 \\
\hline & & & $\mathrm{Cu}$ & $\mathrm{Cu}$ & $\mathrm{Cu}$ & & $\cdot 8$ \\
\hline & $\mathrm{F}$ & $\bar{F}$ & $F$ & $F$ & F & $F$ & $2,3,4,5,6,8$ \\
\hline
\end{tabular}




\begin{tabular}{|c|c|c|c|c|c|c|c|}
\hline $\begin{array}{l}\text { STIV } \\
\text { Data } \\
\text { (a) }\end{array}$ & $\begin{array}{l}\text { Privatization } \\
\text { Data } \\
\text { Collected } \\
\text { (b) }\end{array}$ & $\begin{array}{l}\text { Data Needs } \\
\text { Vandegrift } \\
\text { (c) }\end{array}$ & $\begin{array}{l}\text { Data Needs } \\
\text { Kovach } \\
\text { (d) }\end{array}$ & $\begin{array}{r}\text { DQO } \\
\text { (e) }\end{array}$ & $\begin{array}{c}\text { Kupfer } \\
\text { Site-wide } \\
\text { inventory } \\
\text { (f) }\end{array}$ & $\begin{array}{c}\text { RFP } \\
\text { (g) }\end{array}$ & Justification \\
\hline & $\mathrm{Fe}^{*}$ & & $\mathrm{Fe}$ & $\mathrm{Fe}$ & $\mathrm{Fe}$ & $\mathrm{Fe}$ & $3,4,5,6,8$ \\
\hline & & & & $\mathrm{H}_{2} \mathrm{O}$ & & & 8 \\
\hline & $\mathrm{Hg}$ & & $\mathrm{Hg}$ & $\mathrm{Hg}$ & $\mathrm{Hg}$ & $\mathrm{Hg}$ & $3,4,5,6,8$ \\
\hline & $\mathrm{K}^{*}$ & $\mathbf{K}$ & $\mathbf{K}$ & K & $\mathrm{K}$ & $\mathrm{K}$ & $2,3,4,5,6$ \\
\hline & $\mathrm{La}$ & & Rare Earths & $\mathrm{La}$ & $\mathrm{La}$ & $\mathrm{Ia}$ & 3 \\
\hline & & & $\mathrm{Li}$ & $\mathrm{Li}$ & $\mathrm{Li}$ & & $2,3,4,5,6$ \\
\hline & & & $\mathrm{Mg}$ & $\mathrm{Mg}$ & $\mathrm{Mg}$ & & $2,3,4,5,6$ \\
\hline & & & & Mn & $\mathrm{Mn}$ & & $2,3,4,5,6,8$ \\
\hline & & & Mo & & Mo & & $3,4,8$ \\
\hline & $\mathrm{Na}^{*}$ & $\mathrm{Na}$ & $\mathrm{Na}$ & $\mathrm{Na}$ & $\mathrm{Na}$ & & $2,3,4,5,6$ \\
\hline & Nd & & Rare Earths & Nd & Nd & & 3 \\
\hline & $\mathrm{NH}_{3}$ & $\mathrm{NH}_{3}$ & $\mathrm{NH}_{3}$ & $\mathrm{NH}_{3} / \mathrm{NH}_{4}^{+}$ & $\mathrm{NH}_{4}^{+}$ & & $2,3,4,8$ \\
\hline & $\mathrm{Ni}$ & & $\mathrm{Ni}$ & $\mathrm{Ni}$ & $\mathrm{Ni}$ & $\mathrm{Ni}$ & $2,3,4,5,6,8$ \\
\hline & $\mathrm{NO}_{2}^{-*}$ & $\mathrm{NO}_{2}^{-}$ & $\mathrm{NO}_{2}^{-}$ & $\mathrm{NO}_{2}^{-}$ & $\mathrm{NO}_{2}^{-}$ & $\mathrm{NO}_{2}^{-}$ & $2,3,4,5,6,8,9$ \\
\hline & $\mathrm{NO}_{3}^{-*}$ & $\mathrm{NO}_{3}$ & $\mathrm{NO}_{3}^{-}$ & $\mathrm{NO}_{3}^{-}$ & $\mathrm{NO}_{3}^{-}$ & $\mathrm{NO}_{3}^{-}$ & $2,3,4,5,6,8,9$ \\
\hline & $\mathrm{OH}^{*} *$ & $\overline{\mathrm{OH}^{-}}$ & & $\mathrm{OH}^{-}$ & $\overline{\mathrm{OH}}$ & $\overline{\mathrm{OH}^{-}}$ & $2,8,9$ \\
\hline & & & $\mathrm{O}_{2}$ & & & & 2,8 \\
\hline & & $\bar{P}$ & $\bar{P}$ & & & & $3,4,5,6,8$ \\
\hline & $\mathrm{Pb}$ & & $\mathrm{Pb}$ & $\mathrm{Pb}$ & $\mathrm{Pb}$ & $\mathrm{Pb}$ & $3,4,5,6,8$ \\
\hline & & & Pd & & $\mathrm{Pd}$ & & 3,4 \\
\hline & $\mathrm{PO}_{4}^{-3 *}$ & $\mathrm{PO}_{4}^{-3} / \mathrm{HPO}_{4}^{-}$ & & $\mathrm{PO}_{4}^{-3}$ & $\mathrm{PO}_{4}^{-3}$ & $\mathrm{PO}_{4}^{-3}$ & $2,3,4,5,6,8$ \\
\hline & & & $\mathbf{P t}$ & & $\mathbf{P t}$ & & 3,4 \\
\hline & & & $\mathbf{R h}$ & & $\mathrm{Rh}$ & & 3,4 \\
\hline & & & $\mathbf{R u}$ & & $R u$ & & 3,4 \\
\hline & & & $S$ & $\mathrm{~S}$ & & & $3,4,5,6,8$ \\
\hline & $\mathrm{Sb}$ & & $\mathrm{Sb}$ & $\mathrm{Sb}$ & $\mathrm{Sb}$ & & $3,4,5,6,8$ \\
\hline & $\mathrm{Se}$ & & $\mathrm{Se}$ & $\mathrm{Se}$ & $\mathrm{Se}$ & & $3,4,5,6,8$ \\
\hline & & & Si & $\mathrm{Si}$ & $\mathrm{Si}$ & & $3,4,5,6$ \\
\hline & & & Sn & & & & 8 \\
\hline & $\mathrm{SO}_{4}^{-2 *}$ & $\mathrm{SO}_{4}^{-2}$ & $\mathrm{SO}_{4}^{-2}$ & $\mathrm{SO}_{4}^{-2}$ & $\mathrm{SO}_{4}^{-2}$ & $\mathrm{SO}_{4}^{-2}$ & $2,3,4,5,6,8$ \\
\hline & Sr & & $\mathrm{Sr}$ & $\mathrm{Sr}$ & $\mathrm{Sr}$ & & $2,3,4,5,6,8$ \\
\hline & $\mathrm{Te}$ & & $\mathrm{Te}$ & $\mathrm{Te}$ & $\mathrm{Te}$ & & $3,4,5,6,8$ \\
\hline & $\mathrm{Tl}$ & & & $\mathrm{Tl}$ & $\mathrm{Tl}$ & & 3,8 \\
\hline & & $\mathrm{CO}_{3}^{-2}$ & $\mathrm{CO}_{3}^{-2}$ & $\operatorname{TIC}\left(\mathrm{CO}_{3}^{-{ }^{-2}}\right)$ & $\operatorname{TIC}\left(\mathrm{CO}_{3}^{-2}\right)$ & TIC & $2,3,8$ \\
\hline
\end{tabular}




\begin{tabular}{|c|c|c|c|c|c|c|c|}
\hline $\begin{array}{l}\text { STIV } \\
\text { Data } \\
\text { (a) }\end{array}$ & $\begin{array}{l}\text { Privatization } \\
\text { Data } \\
\text { Collected } \\
\text { (b) }\end{array}$ & $\begin{array}{l}\text { Data Needs } \\
\text { Vandegrift } \\
\text { (c) }\end{array}$ & $\begin{array}{l}\text { Data Needs } \\
\text { Kovach } \\
\text { (d) }\end{array}$ & $\begin{array}{c}\text { DQO } \\
\text { (e) }\end{array}$ & $\begin{array}{l}\text { Kupfer } \\
\text { Site-wide } \\
\text { inventory } \\
\text { (f) }\end{array}$ & $\begin{array}{l}\text { RFP } \\
\text { (g) }\end{array}$ & Justification \\
\hline & & $\begin{array}{c}\text { Organic } \\
\text { Complexants }\end{array}$ & $\begin{array}{c}\text { Organic } \\
\text { Complexants }\end{array}$ & $\begin{array}{c}\text { Organic } \\
\text { Complexants }\end{array}$ & & & $2,3,8$ \\
\hline & TOC* & & Organics & TOC & TOC & TOC & $3,4,5,6,8$ \\
\hline & & & & & formate & & $2,3,8$ \\
\hline & & oxalate & & & oxalate & & $2,3,8$ \\
\hline & $\bar{U}$ & & $\overline{\mathrm{U}}$ & $\overline{\mathrm{U}}$ & $\bar{U}$ & & $3,4,5,6,7,8$ \\
\hline & $\mathrm{V}$ & & & $\mathrm{V}$ & $\mathrm{V}$ & & 8,10 \\
\hline & & & & & $\overline{\mathrm{W}}$ & & 8,10 \\
\hline & & & $\overline{Z n}$ & $\mathrm{Zn}$ & $\mathrm{Zn}$ & & $3,4,5,6,8$ \\
\hline & & & $\mathrm{Zr}$ & $\mathrm{Zr}$ & $\mathrm{Zr}$ & & $3,4,5,6$ \\
\hline
\end{tabular}

*Major component in the Privatization database.

a) Brevick et al. 1995.

b) Johnson 1996 .

c) Vandegrift GF. 1995. Vendor Needs for Waste Characterization. Attachment 1 in Data Requirements for TWRS Privatization Waste Characterization. WHC-SD-WM-DQO-023, Rev. O (Draft), Westinghouse Hanford Company, Richland, Washington.

d) Kovach JL. 1995. Draft Listing of Analytes and Properties of Concern for the TWRS Privatization Effort. Draft Report dated 10/21/95, Pacific Northwest National Laboratory, Richland, Washington.

Justification Notes:

Pretreatment

1. Radionuclide removal

2. Affects efficiency of radionuclide removal efficiency. Immobilization

3. Limit waste loading.

4. Affect design of vitrification equipment.

5. Affects waste classification.

6. Potential effect to LAW disposal system performance assessment.

Balance of Plant

7. Plant design, shielding requirements, radiation exposure, criticality, safety, emission control.

8. Toxic, volatile, corrosive, potentially explosive, or flammable; contribute to off gas;

9. Affects corrosion rate of mild steel tanks.

10. Environmental impact documentation. e) Wiemers KD, JW Hunt, GF Vandegrift, and J Sedlet. 1995. Data Requirements for TWRS Privatization Waste Characterization. WHC-SD-WM-DQ0-023, Rev. O (Draft), Westinghouse Hanford Company, Richland, Washington.

f) Kupfer MJ. 1996. Interim Report: Best-Basis Inventories of Chemicals and Radionuclides in Hanford Site Tank Waste. WHC-SD-WM-TI-740, Rev. C-draft, Westinghouse Hanford Company, Richland, Washington.

g) DOE 1996. 
which sufficient information was available to derive a maximum limit.

Of the 35 chemical analytes and 16 radionuclides, a major component set (designated by an asterisk in Table 2.2) of 12 chemical analytes and 6 radionuclides was selected to undergo complete technical review; the remaining (minor) components were reviewed only to determine the maximum value.

\subsection{Data Collection}

The database for the STIV report was the starting point for the Privatization waste characterization effort. Kaiser collected the source documents and identified the additional data for the chemical analytes and radionuclides that were not included in the STIV data set. Kaiser also included newer analytical data (through August 1995) that had not been collected for the STIV. This data came from TCRs, the TCD, or directly from the analytical laboratory. The relationships between different data sources and a description of the sources are provided by Patello and Wiemers (1996).

The data from the STIV were converted from their original Excel spreadsheets to a Paradox database to allow easier searching and retrieval of the data. The data were converted to common units, $\mathrm{Bq} / \mathrm{L}$ and $\mathrm{Ci} / \mathrm{L}$ for radionuclide and $\mu \mathrm{g} / \mathrm{L}$ and $\mathrm{mol} / \mathrm{L}$ for chemical analytes. The radionuclides were also decayed to $12 / 31 / 94$; data collected after this date- were not decayed. Values for neptunium-237, plutonium-239/240, and yttrium-90 were not decayed because their half lives are long. The data assembly which included all data points before review was called the Master Set. The Master Set tables and other information on Kaiser's data collection are reported by Johnson (1996). A review of the source documents for each tank is provided in the tank sections of this report.

\subsection{Waste Transfers and Sampling Events}

Evaluators recognized, early in the data evaluation process, the need to understand waste transfers between tanks and the timing of sampling events with respect to the transfers. Tank volumes, solids volumes, and solids volume measurement dates were obtained from Waste Tank Summary Reports by B.M. Hanlon published monthly. Predicted tank volumes were taken from an internal memo ${ }^{(a)}$, and from Operational Waste Volume Projection by Koreski (1995). Sample dates were gleaned from all available references for each tank, including technical reports and internal memos $^{(b)}$. The graphs (shown in the respective tank sections) describe for each tank:

- $\quad$ capacity

- volume changes over time

- $\quad$ solids volume and the proportion of solids to total volume

- $\quad$ waste types transferred into each tank

(a) Koreski GM. 1995. Double Shell Tank (DST) Transfers. Internal Memo to KD Wiemers dated August 17, 1995, Westinghouse Hanford Company, Richland, Washington.

(b) Stanton, Jr GA. 1996. Baseline Sampling Schedule, Draft 4.8. Internal memo \# 75610-96-09 to JM Appel et al. dated 7/26/96, Westinghouse Hanford Company, Richland, Washington. 
- $\quad$ tanks with inadequate sampling

- $\quad$ sample data that are relevant to the current tank contents

- type of sampling performed (see appendix for descriptions of sampling methods)

- tanks that are still active and their predicted futures.

The volume measurements are current through April 1996. Projected volumes are shown through 1999. Sampling information is provided as either a sampling date or a memo date if no sampling date can be determined; sample type, and, in parentheses, number of samples. Samples dated after July 1996 are planned sample dates and are shown in italics on the graphs.

Almost all waste transfers can be identified on the plot as volume changes. Occasionally transfers may have a canceling effect and show no volume change. Tank transfers are therefore also identified at the top of the plot with the type of waste that was transferred.

\subsection{Data Evaluation}

The primary objective of the data evaluation process was to determine minimum, maximum, and best estimate values for each 'selected component in each tank. This objective was modified during the data evaluation process to decrease the scope of the evaluation process and thereby meet an accelerated schedule. The new objective was to determine minimum, maximum, and best estimate values for major component species (see Table 2.2), and maximum values for the minor components.

As a first step to meeting the objective, the characterization data collected by Kaiser in the Master Set were evaluated with respect to the tank waste volume/sampling information. This revealed as of May 1995 that data for waste in tanks AN104, AN105, AN106, AP104, AW103, AW105, and C109 were not relevant for characterization because sampling had last occurred before a major transfer, and the data did not represent the waste currently in the tank. The only tanks with relevant characterization data were AN102, AN107, AP102, AP105, AW101, AZ101, and AZ102.

It again became necessary to limit the scope of the evaluation to meet an accelerated schedule; the evaluators therefore decided to evaluate only the supernatant characterization data. For Phase I, only the supernatant will be processed and therefore solids data do not need to be evaluated to support the RFP. Solids information will need to be evaluated for Phase II.

The characterization data were evaluated by technical reviewers from Argonne National Laboratory (ANL), Los Alamos National Laboratory (LANL), and Westinghouse Hanford Company - (WHC). The groups received for each tank to be reviewed Master Sets of all solids and liquid data for all selected components (See Appendix $C$ for the reviewer tank assignments). The groups also received original data references (memos, letter reports, analysis reports) so that they could take into consideration all the information concerning a data point. All valid reviewed data were assembled into a database, referred to as the Subset. Data were disqualified from the Subset for the following reasons:

- Braun, Wastren, TRAC, and HTCE data were not original analytical data. (Note HTCE data are inventory estimates only) 
- The sample was taken before a large waste transfer

- The data point was for solids in the tank. The solids data were reviewed to verify that they were not supernatant data.

- The value was a duplicate point

- The value was a less-than $(<)$ value. These values were considered valid if no other data existed for that analyte or radionuclide

- The value could not be traced to a report, memorandum or other reference

- The value was from an evaporator feed sample

- The laboratory reported matrix interference for that particular analyte

- Miscellaneous problems with the sampling or analytical methods as determined by the technical knowledge of the reviewer.

Kaiser assembled the Subset database based on the reviewer's evaluation of the data, and the reviewers checked the Subset for accuracy. The Subset tables are documented by Johnson (1996).

An exception to this data evaluation process was made in the case of AN103, AP102, AY102, and AZ102. For AP102 and AZ102, the reviewers used the original source references to directly create a data Subset for these tanks. They reviewed the data references, disqualified the references that contained out of date data, and included the data from valid references in the Subset. AN103 and AY102 were not included in Kaiser's work scope. Reviewers evaluated AN103 references and found no relevant data. For AY102, the minimum, maximum, and best estimate were created directly from the TCR.

Once the Subsets were finalized, the reviewers determined a minimum, maximum, and best estimate value for all the major component species and a maximum value for the minor component species for each tank. The method of determining these values varied with the information available for the tank waste and was left to the technical discretion of the reviewer. The method used for each tank waste is described in the tank specific sections of this document. A master listing of minimum, maximum, and best estimate values is shown in Table 2.3. This set of minimum, maximum and best estimate values became the Privatization Inventory. Each reviewer also provided an evaluation of the data source references and assembled organic component data and physical property data for their tanks. This information is in the tank specific sections of this document.

\subsection{Final Privatization Database}

The final database including the Subset information as determined by the reviewers is in Paradox, a site-supported database application. The TWRS Privatization Waste Characterization Database is available on the Internet (http://twins.pnl.gov:8001/twrs_rfp/chardata/chardata.htm) in the Paradox format or in Excel spreadsheet format. The database is searchable for tank, analyte, radionuclide etc. 
Table 2.3. Master Table of Minimum, Maximum, and Best Estimate Values

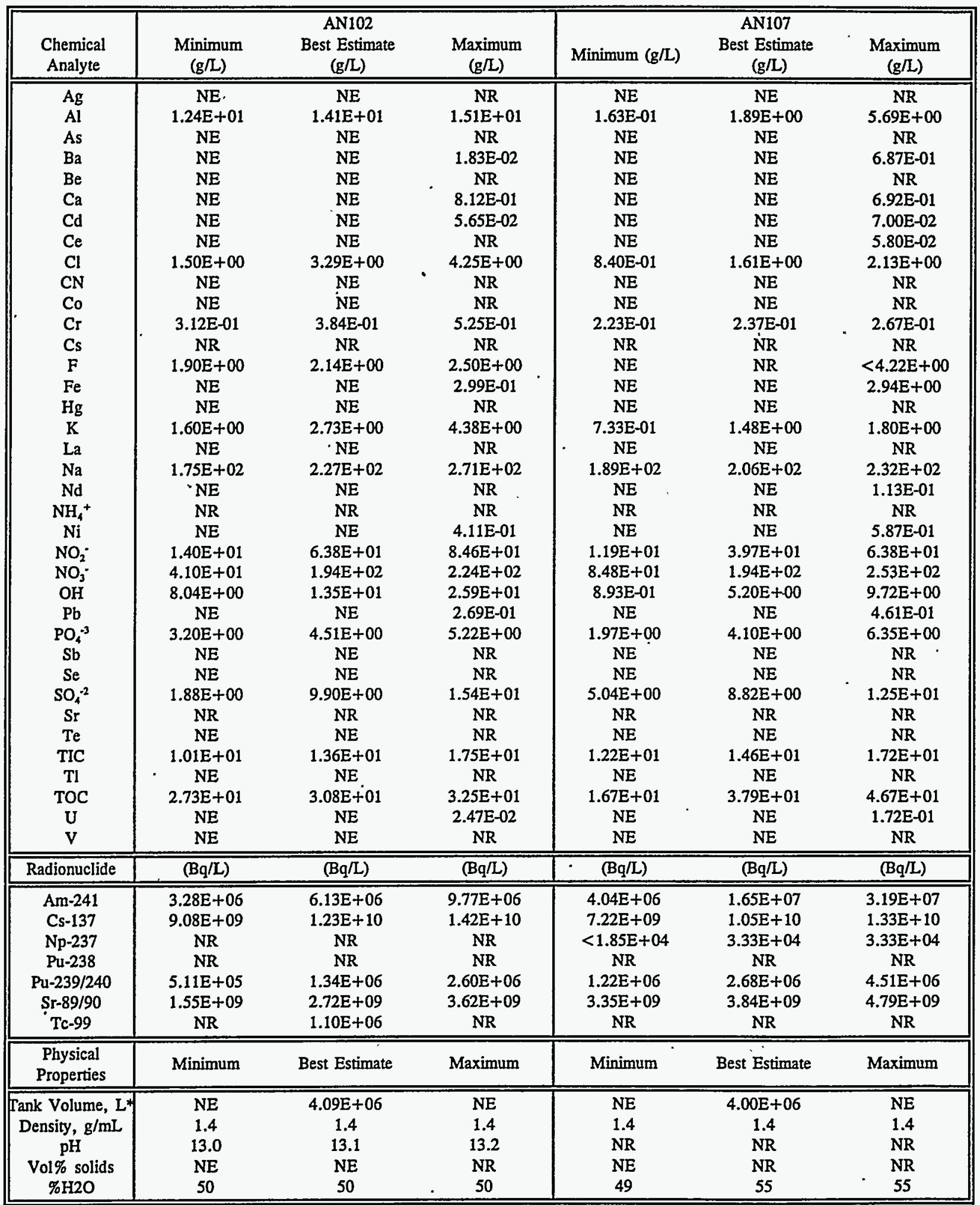

* Volume based on Hanlon 1996 (April, 1996).

NE: Not Estimated

NR: Not Reported in source documents 
Table 2.3. Cont.

\begin{tabular}{|c|c|c|c|c|c|c|}
\hline $\begin{array}{l}\text { Chemical } \\
\text { Analyte }\end{array}$ & $\begin{array}{c}\text { Minimum } \\
(\mathrm{g} / \mathrm{L})\end{array}$ & $\begin{array}{c}\text { AP102 } \\
\text { Best Estimate } \\
(\mathrm{g} / \mathrm{L})\end{array}$ & $\begin{array}{c}\text { Maximum } \\
(\mathrm{g} / \mathrm{L})\end{array}$ & $\begin{array}{c}\text { Minimum } \\
(\mathrm{g} / \mathrm{L})\end{array}$ & $\begin{array}{c}\text { AP105 } \\
\text { Best Estimate } \\
(\mathrm{g} / \mathrm{L})\end{array}$ & $\begin{array}{c}\text { Maximum } \\
(\mathrm{g} / \mathrm{L})\end{array}$ \\
\hline $\mathrm{Ag}$ & NE & NE & $<5.00 \mathrm{E}-03$ & NE & $\mathrm{NE}$ & NR \\
\hline $\mathrm{Al}$ & $1.11 \mathrm{E}+01$ & $1.16 \mathrm{E}+01$ & $1.21 \mathrm{E}+01$ & $9.72 \mathrm{E}+00$ & $1.17 \mathrm{E}+01$ & $1.40 \mathrm{E}+01$ \\
\hline As & NE & NE & $1.02 \mathrm{E}-04$ & NE & NE & $1.03 E-03$ \\
\hline $\mathrm{Ba}$ & $\mathrm{NE}$ & $\mathrm{NE}$ & $4.19 E-04$ & NE & NE & $1.45 \mathrm{E}-03$ \\
\hline $\mathrm{Be}$ & $\mathrm{NE}$ & $\mathrm{NE}$ & $1.68 \mathrm{E}-04$ & NE & NE & $1.68 \mathrm{E}-03$ \\
\hline $\mathrm{Ca}$ & NE & $\mathrm{NE}$ & NR & NE & NE & NR \\
\hline $\mathrm{Cd}$ & NE & NE & $1.79 E-03$ & NE & NE & $2.05 \mathrm{E}-03$ \\
\hline $\mathrm{Ce}$ & NE & $\mathrm{NE}$ & NR & NE & $\mathrm{NE}$ & NR \\
\hline $\mathrm{Cl}$ & $2.36 \mathrm{E}+00$ & $2.86 \mathrm{E}+00$ & $4.56 \mathrm{E}+00$ & $1.14 \mathrm{E}+00$ & $2.36 \mathrm{E}+00$ & $4.07 E+00$ \\
\hline $\mathrm{CN}$ & NE & NE & $2.46 \mathrm{E}-02$ & NE & NE & $1.89 \mathrm{E}-02$ \\
\hline Co & NE & $\mathrm{NE}$ & NR & NE & NE & NR \\
\hline $\mathrm{Cr}$ & $5.91 E-01$ & $6.24 \mathrm{E}-01$ & $7.16 \mathrm{E}-01$ & $1.45 \mathrm{E}-01$ & $1.87 \mathrm{E}-01$ & $2.19 \mathrm{E}-01$ \\
\hline Cs & NR & NR & NR & NR & NR & NR \\
\hline $\mathrm{F}$ & $<1.32 \mathrm{E}-02$ & $<1.66 \mathrm{E}-01$ & $<2.09 \mathrm{E}-01$ & $<1.01 \mathrm{E}-02$ & $1.54 \mathrm{E}+00$ & $8.86 \mathrm{E}+00$ \\
\hline $\mathrm{Fe}$ & NE & NE & $1.05 E-02$ & NE & NE & $9.67 \mathrm{E}-03$ \\
\hline $\mathrm{Hg}$ & NE & NE & $<1.00 \mathrm{E}-05$ & NE & NE & NR \\
\hline $\mathrm{K}$ & $1.19 \mathrm{E}+00$ & $1.30 \mathrm{E}+00$ & $1.40 \mathrm{E}+00$ & $2.83 E+01$ & $3.10 \mathrm{E}+01$ & $3.70 E+01$ \\
\hline $\mathrm{La}$ & NE & NE & NR & NE & NE & NR \\
\hline $\mathrm{Na}$ & $9.80 \mathrm{E}+01$ & $1.02 E+02$ & $1.06 \mathrm{E}+02$ & $1.46 \mathrm{E}+02$ & - $1.67 \mathrm{E}+02$ & $1.93 E+02$ \\
\hline $\mathrm{Nd}$ & NE & $\mathrm{NE}$ & NR & NE & NE & NR \\
\hline $\mathrm{NH}_{4}^{+}$ & $<4.00 \mathrm{E}-02$ & $2.66 \mathrm{E}-01$ & 4.13E-01 & NR & NR & NR \\
\hline $\mathrm{Ni}$ & NE & NE & $3.45 \mathrm{E}-02$ & NE & NE & $1.23 \mathrm{E}-02$ \\
\hline $\mathrm{NO}_{2}^{-}$ & $3.09 \mathrm{E}+01$ & $3.75 \mathrm{E}+01$ & $5.51 \mathrm{E}+01$ & $3.60 \mathrm{E}+01$ & $4.83 E+01$ & $6.23 E+01$ \\
\hline $\mathrm{NO}_{3}^{-}$ & $5.37 \mathrm{E}+01$ & $7.68 \mathrm{E}+01$ & $9.73 E+01$ & $1.25 \mathrm{E}+02$ & $1.65 E+02$ & $2.23 E+02$ \\
\hline $\mathrm{OH}$ & $7.91 E+00$ & $9.07 \mathrm{E}+00$ & $9.52 \mathrm{E}+00$ & $5.31 E+01$ & $5.40 \mathrm{E}+01$ & $5.48 E+01$ \\
\hline $\mathrm{Pb}$ & NE & NE & 5.33E- 03 & NE & NE & $7.62 \mathrm{E}-03$ \\
\hline $\mathrm{PO}_{4}^{-3}$ & $8.09 E+00$ & $1.07 \mathrm{E}+01$ & $1.37 \mathrm{E}+01$ & $2.16 \mathrm{E}-01$ & $4.40 \mathrm{E}-01$ & $6.76 \mathrm{E}-01$ \\
\hline $\mathrm{Sb}$ & NE & NE & $9.47 \mathrm{E}-03$ & NE & NE & $6.76 \mathrm{E}-03$ \\
\hline $\mathrm{Se}$ & NE & NE & $6.79 \mathrm{E}-04$ & NE & NE & $1.95 \mathrm{E}-04$ \\
\hline $\mathrm{SO}_{4}^{-2}$ & $3.54 \mathrm{E}+00$ & $4.18 \mathrm{E}+00$ & $5.17 \mathrm{E}+00$ & $1.13 \mathrm{E}+00$ & $2.42 \mathrm{E}+00$ & $4.88 \mathrm{E}+00$ \\
\hline $\mathrm{Sr}$ & NR & NR & NR & NR & NR & NR \\
\hline $\mathrm{Te}$ & NE & NE & NR & $\mathrm{NE}$ & NE & NR \\
\hline TIC & $4.36 \mathrm{E}+00$ & $5.23 E+00$ & $5.96 \mathrm{E}+00$ & $3.48 \mathrm{E}+00$ & $4.49 \mathrm{E}+00$ & $6.18 \mathrm{E}+00$ \\
\hline $\mathrm{T}$ & NE & NE & NR & NE & NE & NR \\
\hline TOC & $3.00 E+00$ & $3.37 \mathrm{E}+00$ & $5.57 E+00$ & $1.85 \mathrm{E}+00$ & $2.75 \mathrm{E}+00$ & $3.24 E+00$ \\
\hline $\mathrm{U}$ & NE & NE & $7.09 \mathrm{E}-03$ & NE & NE & $4.86 \mathrm{E}-02$ \\
\hline $\mathrm{V}$ & NE & $\mathrm{NE}$ & NR & $\mathrm{NE}$ & NE & NR \\
\hline Radionuclide & $(\mathrm{Bq} / \mathrm{L})$ & $(\mathrm{Bq} / \mathrm{L})$ & $(\mathrm{Bq} / \mathrm{L})$ & $(\mathrm{Bq} / \mathrm{L})$ & $(\mathrm{Bq} / \mathrm{L})$ & $(\mathrm{Bq} / \mathrm{L})^{\circ}$ \\
\hline Am-241 & $7.79 \mathrm{E}+03$ & $1.51 \mathrm{E}+04$ & $3.63 E+04$ & $9.76 \mathrm{E}+03$ & $1.50 \mathrm{E}+04$ & $2.23 E+04$ \\
\hline Cs-137 & $7.23 E+09$ & $8.33 E+09$ & $8.62 E+09$ & $7.07 \mathrm{E}+09$ & $8.28 E+09$ & $9.70 \mathrm{E}+09$ \\
\hline $\mathrm{Np}-237$ & $<8.07 \mathrm{E}+03$ & $3.70 \mathrm{E}+04$ & $3.70 E+04$ & NR & NR & $<1.60 E+04$ \\
\hline Pu-238 & $<3.11 \mathrm{E}+03$ & $<3.91 E+03$ & $<7.68 \mathrm{E}+03$ & NR & NR & $<1.75 E+04$ \\
\hline Pu-239/240 & $<2.44 \mathrm{E}+03$ & $<2.53 \mathrm{E}+03$ & $<2.77 \mathrm{E}+03$ & $4.44 E+03$ & $5.85 E+03$ & $6.70 \mathrm{E}+03$ \\
\hline Sr-89/90 & $2.05 E+07$ & $4.60 \mathrm{E}+07$ & $6.22 \mathrm{E}+07$ & $6.41 E+06$ & $7.37 \mathrm{E}+06$ & $9.22 \mathrm{E}+06$ \\
\hline Tc-99 & $4.70 \mathrm{E}+05$ & $2.84 \mathrm{E}+06$ & $3.77 E+06$ & $2.07 \mathrm{E}+06$ & $2.58 \mathrm{E}+06$ & $3.18 E+06$ \\
\hline $\begin{array}{c}\text { Physical } \\
\text { Properties }\end{array}$ & Minimum & Best Estimate & Maximum & Minimum & Best Estimate & Maximum \\
\hline Tank Volume, L* & NE & $4.15 E+06$ & NE & NE & $3.09 E+06$ & NE \\
\hline Density, g/mL & 1.2 & 1.2 & 1.2 & 1.3 & 1.3 & 1.4 \\
\hline $\mathrm{pH}$ & NR & 13.7 & NR & NR & $>14$ & NR \\
\hline Vol\% solids & NR & NR & NR & NE & NE & NR \\
\hline \% $12 \mathrm{O}$ & 74 & 75 & 76 & 55 & 60 & 65 \\
\hline
\end{tabular}

* Volume based on Hanlon 1996 (April, 1996).

NE: Not Estimated

NR: Not Reported in source documents 
Table 2.3. Cont.

\begin{tabular}{|c|c|c|c|c|c|c|}
\hline $\begin{array}{l}\text { Chemical } \\
\text { Analyte }\end{array}$ & $\begin{array}{l}\text { Minimum } \\
(\mathrm{g} / \mathrm{L})\end{array}$ & $\begin{array}{c}\text { AW101 } \\
\text { Best Estimate } \\
(\mathrm{g} / \mathrm{L})\end{array}$ & $\begin{array}{l}\text { Maximum } \\
(\mathrm{g} / \mathrm{L})\end{array}$ & $\begin{array}{c}\text { Minimum } \\
(\mathrm{g} / \mathrm{L})\end{array}$ & $\begin{array}{c}\text { AY102 } \\
\text { Best Estimate } \\
(\mathrm{g} / \mathrm{L})\end{array}$ & $\begin{array}{c}\text { Maximum } \\
(\mathrm{g} / \mathrm{L})\end{array}$ \\
\hline $\begin{array}{c}\mathrm{Ag} \\
\mathrm{Al} \\
\mathrm{As} \\
\mathrm{Ba} \\
\mathrm{Be} \\
\mathrm{Ca} \\
\mathrm{Cd} \\
\mathrm{Ce} \\
\mathrm{Cl} \\
\mathrm{CN} \\
\mathrm{Co} \\
\mathrm{Cr} \\
\mathrm{Cs} \\
\mathrm{F} \\
\mathrm{Fe} \\
\mathrm{Hg} \\
\mathrm{K} \\
\mathrm{La}^{\mathrm{Na}} \\
\mathrm{Nd}^{+} \\
\mathrm{NH}_{4}^{+} \\
\mathrm{Ni} \\
\mathrm{NO}_{2}^{-} \\
\mathrm{NO}_{3}^{-} \\
\mathrm{OH}^{-} \\
\mathrm{Pb}_{4}^{-3} \\
\mathrm{~Pb}^{-3} \\
\mathrm{Se}^{-2} \\
\mathrm{Sr}_{4}^{-2} \\
\mathrm{Te} \\
\mathrm{TC}_{\mathrm{Tl}} \\
\mathrm{TOC} \\
\mathrm{U} \\
\mathrm{V}\end{array}$ & $\begin{array}{c}\text { NE } \\
2.05 E+01 \\
N E \\
N E \\
N E \\
N E \\
N E \\
N E \\
4.25 E+00 \\
N E \\
N E \\
1.15 E-01 \\
N R \\
N R \\
N E \\
N E \\
2.31 E+01 \\
N E \\
1.59 E+02 \\
N E \\
N R \\
N E \\
6.30 E+01 \\
1.56 E+02 \\
5.66 E+01 \\
N E \\
1.21 E+00 \\
N E \\
N E \\
7.10 E-01 \\
N R \\
N E \\
1.90 E+00 \\
N E \\
2.39 E+00 \\
N E \\
N E \\
N \\
N \\
N \\
N\end{array}$ & $\begin{array}{c}\mathrm{NE} \\
3.49 \mathrm{E}+01 \\
\mathrm{NE} \\
\mathrm{NE} \\
\mathrm{NE} \\
\mathrm{NE} \\
\mathrm{NE} \\
\mathrm{NE} \\
5.73 \mathrm{E}+00 \\
\mathrm{NE} \\
\mathrm{NE} \\
2.38 \mathrm{E}-01 \\
\mathrm{NR} \\
\mathrm{NR} \\
\mathrm{NE} \\
\mathrm{NE} \\
4.93 \mathrm{E}+01 \\
\mathrm{NE} \\
3.08 \mathrm{E}+02 \\
\mathrm{NE} \\
\mathrm{NR} \\
\mathrm{NE} \\
9.96 \mathrm{E}+01 \\
2.24 \mathrm{E}+02 \\
8.04 \mathrm{E}+01 \\
\mathrm{NE} \\
2.11 \mathrm{E}+00 \\
\mathrm{NE} \\
\mathrm{NE} \\
2.17 \mathrm{E}+00 \\
\mathrm{NR} \\
\mathrm{NE} \\
2.46 \mathrm{E}+00 \\
\mathrm{NE} \\
2.46 \mathrm{E}+00 \\
\mathrm{NE} \\
\mathrm{NE} \\
\end{array}$ & $\begin{array}{c}\text { NR } \\
3.51 \mathrm{E}+01 \\
<1.00 \mathrm{E}-05 \\
8.58 \mathrm{E}-04 \\
\text { NR } \\
1.03 \mathrm{E}-01 \\
7.06 \mathrm{E}-03 \\
\text { NR } \\
8.15 \mathrm{E}+00 \\
\text { NR } \\
\text { NR } \\
3.73 \mathrm{E}-01 \\
\text { NR } \\
<7.81 \mathrm{E}-02 \\
1.18 \mathrm{E}-01 \\
3.34 \mathrm{E}-05 \\
5.61 \mathrm{E}+01 \\
\mathrm{NR} \\
3.08 \mathrm{E}+02 \\
\mathrm{NR} \\
5.10 \mathrm{E}-01 \\
\mathrm{NR} \\
1.09 \mathrm{E}+02 \\
2.36 \mathrm{E}+02 \\
9.49 \mathrm{E}+01 \\
<3.03 \mathrm{E}-01 \\
1.04 \mathrm{E}+01 \\
\mathrm{NR} \\
\mathrm{NR} \\
2.26 \mathrm{E}+00 \\
\mathrm{NR} \\
\mathrm{NR} \\
4.62 \mathrm{E}+00 \\
\mathrm{NR} \\
5.48 \mathrm{E}+00 \\
\mathrm{NR} \\
\mathrm{NR} \\
\end{array}$ & $\begin{array}{c}\text { NE } \\
1.31 \mathrm{E}-02 \\
\mathrm{NE} \\
\mathrm{NE} \\
\mathrm{NE} \\
\mathrm{NE} \\
\mathrm{NE} \\
\mathrm{NE} \\
6.16 \mathrm{E}-02 \\
\mathrm{NE} \\
\mathrm{NE} \\
\mathrm{NR} \\
\mathrm{NR} \\
1.07 \mathrm{E}-02 \\
\mathrm{NE} \\
\mathrm{NE} \\
\mathrm{NR} \\
\mathrm{NE} \\
2.42 \mathrm{E}+00 \\
\mathrm{NE} \\
\mathrm{NR} \\
\mathrm{NE} \\
6.58 \mathrm{E}-01 \\
3.67 \mathrm{E}-01 \\
<2.50 \mathrm{E}-01 \\
\mathrm{NE} \\
5.57 \mathrm{E}-02 \\
\mathrm{NE} \\
\mathrm{NE} \\
1.51 \mathrm{E}-01 \\
\mathrm{NR} \\
\mathrm{NE} \\
4.80 \mathrm{E}-01 \\
\mathrm{NE} \\
7.68 \mathrm{E}-02 \\
\mathrm{NE} \\
\mathrm{NE} \\
\end{array}$ & $\begin{array}{c}\text { NE } \\
1.37 \mathrm{E}-02 \\
\mathrm{NE} \\
\mathrm{NE} \\
\mathrm{NE} \\
\mathrm{NE} \\
\mathrm{NE} \\
\mathrm{NE} \\
9.98 \mathrm{E}-02 \\
\mathrm{NE} \\
\mathrm{NE} \\
\mathrm{NR} \\
\mathrm{NR} \\
1.18 \mathrm{E}-02 \\
\mathrm{NE} \\
\mathrm{NE} \\
\mathrm{NR} \\
\mathrm{NE} \\
2.45 \mathrm{E}+00 \\
\mathrm{NE} \\
\mathrm{NR} \\
\mathrm{NE} \\
9.58 \mathrm{E}-01 \\
5.45 \mathrm{E}-01 \\
2.79 \mathrm{E}-01 \\
\mathrm{NE} \\
6.36 \mathrm{E}-02 \\
\mathrm{NE} \\
\mathrm{NE} \\
1.61 \mathrm{E}-01 \\
\mathrm{NR} \\
\mathrm{NE} \\
4.87 \mathrm{E}-01 \\
\mathrm{NE} \\
8.21 \mathrm{E}-02 \\
\mathrm{NE} \\
\mathrm{NE} \\
\end{array}$ & $\begin{array}{c}\text { NR } \\
1.40 E-02 \\
\text { NR } \\
\text { NR } \\
\text { NR } \\
\text { NR } \\
\text { NR } \\
\text { NR } \\
1.22 E-01 \\
\text { NR } \\
\text { NR } \\
\text { NR } \\
\text { NR } \\
1.43 E-02 \\
<5.50 E-01 \\
\text { NR } \\
\text { NR } \\
\text { NR } \\
2.47 E+00 \\
\text { NR } \\
\text { NR } \\
\text { NR } \\
1.04 E+00 \\
6.14 E-01 \\
3.19 E-01 \\
\text { NR } \\
7.08 E-02 \\
\text { NR } \\
\text { NR } \\
1.69 E-01 \\
\text { NR } \\
\text { NR } \\
5.01 E-01 \\
\text { NR } \\
8.87 E-02 \\
\text { NR } \\
\text { NR }\end{array}$ \\
\hline Radionuclide & $(\mathrm{Bq} / \mathrm{L})$ & $(\mathrm{Bq} / \mathrm{L})$ & $(\mathrm{Bq} / \mathrm{L})$ & - $\quad(\mathrm{Bq} / \mathrm{L})$ & $(\mathrm{Bq} / \mathrm{L})$ & $(\mathrm{Bq} / \mathrm{L})$ \\
\hline $\begin{array}{c}\text { Am-241 } \\
\text { Cs-137 } \\
\text { Np-237 } \\
\text { Pu-238 } \\
\text { Pu-239/240 } \\
\text { Sr-89/90 } \\
\text { Tc-99 }\end{array}$ & $\begin{array}{c}3.45 \mathrm{E}+04 \\
1.12 \mathrm{E}+10 \\
\mathrm{NR} \\
\mathrm{NR} \\
1.65 \mathrm{E}+04 \\
3.42 \mathrm{E}+07 \\
4.74 \mathrm{E}+06\end{array}$ & $\begin{array}{c}4.42 \mathrm{E}+04 \\
1.63 \mathrm{E}+10 \\
\mathrm{NR} \\
\mathrm{NR} \\
4.24 \mathrm{E}+04 \\
3.61 \mathrm{E}+07 \\
5.62 \mathrm{E}+06\end{array}$ & $\begin{array}{c}5.40 E+04 \\
1.71 E+10 \\
<1.13 E+02 \\
N R \\
5.37 E+04 \\
2.83 E+08 \\
1.63 E+07\end{array}$ & $\begin{array}{c}<2.54 \mathrm{E}+03 \\
1.18 \mathrm{E}+08 \\
\mathrm{NR} \\
\mathrm{NR} \\
<4.51 \mathrm{E}+02 \\
7.74 \mathrm{E}+06 \\
\mathrm{NR}\end{array}$ & $\begin{array}{c}2.55 E+04 \\
1.24 E+08 \\
. N R \\
N R \\
9.58 E+02 \\
8.10 E+06 \\
N R\end{array}$ & $\begin{array}{c}7.13 E+04 \\
1.29 E+08 \\
N R \\
N R \\
1.85 E+03 \\
8.54 E+06 \\
\text { NR }\end{array}$ \\
\hline $\begin{array}{c}\text { Physical } \\
\text { Properties }\end{array}$ & Minimum & Best Estimate & Maximum & Minimum & Best Estimate & Maximum \\
\hline $\begin{array}{c}\text { Tank Volume, L* } \\
\text { Density, g/mL } \\
\text { pH } \\
\text { Vol\% solids } \\
\% H 2 O\end{array}$ & $\begin{array}{l}\mathrm{NE} \\
1.5 \\
\mathrm{NR} \\
\mathrm{NE} \\
42\end{array}$ & $\begin{array}{c}4.27 \mathrm{E}+06 \\
1.5 \\
>14 \\
\mathrm{NR} \\
44\end{array}$ & $\begin{array}{l}\mathrm{NE} \\
1.6 \\
>14 \\
\mathrm{NR} \\
45\end{array}$ & $\begin{array}{c}\mathrm{NE} \\
1.0 \\
10.8 \\
\mathrm{NE} \\
98\end{array}$ & $\begin{array}{c}2.98 \mathrm{E}+06 \\
1.0 \\
11.3 \\
\mathrm{NE} \\
99\end{array}$ & $\begin{array}{c}\mathrm{NE} \\
1.0 \\
11.9 \\
\mathrm{NR} \\
99\end{array}$ \\
\hline
\end{tabular}

* Volume based on Hanion 1996 (April, 1996).

NE: Not Estimated

NR: Not Reported in source documents 
Table 2.3. Cont.

\begin{tabular}{|c|c|c|c|c|c|c|}
\hline $\begin{array}{c}\text { Chemical . } \\
\text { Analyte }\end{array}$ & $\begin{array}{c}\text { Minimum } \\
(\mathrm{g} / \mathrm{L})\end{array}$ & $\begin{array}{c}\text { AZ101 } \\
\text { Best Estimate } \\
(\mathrm{g} / \mathrm{L})\end{array}$ & $\begin{array}{c}\text { Maximum } \\
(\mathrm{g} / \mathrm{L})\end{array}$ & $\begin{array}{c}\text { Minimum } \\
(\mathrm{g} / \mathrm{L})\end{array}$ & $\begin{array}{c}\mathrm{AZ} 102 \\
\text { Best Estimate } \\
(\mathrm{g} / \mathrm{L})\end{array}$ & $\begin{array}{l}\text { Maximum } \\
(\mathrm{g} / \mathrm{L})\end{array}$ \\
\hline $\mathrm{Ag}$ & NE & $\mathrm{NE}$ & NR & $N E$ & $N E$ & $6.38 \mathrm{E}-03$ \\
\hline Al & $9.22 \mathrm{E}+\infty 0$ & $9.33 E+00$ & $9.48 \mathrm{E}+00$ & $1.54 \mathrm{E}+00$ & $1.55 E+00$ & $1.56 \mathrm{E}+00$ \\
\hline As & NE & NE & $7.15 \mathrm{E}-03$ & . NE & $N E$ & $4.27 \mathrm{E}-03$ \\
\hline $\mathrm{Ba}$ & NE & NE & $2.55 \mathrm{E}-03$ & NE & NE & $7.83 \mathrm{E}-04$ \\
\hline $\mathrm{Be}$ & NE & NE & $1.12 \mathrm{E}-04$ & NE & $N E$ & $1.13 \mathrm{E}-04$ \\
\hline $\mathrm{Ca}$ & NE & NE & $1.04 \mathrm{E}-02$ & NE & NE & $6.03 \mathrm{E}-03$ \\
\hline $\mathrm{Cd}$ & NE & NE & $4.46 \mathrm{E}-03$ & $\mathrm{NE}$ & NE & $1.92 \mathrm{E}-03$ \\
\hline $\mathrm{Ce}$ & $\mathrm{NE}$ & $\mathrm{NE}$ & NR & NE & $\mathrm{NE}$ & $1.18 \mathrm{E}-02$ \\
\hline $\mathrm{Cl}$ & $1.73 E-01$ & $1.81 E-01$ & 1.87E-01 & $6.83 \mathrm{E}-02$ & $6.83 \mathrm{E}-02$ & $6.83 E-02$ \\
\hline $\mathrm{CN}$ & $\mathrm{NE}$ & NE & NR & NE & $\mathrm{NE}$ & NR \\
\hline Co & NE & NE & NR & NE & $\mathrm{NE}$ & $8.53 E-03$ \\
\hline $\mathrm{Cr}$ & 8.51E-02 & 5.37E-01 & $8.11 \mathrm{E}-01$ & $1.00 \mathrm{E}+00$ & $1.00 \mathrm{E}+00$ & $1.00 \mathrm{E}+00$ \\
\hline Cs & NR & NR & NR & NR & NR & NR \\
\hline$F$ & $1.54 \mathrm{E}+00$ & $1.58 \mathrm{E}+00$ & $1.62 \mathrm{E}+00$ & 7.98E-01 & $1.00 \mathrm{E}+00$ & $1.08 \mathrm{E}+00$ \\
\hline $\mathrm{Fe}$ & NE & NE & $<1.01 \mathrm{E}-02$ & NE & NE & $1.08 \mathrm{E}-02$ \\
\hline $\mathrm{Hg}$ & $\mathrm{NE}$ & NE & NR & NE & NE & NR \\
\hline $\bar{K}$ & $2.99 E+00$ & $4.07 E+00$ & $4.69 \mathrm{E}+00$ & $1.44 \mathrm{E}+00$ & $1.44 \mathrm{E}+00$ & $1.44 \mathrm{E}+00$ \\
\hline $\mathrm{La}$ & $\mathrm{NE}$ & NE & $3.96 \mathrm{E}-03$ & NE & NE & $1.74 \mathrm{E}-03$ \\
\hline $\mathrm{Na}$ & $9.36 \mathrm{E}+01$ & $9.52 \mathrm{E}+01$ & $9.73 E+01$ & $5.29 \mathrm{E}+01$ & $5.32 E+01$ & $5.37 \mathrm{E}+01$ \\
\hline $\mathrm{Nd}$ & NE & NE & $1.43 \mathrm{E}-03$ & NE & NE & $5.42 \mathrm{E}-03$ \\
\hline $\mathrm{NH}_{4}^{+}$ & NR & NR & NR & NR & NR & NR \\
\hline $\mathrm{Ni}$ & NE & $N E$ & - NR & NE & NE & $2.14 \mathrm{E}-03$ \\
\hline $\mathrm{NO}_{2}^{-}$ & $5.51 \mathrm{E}+01$ & $5.68 \mathrm{E}+01$ & $5.90 \mathrm{E}+01$ & $2.22 \mathrm{E}+01$ & $2.76 \mathrm{E}+01$ & $2.96 \mathrm{E}+01$ \\
\hline $\mathrm{NO}_{3}^{-}$ & $6.47 \mathrm{E}+01$ & $6.60 E+01$ & $6.83 E+01$ & $1.91 \mathrm{E}+01$ & $2.37 \mathrm{E}+01$ & $2.59 \mathrm{E}+01$ \\
\hline $\mathrm{OH}$ & $1.12 \mathrm{E}+01$ & $1.13 \mathrm{E}+01$ & $1.14 \mathrm{E}+01$ & $1.83 E+00$ & $1.88 \mathrm{E}+00$ & $1.99 \mathrm{E}+00$ \\
\hline $\mathrm{Pb}$ & NE & $N E$ & $8.29 E-02$ & NE & $N E$ & $5.91 \mathrm{E}-03$ \\
\hline $\mathrm{PO}_{4}^{-3}$ & $1.22 \mathrm{E}+00$ & $1.30 \mathrm{E}+00$ & $1.37 \mathrm{E}+00$ & 1.47E-01 & $1.47 \mathrm{E}-01$ & $1.47 \mathrm{E}-01$ \\
\hline $\mathrm{Sb}$ & NE & NE & NR & $\mathrm{NE}$ & NE & $<1.03 \mathrm{E}-02$ \\
\hline $\mathrm{Se}$ & NE & $\mathrm{NE}$ & $2.84 \mathrm{E}-01$ & NE & NE & $1.08 \mathrm{E}-02$ \\
\hline $\mathrm{SO}_{4}^{-2}$ & $1.51 E+01$ & $1.54 \mathrm{E}+01$ & $1.60 \mathrm{E}+01$ & $1.38 \mathrm{E}+01$ & $1.73 E+01$ & $1.88 \mathrm{E}+01$ \\
\hline $\mathrm{Sr}$ & NR & NR & NR & $2.00 \mathrm{E}-04$ & $2.00 \mathrm{E}-04$ & $2.00 \mathrm{E}-04$ \\
\hline $\mathrm{Te}$ & NE & NE & $2.69 E-03$ & NE & NE & $2.62 E-03$ \\
\hline TIC & $5.66 \mathrm{E}+00$ & $5.78 \mathrm{E}+00$ & $5.99 \mathrm{E}+00$ & $5.37 E+00$ & $5.85 \mathrm{E}+00$ & $6.60 \mathrm{E}+00$ \\
\hline $\mathrm{Tl}$ & NE & NE & NR & NE & - NE & $3.80 \mathrm{E}-02$ \\
\hline TOC & $9.38 \mathrm{E}-01$ & $1.04 \mathrm{E}+00$ & $1.09 \mathrm{E}+00$ & $1.40 \mathrm{E}+00$ & $1.50 \mathrm{E}+00$ & $1.58 \mathrm{E}+00$ \\
\hline $\mathrm{U}$ & NE & NE & $7.14 \mathrm{E}-03$ & NE & NE & $1.60 \mathrm{E}+00$ \\
\hline $\mathrm{V}$ & NE & NE & $8.84 \mathrm{E}-04$ & NE & NE & $2.03 \mathrm{E}-03$ \\
\hline Radionuclide & $(B q / L)$ & $(\mathrm{Bq} / \mathrm{L})$ & $(\mathrm{Bq} / \mathrm{L})$ & $(\mathrm{Bq} / \mathrm{L})$ & $(\mathrm{Bq} / \mathrm{L})$ & $(\mathrm{Bq} / \mathrm{L})$ \\
\hline Am-241 & $\overline{5.86 E+02}$ & $2.44 \mathrm{E}+05$ & $4.87 \mathrm{E}+05$ & NR & $5.44 \mathrm{E}+04$ & NR \\
\hline Cs-137 & $5.86 \mathrm{E}+10$ & $5.87 \mathrm{E}+10$ & $5.88 \mathrm{E}+10$ & $3.81 \mathrm{E}+10$ & $3.86 \mathrm{E}+10$ & $3.92 \mathrm{E}+10$ \\
\hline Np-237 & NR & NR & $<2.62 \mathrm{E}+03$ & NR & $7.04 \mathrm{E}+04$ & NR \\
\hline Pu-238 & NR & NR & $<2.87 E+03$ & NR & $<1.48 \mathrm{E}+05$ & NR \\
\hline $\mathrm{Pu}-239 / 240$ & NR & $1.91 \mathrm{E}+01$ & NR & $\mathrm{NR}$ & $<1.41 \mathrm{E}+06$ & NR \\
\hline $\mathrm{Sr}-89 / 90$ & $4.37 \mathrm{E}+07$ & $4.43 E+07$ & $4.55 E+07$ & $5.40 \mathrm{E}+07$ & $6.63 E+07$ & $8.88 E+07$ \\
\hline Tc-99 & $1.20 \mathrm{E}+07$ & $1.31 \mathrm{E}+07$ & $1.42 \mathrm{E}+07$ & NR & $4.22 \mathrm{E}+06$ & NR \\
\hline $\begin{array}{l}\text { Physical } \\
\text { Properties }\end{array}$ & Minimum & Best Estimate & Maximum & Minimum & Best Estimate & Maximum \\
\hline Tank Volume, L* & NE & $3.36 \mathrm{E}+06$ & NE & NE & $3.57 \mathrm{E}+06$ & NE \\
\hline Density, g/mL & 1.2 & 1.2 & 1.2 & 1.1 & 1.1 & 1.1 \\
\hline $\mathrm{pH}$ & 13.5 & 13.5 & 13.6 & 12.9 & 12.9 & 13.0 \\
\hline Vol\% solids & NE & $\mathrm{NE}$ & NR & $\mathrm{NE}$ & $\mathrm{NE}$ & NR \\
\hline$\% \mathrm{H} 2 \mathrm{O}$ & 74 & 74 & 74 & 83 & 84 & 85 \\
\hline
\end{tabular}

* Volume based on Hanlon 1996 (April, 1996). 


\subsection{AN102}

\subsection{Tank Summary}

Tank AN102 is a double-shelled tank with a total volume of $4091 \mathrm{~m}^{3}$ (1081 kgal). It contains $3755 \mathrm{~m}^{3}$ (992 kgal) of complexed concentrate (CC) waste, and includes $337 \mathrm{~m}^{3}(89 \mathrm{kgal})$ solids (Hanlon 1996). Many TRU elements and strontium are soluble in this waste due to the complexants in solution. This tank is designated as a Concentrated Waste Holding Tank (CWHT); therefore, the volume and composition of the waste are not likely to change in the near future.

\subsection{Reference Summary}

Table 3.1 summarizes the references from which the characterization data for waste in tank AN102 were retrieved. The summary includes the reference number from Kaiser's Master Set data, memo date, the reference citation, sample date and type, whether the data in the reference was accepted for use in the Subset data, and comments about the reference.

\subsection{Waste Volume and Sampling Events}

The waste volume and tank sampling event plot is shown in Figure 3.1. The tank entered service when it received non-complexed waste from tank SY102 in September 1981 continuing until December 1982. The tank then received complexant concentrate from January to October 1983. From November 1983 through June 1984, non-complexed waste was added to AN102. During 1984, the tank received low-level waste from the PUREX process in the first quarter, the contents of the tank (except for a heel of $\sim 1140 \mathrm{~m}^{3} / 30 \mathrm{kgal}$ ) were then transferred to AW102 in the second quarter, and the tank was filled with waste from AW101 in the third quarter. PUREX waste $\left(57 \mathrm{~m}^{3}\right)$ was again added in July 1992. No other transfers have occurred. The waste in the tank does not meet the hydroxide specification, and therefore, caustic addition is planned for: December 1997 through September 1998.

The tank is near capacity and the total volume is progressively decreasing as a result of evaporation. The solids volume is measured intermittently with many years between measurements. The result is a step in the solids plot each time the solids were measured. The solids volume, which was last measured in June 1989 , is projected to be constant, but may be increasing slightly.

\subsection{Master Set}

All of the analytical data from the Kaiser references were included in the Master Set. The evaluators compared the Master Set data entry to the original laboratory report to verify that the numbers and units were transcribed correctly and that the units were converted correctly to the standard $\mu \mathrm{g} / \mathrm{L}$ format. Dates and reference numbers were also checked for accuracy.

\subsection{Subset}

To validate the data from the Master Set, source documents containing the data were 
Table 3.1. Summary of Source Documents Containing Waste Characterization Data for AN102

\begin{tabular}{|c|c|c|c|c|c|c|}
\hline $\begin{array}{c}\text { Kaiser } \\
\text { Reference } \\
\text { Number }\end{array}$ & $\begin{array}{c}\text { Memo } \\
\text { Date }\end{array}$ & Source Document* & $\begin{array}{l}\text { Sample } \\
\text { Date }\end{array}$ & $\begin{array}{c}\text { Sample } \\
\text { Type }\end{array}$ & $\begin{array}{l}\text { Accept/ } \\
\text { Reject }\end{array}$ & Comments \\
\hline AN-102-1 & $2 / 28 / 85$ & $\begin{array}{l}\text { Bratzel DR. 1985. Characterization of Complexant } \\
\text { Concentrate Supernate. Internal memo \# } 65453-85-041 \\
\text { to JN Appel dated } 2 / 28 / 85 \text {, Rockwell Hanford } \\
\text { Operations, Richland, WA. }\end{array}$ & Unavailable & Supernatant & Accept & $\begin{array}{l}\text { Sample \# R3639. No solids, } \\
\text { sampled } 15 \mathrm{ft} \text { from bottom, } \\
\text { analyzed for cations, anions, } \\
\text { TOC, density, and } 8 \text { radionuclides } \\
\text { plus U. }\end{array}$ \\
\hline AN-102-2 & $3 / 14 / 85$ & $\begin{array}{l}\text { Bratzel DR. 1985. Characterization of Complexant } \\
\text { Concentrate Solids from Tank 107-AN, 102-AN, and } \\
101-A Y \text {. Internal memo \# 65453-85-053 to JN Appel } \\
\text { dated 3/14/85, Rockwell Hanford Operations, Richland, } \\
\text { WA. }\end{array}$ & Unavailable & Solids & Reject & $\begin{array}{l}\text { Sample \# R3640. Data on solids, } \\
\text { sampled at the bottom of the tank, } \\
\text { not used because there was no } \\
\text { centrate data. }\end{array}$ \\
\hline AN-102-3 & $7 / 6 / 88$ & $\begin{array}{l}\text { Prignano AL. 1988. Tanks 102-AN and 107-AN } \\
\text { Viscosity and Percent Settled Solids Determination. } \\
\text { Internal memo \# 12221-PCL88-155 to DE Scully and } \\
\text { EC Vogt dated 7/6/88, Westinghouse Hanford } \\
\text { Company, Richland, WA. }\end{array}$ & Unavailable & Slurry & Reject & $\begin{array}{l}\text { Viscosity and } \% \text { settled solids } \\
\text { measurements, not used because it } \\
\text { contained no chemical or } \\
\text { radionuclide data. }\end{array}$ \\
\hline AN-102-4 & $5 / 16 / 90$ & $\begin{array}{l}\text { Hall BW. 1990. Toxic Metals Concentrations of DS } \\
\text { and CC Containing Tanks. DSI to DD Stepnewski } \\
\text { dated 5/15/90. Westinghouse Hanford Company, } \\
\text { Richland, WA. }\end{array}$ & Unavailable & Supernatant & Accept & $\begin{array}{l}\text { Results for } \mathrm{Ba}, \mathrm{Cd}, \mathrm{Cr} \text { and } \mathrm{Pb} \text { in } \\
\text { the supernate. }\end{array}$ \\
\hline AN-102-5 & $12 / 16 / 91$ & $\begin{array}{l}\text { Tingey JM and JD Matheson. 1991. Characterization } \\
\text { of Waste from Double-shelled Tank 102-AN. Letter } \\
\text { report to J Appel, Pacific Northwest Laboratory, } \\
\text { Richland, WA. }\end{array}$ & Unavailable & Supernatant & Accept & $\begin{array}{l}\text { Complete analysis of waste from } \\
\text { AN102, includes cations, anions, } \\
\text { TOC, TIC, and various } \\
\text { radionuclides. Data is for } \\
\text { supernate and solids. Only } \\
\text { supernate values were considered. }\end{array}$ \\
\hline AN-102-6 & $12 / 28 / 94$ & $\begin{array}{l}\text { Herting DL. 1994. Characterization of Supernate } \\
\text { Samples from Tank 102-AN. Internal memo \# 8E110- } \\
\text { PCL94-112 to JM Jones dated 12/28/94, Westinghouse } \\
\text { Hanford Company, Richland, WA. }\end{array}$ & $10 / 21 / 94$ & Supernatant & Accept & $\begin{array}{l}\text { Table } 1 \text { - Analysis of centrifuged } \\
\text { supernate samples for cations, } \\
\text { anions, TOC, TIC, Cs, and Sr. } \\
\text { Comparison with other historical } \\
\text { samples (Table 2). Also includes } \\
\text { mass and charge balance. }\end{array}$ \\
\hline
\end{tabular}

* Many source documents are not cleared for public release. They are cited to provide traceablility to the original data. 


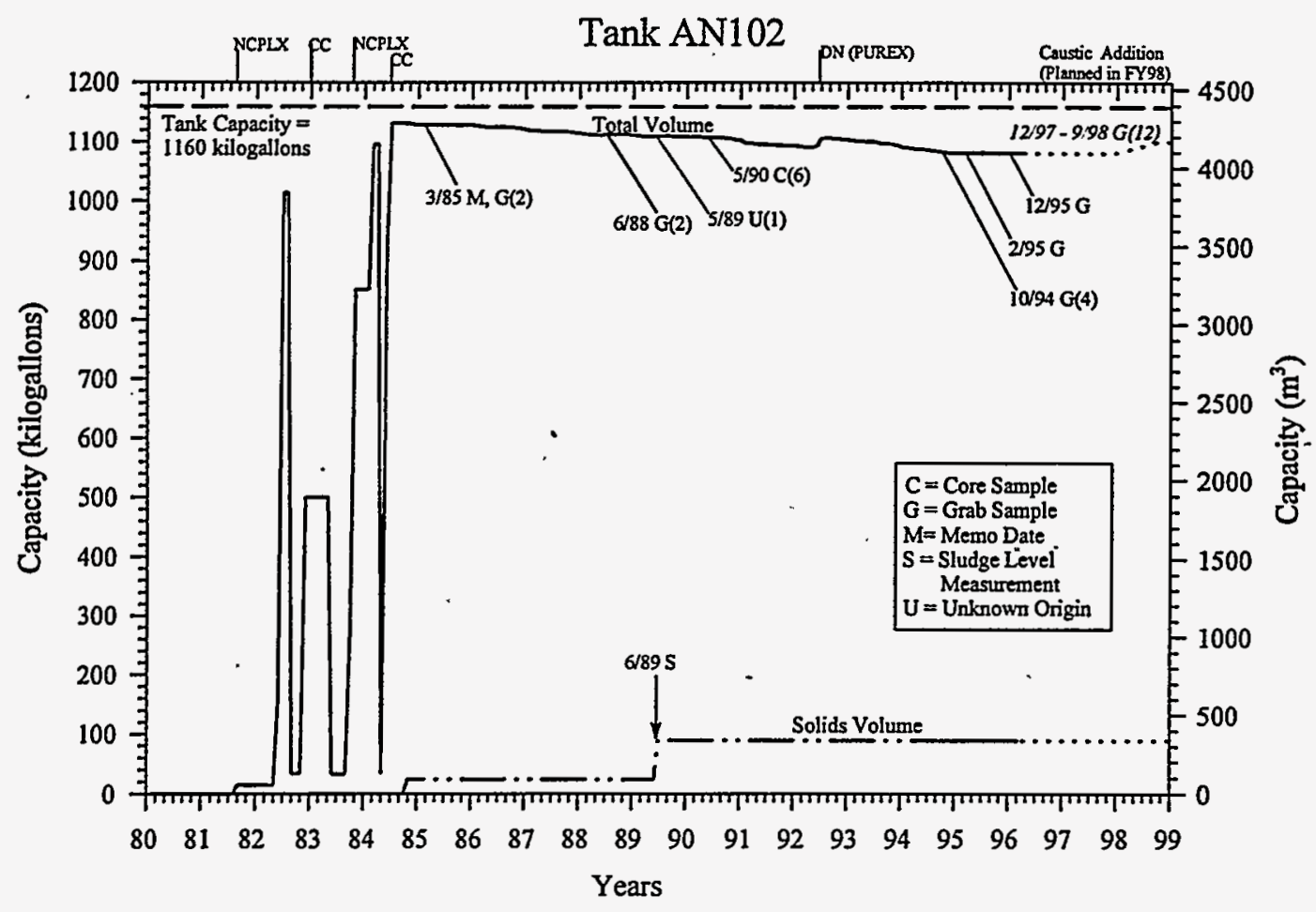

Figure 3.1. Tank Waste Volume and Sampling Event Graph for Tank AN102

reviewed. Of the six source documents that contain chemical and radiochemical data, two contain slurries/solids data only and therefore were not used.

Any TRAC, Braun or Wastren data in the Master Set were not used. The HTCE data were kept but were not factored into the $\mathrm{min} / \mathrm{max} /$ best estimate. Likewise "less than" values were kept but were not used for the $\mathrm{min} / \mathrm{max} / \mathrm{best}$ estimate, unless they were the only data for a species. Duplicate values, such as those listed in a TCR, for a sample were averaged; otherwise different sample aliquots were kept as individual analyses.

The only data outside the Master Set that required validation were the values for total inorganic carbon (TIC) or carbonate $\left(\mathrm{CO}_{3}^{-2}\right)$. The values used were from Kaiser source document number AN-102-6.

\section{6 $\mathrm{Min} / \mathrm{Max} / \mathrm{BE}$}

The lowest and highest concentrations in the Subset were selected as the "Min" and the "Max", respectively. The best estimate was determined by averaging all the valid points in the Subset. 


\subsection{Organics}

No organic speciation measurements have been made on the complexants in tank AN102 waste. However, the complexant species in AN107 waste were measured in 1994 and these measurements can serve as an order of magnitude approximation for the waste in AN102 because the wastes in AN102 and AN107 have similar histories (see section 4.7).

\subsection{Physical Properties}

The visual appearance of the samples varied from opaque coffee-black to translucent brilliant red as described in Kaiser source document AN-102-1. A 1991 sample from a 3-segment core consisted of a dense $(1.51 \mathrm{~g} / \mathrm{mL})$ slurry with $59.7 \mathrm{wt} \%$ solids and a mean particle size of $1.06 \mu \mathrm{m}$. The centrate (centrifuged liquid) from this sample contained $51.6 \mathrm{wt} \%$ solids and had a density of $1.35 \mathrm{~g} / \mathrm{mL}$ (AN-102-5). Other supernate samples were described as very dark brown, nearly black with 1.1-1.3 wt\% solids. The solids contained two layers; the top layer was dark brown and the bottom layer was white. The bottom layer was sodium fluoride diphosphate $\left(\mathrm{Na}_{2} \mathrm{~F}\left(\mathrm{PO}_{4}\right)_{2} \cdot 19 \mathrm{H}_{2} \mathrm{O}\right)$. The dark brown solids were submicron particles that could not be identified (AN-102-6).

\subsection{Limitations/Concerns}

This tank had less analytical data than the other tanks. The data were obtained for specific needs, such as corrosion control, and did not encompass global analyses similar to those for a TCR. Also lacking are organic speciation data, however one could assume that the tank contents were similar to AN107 for which data are available. 


\section{4:0 AN103}

\subsection{Tank Summary}

Tank AN103 has a total volume of $3618 \mathrm{~m}^{3}(956 \mathrm{kgal})$ and contains approximately $3547 \mathrm{~m}^{3}$. (937 kgal) of double-shelled slurry (DSS) (Hanlon 1996). Double-shelled slurry is waste that exceeds the sodium aluminate saturation boundary in the evaporator without exceeding receiver tank composition limits. For reporting purposes, DSS is considered solid waste. Tank AN103 contains waste produced in several evaporator campaigns (the feed from AW, AN, AY, SY, and BX tank farms and other sources). The last major transfer $\left(\sim 2082 \mathrm{~m}^{3} / 550 \mathrm{kgal}\right)$ was from the $86-2$ evaporator campaign in the beginning of 1986. Tank AN103 is designated as a CWHT; therefore, the volume and composition of the waste in this tank are not likely to change in the near future. The tank is on the flammable gas watch list and has been monitored weekly because of the potential for accumulation of hydrogen or flammable gas above the flammability limit. No future transactions are planned before the waste is sent to pretreatment.

\subsection{Reference Summary}

Table 4.1 summarizes the references from which the characterization data for waste in tank AN103 were retrieved. The summary includes the reference number from Kaiser's Master Set data, memo date, the reference citation, sample date and type, whether the data in the reference was accepted for use in the Subset data, and comments about the reference. Transfer data for tank AN103 show that composition data for samples taken before evaporator campaign 86-2 (2/8-16/86) are no longer representative of the tank contents. All of the supernatant data were rejected from the evaluated references. Chemical, radionuclide and physical property data for solids in the tank may be available in relevant documents.

\subsection{Waste Volume and Sampling Events}

The waste volume and tank sampling event plot for AN103 is shown in Figure 4.1. When an ' $M$ ' appears next to a date, it indicates the date of a memo, not the date of a sampling event; no sample date was stated in the memo. Tank AN103 contains DSS, which is classified as a solid (see definition in section 4.1).

The stepped appearance of the solids-volume plot is due to the measuring intervals. Erroneous total solids volumes in 1987 and 1988 caused the reported solids volume to exceed the tank capacity and the total waste volume. The erroneous measurements were not plotted. The solids volume was last measured in August 1989.

Only two core samples were taken after the 86-2 evaporator campaign (in December 1986 or January 1987 and in March or April 1987). Several core segments were missing from the first 
Table 4.1. Summary of Source Documents Containing Waste Characterization Data for AN103

\begin{tabular}{|c|c|c|c|c|c|c|}
\hline $\begin{array}{c}\text { Kaiser } \\
\text { Reference } \\
\text { Number }\end{array}$ & $\begin{array}{c}\text { Memo } \\
\text { Date }\end{array}$ & Source Document* & $\begin{array}{c}\text { Sample } \\
\text { Date }\end{array}$ & $\begin{array}{c}\text { Sample } \\
\text { Type }\end{array}$ & $\begin{array}{l}\text { Accept/ } \\
\text { Reject }\end{array}$ & Comments \\
\hline AN-103-1 & $7 / 2 / 84$ & $\begin{array}{l}\text { Jansky MT. 1984. Laboratory Support for } \\
\text { Tank Farm Samples from Tanks } 103 \text { and } \\
104 \text { AN. Internal letter \# } 65453-84-164 \text { to } \\
\text { PJ Certa dated } 7 / 2 / 84 \text {, Rockwell Hanford } \\
\text { Operations, Richland, WA. }\end{array}$ & Unavailable & & Reject & $\begin{array}{l}\text { - Outdated document (prior to 2/86) } \\
\text { - Sample received from AN103 by Chem. Lab. } \\
\text { Unit in April } 1984 \text { (sample \# R9990) } \\
\text { - Analyses of centrifuged supernatant and solids } \\
\text { - Limited analytical details } \\
\text { - Similar nitrite and nitrate concentration in } \\
\text { solids for AN103 and AN104 but significant } \\
\text { Am-241 in AN104 and none in AN103 }\end{array}$ \\
\hline AN-103-2 & $1 / 18 / 85$ & $\begin{array}{l}\text { Mauss BM and MT Jansky. 1985. 102- } \\
\text { AW Laboratory Boildowns in Support of } \\
\text { Evaporator Run } 84-5 \text {. Internal memo \# } \\
65453-85-013 \text { to EG Gratny and NL } \\
\text { Pontious dated 1/18/85, Rockwell Hanford } \\
\text { Operations, Richland, WA. }\end{array}$ & Unavailable & . & Reject & $\begin{array}{l}\text { - Outdated document (prior to 2/86) } \\
\text { - 242-A Evaporator Campaign Run 84-3 Doc } \\
\text { - DSSF product (425") from evaporator was } \\
\text { stored in AN103 and AN104 } \\
\text { - Chemical composition table (p 77) for AN103 } \\
\text { sample \# T8882 included without supporting } \\
\text { documentation }\end{array}$ \\
\hline$A N-103-3$ & $1 / 25 / 85$ & $\begin{array}{l}\text { Mauss BM. 1985. Laboratory Support of } \\
242-A \text { Evaporator Run 85-2. Internal } \\
\text { memo \# 65453-85-018 to NL Pontious } \\
\text { dated } 1 / 25 / 85 \text {, Rockwell Hanford } \\
\text { Operations, Richland, WA. }\end{array}$ & Unavailable & see note & Reject & $\begin{array}{l}\text { - Outdated document (prior to 2/86) } \\
\text { 242-A Evaporator Campaign Run 85-2 Doc. } \\
\text { feed (AN104 and AN105) } \\
\text { - "dip" samples, a bottom sample(R-3644) and } \\
\text { a top sample (R-3645) } \\
\end{array}$ \\
\hline AN-103-4 & $12 / 9 / 92$ & $\begin{array}{l}\text { Certa PJ. 1992. 242-A Evaporator/ } \\
\text { Crystallizer FY 84 Campaign Run 84-3 Post } \\
\text { Run Document. WHC-SD-WM-PE-018, } \\
\text { Westinghouse Hanford Company, Richland, } \\
\text { WA. }\end{array}$ & Unavailable & & Reject & $\begin{array}{l}\text { - Outdated document (prior to } 2 / 86 \text { ) } \\
\text { - 242-A Evaporator Campaign Run 84-3 Doc } \\
\text { - DSSF product (425") from evaporator was } \\
\text { stored in AN103 and AN104 } \\
\text { - Chemical composition table (p 77) for AN103 } \\
\text { sample \# T8882 included without supporting } \\
\text { documentation }\end{array}$ \\
\hline AN-103-5 & $3 / 28 / 86$ & $\begin{array}{l}\text { Mauss BM. 1986. The 86-2 Evaporator } \\
\text { Campaign: Laboratory Results on the } \\
\text { Production of Double-shelled Slurry. } \\
\text { Internal letter \#65453-86-038 to MG Kelly } \\
\text { dated } 3 / 28 / 86 \text {, Rockwell Hanford } \\
\text { Operations, Richland, WA. }\end{array}$ & $2 / 86$ & $\begin{array}{l}\text { Sludge } \\
\text { Slurry } \\
\text { (product of } \\
\text { the } \\
\text { campaign) }\end{array}$ & Reject & $\begin{array}{l}\text { Composition of sample \# R-8190 and } \\
\text { evaporator products may be used to roughly } \\
\text { estimate the liquid composition in the tank, } \\
\text { but would need additional information about } \\
\text { actual transfers during campaign. } \\
\text { - Lab results on the production of DSS }\end{array}$ \\
\hline
\end{tabular}




\begin{tabular}{|c|c|c|c|c|c|c|}
\hline $\begin{array}{l}\text { Kaiser } \\
\text { Reference } \\
\text { Number }\end{array}$ & $\begin{array}{c}\text { Memo } \\
\text { Date }\end{array}$ & Source Document* & $\begin{array}{c}\text { Sample } \\
\text { Date }\end{array}$ & $\begin{array}{c}\text { Sample } \\
\text { Type }\end{array}$ & $\begin{array}{l}\text { Accept/ } \\
\text { Reject }\end{array}$ & Comments \\
\hline AN-103-6 & $7 / 87$ & $\begin{array}{l}\text { Fow CL. 1987. Characterization of Waste } \\
\text { From Double-shelled Tank 103-AN. Letter } \\
\text { Report \# 7W21-87-14, Pacific Northwest } \\
\text { Laboratory, Richland, WA. }\end{array}$ & Unavailable & $\begin{array}{c}\text { Core } \\
\text { sample } \\
\text { (segments } \\
9,12 \text {, and } \\
16 \text { ) }\end{array}$ & Reject & $\begin{array}{l}\text { Supernate data were not available. } \\
\text { - Characterization of waste from DST AN103 } \\
\text { (Physical properties of core samples) }\end{array}$ \\
\hline AN-103-7 & $1 / 13 / 88$ & $\begin{array}{l}\text { Prignano AL. 1988. Dissolution Study of } \\
\text { Composite from Tank 103-AN. Internal } \\
\text { Memo \# 1221-PCL88-060 to DR Bratzel et } \\
\text { al. dated 1/13/88, Westinghouse Hanford } \\
\text { Company, Richland, WA. }\end{array}$ & $\cdot$ & $\begin{array}{l}\text { Core } \\
\text { sample }\end{array}$ & Reject & $\begin{array}{l}\text { The document contained limited chemical } \\
\text { analyses of supernate, but many problems } \\
\text { were found with the data. } \\
\text { Dissolution Study of Composite from Tank } \\
\text { AN103 }\end{array}$ \\
\hline AN-103-8 & $5 / 16 / 90$ & Report Analysis Data Sheet dated $5 / 16 / 90$ & Unavailable & Sludge & Reject & $\begin{array}{l}\text { - Supernate data were not available. } \\
\text { - Data sheet lists } 8 \text { toxic metals concentrations } \\
\text { in sludge with limited additional information. }\end{array}$ \\
\hline AN-103-9 & $9 / 7 / 90$ & Report Analysis Data Sheet dated $9 / 7 / 90$ & Unavailable & $\begin{array}{c}\text { Core } \\
\text { sample }\end{array}$ & Reject & $\begin{array}{l}\text { - Supernate data were not available. } \\
\text { Data sheet lists slurry sample inorganic and } \\
\text { radionuclide concentrations with limited } \\
\text { analytical information. }\end{array}$ \\
\hline
\end{tabular}




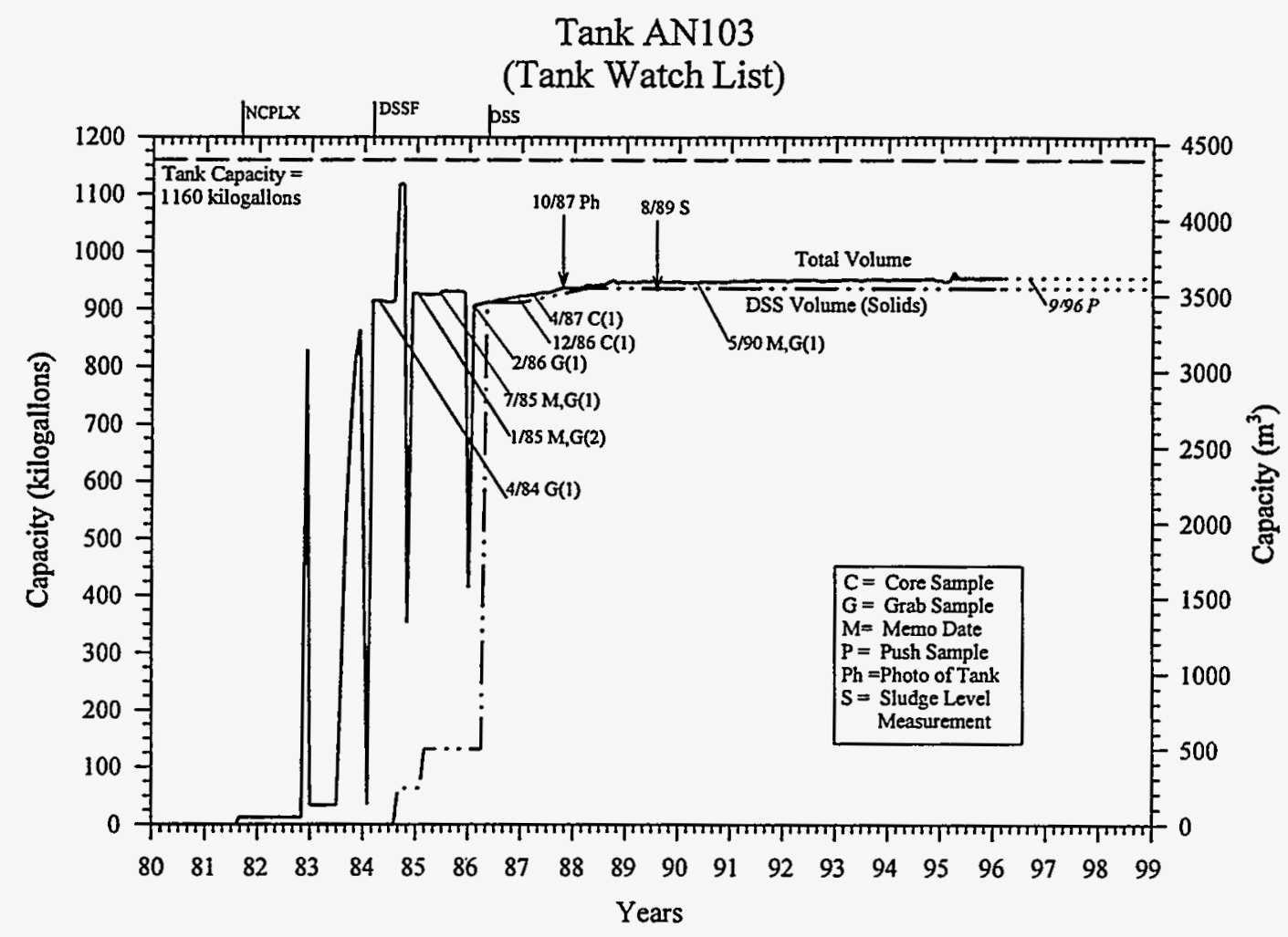

Figure 4.1. Tank Waste Volume and Sampling Event Graph for Tank AN103

sample, and resampling was done to obtain those segments. The discrepancy in the core sampling dates arises from differences in the letters and reported the dates: 12/86 (a), $1 / 87$ (AN-103-6), 3/87 (AN-103-6), and 4/87 (core sampling summary sheet).

\subsection{Master Set/Subset}

All of the analytical data acquired by Kaiser were included in the Master Set. However, all original source documents were rejected because they either preceded an evaporator campaign or contained no valid supernatant data. Therefore, no Subset was created and no minimum, maximum, and best estimate values were generated.

\subsection{Physical Properties}

Physical property measurements were performed on core samples retrieved from AN103 in January 1987 (AN-103-6). The properties measured were density, volume percent centrifuged

(a) Mudge KL. 1987. Core Sample Results 103-AN. Internal memo \# 65950-87-019 to KW Owens dated 1/12/87, Rockwell Hanford Operations, Richland, WA. 
solids, volume percent settled solids, weight percent total solids, and the rheological properties of viscosity and shear strength. The measurements were intended to provide information for pumping the slurry in AN103 to the AP tank farm. However, this transfer has not occurred.

\subsection{Limitations/Concerns}

The last major transfer ( $\left.2082 \mathrm{~m}^{3} / 550 \mathrm{kgal}\right)$ followed the $86-2$ evaporator campaign in the beginning of 1986 (AN-103-5, AN-103-6). The waste type designation, however, was not changed from Double-shelled Slurry Feed (DSSF) to DSS until May 1986. Goheen reported a different last transfer date, at the end of 1985 (See appendix B). Total solids volume reported in 1987 and 1988 exceeded the tank capacity and the total waste volume. In the same report, the current status summary incorrectly reports the value for the total waste volume as the supernatant value. Other contradictory data were found in Waste Tank Summary Reports (WHC-EP-0182-82, -85, and -87), which gives two values for solids volume ( $373 \mathrm{kgal}$ solids in Table B-2 and $937 \mathrm{kgal}$ DSS in Table E-5 of the Waste Tank Summary Report).

Analytical data for the tank composition of AN103 were reported in a WHC internal memo. No sampling information was available in this report, but an earlier report ${ }^{(\mathrm{a})}$ referred to dissolution studies that would be performed on the $12 / 86$ (or 1/87) and $3 / 87$ (or 4/87) core samples. Core segments were used to prepare composite samples for physical, rheological, chemical, radiological and dissolution analyses. A limited chemical analysis $\left(\mathrm{Cl}, \mathrm{CO}_{3}^{-2}, \mathrm{TOC}, \mathrm{F}\right.$, $\mathrm{NO}_{2}^{-}, \mathrm{NO}_{3}^{-}, \mathrm{OH}, \mathrm{PO}_{4}^{-3}, \mathrm{SO}_{4}^{-2}, \mathrm{Na}$, and $\mathrm{Al}$ ) of $\mathrm{AN} 103$ supernate was reported in the WHC internal memo (AN-103-7). However, this source is a poor representation of the tank composition, as demonstrated by the following list:

1. The solubility tests were run at $10^{\circ} \mathrm{C}$. This temperature is probably lower than the actual tank temperature, and data may not be representative of the actual tank slurry.

2. The densities reported for the supernate $(0.80 \mathrm{~g} / \mathrm{mL}$ and $1.04 \mathrm{~g} / \mathrm{mL})$ seem unreasonable for a slurry composed primarily of sodium nitrate $\left(\mathrm{NaNO}_{3}\right)$. The expected density of a concentrated sodium nitrate solution would be $\sim 1.2 \mathrm{~g} / \mathrm{mL}$.

3. The units (moles dissolved/gram slurry) reported for the supernate baseline samples seem to be in error. The following conversion to moles of analyte in supernatant per liter of supernatant for core number 14 uses the $\mathrm{NO}_{3}^{-}$concentration in the supernate $(0.007 \mathrm{~mol} / \mathrm{g}$ slurry) and the density of the slurry $(1.5-1.8 \mathrm{~g} / \mathrm{mL}$ ). (Another conversion can be performed using the supernate density of $1.04 \mathrm{~g} / \mathrm{mL}$ and the weight fraction of supernate in slurry.)

$$
\frac{0.007 \mathrm{~mol} \mathrm{NO}_{3-}^{-}}{\mathrm{g} \text { slurry }} \times \frac{1.7 \mathrm{~g} \text { slurry }}{\mathrm{mL} \text { slurry }} \times \frac{3 \mathrm{~mL} \text { slurry }}{0.45 \mathrm{~mL} \text { sup }} \times \frac{1000 \mathrm{~mL} \text { sup }}{\mathrm{L} \text { sup }}=\frac{80 \mathrm{~mol} \mathrm{NO}_{3}^{-}}{\mathrm{L} \text { sup }}
$$

The nitrate concentration calculated is unreasonable.

4. The aluminum concentration in the results section of the memo ( 0.07 moles/gram slurry) is

(a) Prignano, AL. 1987. Dissolution Properties of Composite from Tank 103-AN. SD-RE-TP016. Westinghouse Hanford Company, Richland, WA. 
different from that reported for the same sample in the data table $(0.006$ moles/gram slurry).

5. Duplicate data are not available for all analyses.

6. In the dissolution study experiments, the concentration of aluminum reported in the supernatant $(0.3 \mathrm{~mol} / \mathrm{g}$ slurry $)$ is greater than the total amount found in the slurry $(0.07$ $\mathrm{mol} / \mathrm{g}$ slurry). Also, $0.3 \mathrm{~mol}$ of aluminum is equivalent to $8 \mathrm{~g}$ of aluminum, which cannot be present in $1 \mathrm{~g}$ of slurry. (It is likely that units should be $\mathrm{g} / \mathrm{g}$ slurry.)

Therefore, these data are highly questionable and were not used for the Subset database.

An estimate of the liquid composition for AN103, may be possible from interstitial liquid from a sludge sample (R-8190, sampled during 86-2 evaporator campaign) and the 86-2 evaporator products (R-8118, 8119, and 8120 , which were representative of the product being transferred to AN103) (AN-103-5). Additional information regarding the volumes of evaporator product transferred to AN103 when these sampling events occurred is needed. 


\section{5:0 AN104}

\subsection{Tank Summary}

Tank AN104 contains 4012 (1060 kgal) of DSSF waste (Hanlon 1996). The waste volume is $3013 \mathrm{~m}^{3}$ (796 kgal) of supernatant and $1000 \mathrm{~m}^{3}$ (264 kgal) of sludge. Currently, this tank is designated by WHC as a CWHT, which means that the volume and composition of the waste in this tank are not likely to change in the near future. The waste in this tank has been on the hydrogen watch list since January 1991 and, therefore, the tank is actively ventilated. Temperature is monitored in compliance with the operations safety document, which specifies DST temperature limits, gradients, etc (WHC 1996).

\subsection{Reference Summary}

Table 5.1 summarizes the references from which the characterization data for waste in tank AN104 were retrieved. The summary includes the reference number from Kaiser's Master Set data, memo date, the reference citation, sample date and type, whether the data in the reference was accepted for use in the Subset data, and comments about the reference. Based on tank transfer data for tank AN104, tank composition data from samples taken before the second quarter of 1985 no longer represent the current contents of the tank. All of the references included for assessment refer to data collected before that date and are therefore irrelevant. Source document AN-104-6 was issued in 1990, but it refers to a sample taken before the second quarter of 1985 .

\subsection{Waste Volume and Sampling Events}

The waste volume and tank sampling event plot for AN104 is shown in Figure 5.1. The waste has not been sampled since the last transfer, which was in October 1985. According to the Hanlon report (1995), the most recent tank level volume changes for AN104 were in the fourth quarter of 1984 and the second quarter of 1985 . These volume changes are reported approximately six months later than the dates of evaporator campaigns for this tank (Run 84-3 from 12/83 to 3/84 and Run 84-5 from 5/84 to 10/84) (AN-104-3, AN-104-5b). The stepped appearance of the solids volume plot reflects the measuring intervals. The solids volume, which was last measured in August 1989, is projected to be constant but may be increasing slightly. No future transactions are planned before the waste is sent to pretreatment.

\subsection{Master Set/Subset}

All of the analytical data acquired by Kaiser were included in the Master Set. However, all original source documents were rejected because they preceded an evaporator campaign. Therefore no Subset was created, and no minimum, maximum, and best estimate values were generated.

\subsection{Limitations/Concerns}

Tank volume and sample history data show that the last two transfers occurred in fourth 
Table 5.1. Summary of Source Documents Containing Waste Characterization Data for AN104

\begin{tabular}{|c|c|c|c|c|c|c|}
\hline $\begin{array}{l}\text { Kaiser } \\
\text { Reference } \\
\text { Number }\end{array}$ & $\begin{array}{c}\text { Memo } \\
\text { Date }\end{array}$ & Source Document* & $\begin{array}{l}\text { Sample } \\
\text { Date }\end{array}$ & $\begin{array}{c}\text { Sample } \\
\text { Type }\end{array}$ & $\begin{array}{l}\text { Accept/ } \\
\text { Reject }\end{array}$ & Comments \\
\hline AN-104-1 & $4 / 2 / 84$ & $\begin{array}{l}\text { Jansky MT. 1984. Laboratory Batch } \\
\text { Boildown on Actual Double Shell Slurry } \\
\text { Feed: AN104 and AN105 Blend. } \\
\text { Internal Letter \# 65453-84-090 to LM } \\
\text { Sasaki dated 4/2/84, Rockwell Hanford } \\
\text { Operations, Richland, WA. }\end{array}$ & Unavailable & $\begin{array}{l}\text { Blended } \\
\text { Slurry }\end{array}$ & Reject & $\begin{array}{l}\text { Outdated document (prior to Q2 1985). Analytical } \\
\text { results for blended DSSF samples (AN104 and } \\
\text { AN105) from waste volume reduction experiments. } \\
\text { Experiments performed to examine slurry growth of } \\
\text { AN104 and AN105 blended samples. }\end{array}$ \\
\hline AN-104-2a & $4 / 5 / 84$ & $\begin{array}{l}\text { Sasaki LM. 1984. Results of } \\
\text { Organic Analysis of Double Shell } \\
\text { Slurry. Internal Letter \# } 65611-84-053 \\
\text { to DJ Fisher dated } 4 / 5 / 84, \text { Rockwell } \\
\text { Hanford Operations, Richland, WA. }\end{array}$ & Unavailable & $\begin{array}{l}\text { Blended } \\
\text { Slurry }\end{array}$ & Reject & $\begin{array}{l}\text { Outdated document (prior to Q2 1985). Results of } \\
\text { organic analysis on DSS (blended) samples as part of } \\
\text { slurry growth studies. Initial DSS boildown } \\
\text { composition from reference \#AN-104-1. }\end{array}$ \\
\hline AN-104-2b & NA & $\begin{array}{l}\text { Certa PJ. 1985. 242-A Evaporator! } \\
\text { Crystallizer FY 84 Campaign Run } 84-3 \\
\text { Post Run Document. RI-SD-PE-018, } \\
\text { Rev. 0, Rockwell Hanford Operations, } \\
\text { Richland, Washington. } \\
\end{array}$ & Unavailable & $\begin{array}{l}\text { Supernatant } \\
\text { / Solids }\end{array}$ & Reject & $\begin{array}{l}\text { Outdated document (prior to Q2 1985). 242-A } \\
\text { Evaporator FY84 Campaign Run } 84-3 \text { processed } \\
\text { dilute DSSF from 12/83-3/84. DSSF product ( } 425 \\
\text { in or } 1170 \mathrm{kgal} \text { ) from evaporator was stored in both } \\
\text { AN103 and AN104. Chemical composition table ( } \mathrm{p} \\
\text { 71) for AN104 sample \# R9991 included. See AN- } \\
\text { 104-3 for analytical details. }\end{array}$ \\
\hline AN-104-2c & $6 / 12 / 90$ & $\begin{array}{l}\text { Harris JP. 1990. Tank 104-AN Level } \\
\text { Fluctuations Due to Slurry Growth. } \\
\text { Internal Memo \# } 82331-90-259 \text { to DG } \\
\text { Baide dated 6/12/90, Westinghouse } \\
\text { Hanford Company, Richland, WA. }\end{array}$ & Unavailable & Slurry & Reject & $\begin{array}{l}\text { Irrelevant document, (no analytical data). } \\
\text { Discussions of tank level fluctuations due to slurry } \\
\text { growth. }\end{array}$ \\
\hline AN-104-3 & $7 / 2 / 84$ & $\begin{array}{l}\text { Jansky MT. 1984. Laboratory } \\
\text { Support for Tank Farm Samples from } \\
\text { Tanks } 103 \text { and 104-AN. Internal memo } \\
\text { \# 65453-84-164 to PJ Certa dated } \\
\text { 7/2/84, Rockwell Hanford Operations, } \\
\text { Richland, WA. }\end{array}$ & Unavailable & $\begin{array}{l}\text { Supernatant } \\
\text { / Solids }\end{array}$ & Reject & $\begin{array}{l}\text { Outdated document (prior to Q2 1985). Lab support } \\
\text { for tank farm samples. (AN104 and AN103). Sample } \\
\text { received from AN104 by Chem. Lab. Unit in April, } \\
1984 \text { (sample \# R9991). Sample stored for two } \\
\text { weeks at } 40^{\circ} \mathrm{C} \text {. Lab analyses performed on } \\
\text { supernatant and solids from centrifuged sample. }\end{array}$ \\
\hline
\end{tabular}




\begin{tabular}{|c|c|c|c|c|c|c|}
\hline $\begin{array}{l}\text { Kaiser } \\
\text { Reference } \\
\text { Number }\end{array}$ & $\begin{array}{c}\text { Memo } \\
\text { Date }\end{array}$ & Source Document* & $\begin{array}{c}\text { Sample } \\
\text { Date }\end{array}$ & $\begin{array}{c}\text { Sample } \\
\text { Type }\end{array}$ & $\begin{array}{c}\text { Accept/ } \\
\text { Reject }\end{array}$ & Comments \\
\hline $\begin{array}{c}\text { TO-040- } \\
560\end{array}$ & $7 / 26 / 84$ & $\begin{array}{l}\text { Tank Farm Surveillance and Operations } \\
\text { Department. 1988. Tank Farm Sludge } \\
\text { Level Readings. Standard Operating } \\
\text { Procedure TO-040-560, Rockwell } \\
\text { Hanford Operations, Richland, WA. }\end{array}$ & Unavailable & NA & Reject & $\begin{array}{l}\text { Irrelevant document (no analytical data). Data sheet } \\
\text { (pg 19) lists sludge level readings without } \\
\text { accompanying documentation. }\end{array}$ \\
\hline AN-104-4a & $1 / 15 / 85$ & $\begin{array}{l}\text { Mauss BM. 1985. 242-A Evaporator } \\
\text { Run 84-5: Chemical Analysis of Feed } \\
\text { and Slurry Sets. Internal memo \# } \\
65453-85-009 \text { to EG Gratny and NL } \\
\text { Pontious dated 1/15/85, Rockwell } \\
\text { Hanford Operations, Richland, WA. }\end{array}$ & Unavailable & $\begin{array}{l}\text { Slurry } \\
\text { (Filtrate/ } \\
\text { Solids) }\end{array}$ & Reject & $\begin{array}{l}\text { Outdated document,(prior to Q2 1985). Chemical } \\
\text { analyses of feed/slurry sample sets from } 242-A \\
\text { Evaporator Campaign Run } 84-5 \text {. Refer to SD-WM- } \\
\text { PE-022, Rev. } 0 \text { for evaporator run } 84-5 \text { details. } \\
\text { Physical properties, analytes concentrations, } \\
\text { radionuclides for slurry sample \#s: R-3305, R-3307, } \\
\text { R-3413, R-3415. Referenced letter contains } \\
\text { previously reported data set. }\end{array}$ \\
\hline AN-104-4b & $11 / 9 / 84$ & $\begin{array}{l}\text { Mauss BM. 1984. Chemical } \\
\text { Compositions of 102-AY, I01-AW, 105- } \\
A N \text {, and 104- } A W \text {. Internal memo \# } \\
65453-84-348 \text { to EG Gratny dated } \\
11 / 9 / 84 \text {, Rockwell Hanford Operations, } \\
\text { Richland, WA. }\end{array}$ & Unavailable & NA & Reject & $\begin{array}{l}\text { Irrelevant document, (no data for AN104). Error in } \\
\text { document title. Document contained data for AW104 } \\
\text { not AN104. }\end{array}$ \\
\hline $\begin{array}{l}\text { SD-WM- } \\
\text { PE-022, } \\
\text { Rev. } 0\end{array}$ & NA & $\begin{array}{l}\text { Gratny EG. 1985. 242A Evaporator } \\
\text { Crystallizer FY1984 Campaign 84-5 } \\
\text { Post Run Document. SD-WM-PE-022, } \\
\text { Westinghouse Hanford Company, } \\
\text { Richland, WA. }\end{array}$ & Unavailable & NA & Reject & $\begin{array}{l}\text { Outdated document (report compiles evap. run } \\
\text { documents). 242-A Evaporator Campaign Run } 84-5 \\
\text { concentrated non-complexed waste from 5/84-10/84. } \\
\text { Slurry from run sent to AN104. }\end{array}$ \\
\hline AN-104-5a & $1 / 18 / 85$ & $\begin{array}{l}\text { Mauss BM and MT Jansky. } 1985 . \\
\text { l02-AW Laboratory Boildowns in } \\
\text { Support of Evaporator Run } 84-5 \\
\text { Internal memo \# } 65453-85-013 \text { to EG } \\
\text { Gratny and NL Pontious dated } 1 / 18 / 85 \text {, } \\
\text { Rockwell Hanford Operations, } \\
\text { Richland, WA. }\end{array}$ & $\begin{array}{c}\text { Unavailable } \\
.\end{array}$ & Supernatant & Reject & $\begin{array}{l}\text { Outdated document, (prior to Q2 1985). AW106 } \\
\text { and AN104 Lab boildown support of Evap, Run 84- } \\
5 \text {. Analytical results of AN-104 (sample \# R-3184, } \\
\text { Table XIII). }\end{array}$ \\
\hline
\end{tabular}




\begin{tabular}{|c|c|c|c|c|c|c|}
\hline $\begin{array}{c}\text { Kaiser } \\
\text { Reference } \\
\text { Number }\end{array}$ & $\begin{array}{c}\text { Memo } \\
\text { Date }\end{array}$ & Source Document* & $\begin{array}{c}\text { Sample } \\
\text { Date }\end{array}$ & $\begin{array}{c}\text { Sample } \\
\text { Type }\end{array}$ & $\begin{array}{c}\text { Accept/ } \\
\text { Reject }\end{array}$ & Comments \\
\hline AN-104-5b & $1 / 22 / 85$ & $\begin{array}{l}\text { Kelly MG. } 1985 \text {. Samples from Tank } \\
I 04-A N \text {. Internal memo \# } 65611-85- \\
011 \text { to BM Mauss dated } 1 / 22 / 85 \text {, } \\
\text { Rockwell Hanford Operations, } \\
\text { Richland, WA. }\end{array}$ & $1-18-85$ & Supernatant & Reject & $\begin{array}{l}\text { Outdated document, (no analytical data available). } \\
\text { Request for analyses on samples taken from tank } \\
\text { AN104 on 1-18-85 (sample \#s: R4101, R4102). } \\
\text { Data for requested analyses was not located. }\end{array}$ \\
\hline AN-104-6 & $3 / 27 / 90$ & $\begin{array}{l}\text { Harris JP. } 1990 \text {. Curie content of } \\
\text { Tanks AN103, AN104, and AN105. } \\
\text { Internal Memo } \# 82331-90-136 \text { to } \\
\text { Dunford GL dated } 3 / 27 / 90 \text {, } \\
\text { Westinghouse Hanford Company, } \\
\text { Richland, WA. }\end{array}$ & Unavailable & slurry & Reject & $\begin{array}{l}\text { Outdated document, (data reported earlier). Curie } \\
\text { content of tanks AN103, AN104 (sample R-3184), } \\
\text { and AN105. }\end{array}$ \\
\hline
\end{tabular}

* Many source documents are not cleared for public release. They are cited to provide traceablility to the original data. 


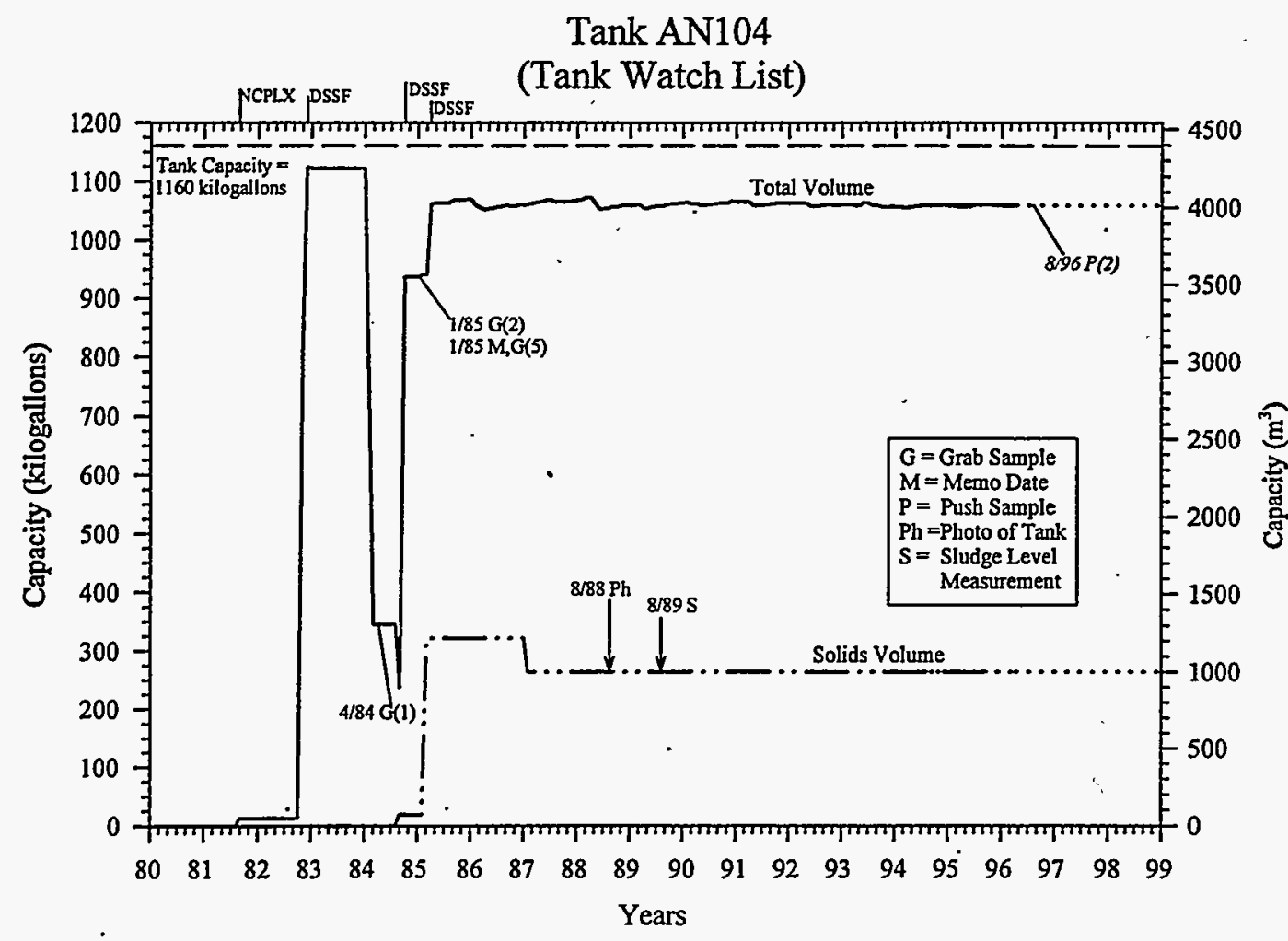

Figure 5.1. Tank Waste Volume and Sampling Event Graph for Tank AN104

quarter of 1984 and second quarter of 1985 . These transfers, which represent $75 \%$ of the waste volume in the tank ( $-3028 \mathrm{~m}^{3} / 800 \mathrm{kgal}$ ) (see appendix B), appear to correspond to evaporator runs that occurred about 6 months earlier. The 84-3 evaporator campaign occurred in 12/83 through 3/84 (AN-104-2b), and evaporator campaign 84-5 occurred in 5/84 through 10/84 (Gratny 1992). The waste transfer dates significantly affect the waste characterization for this tank. Although it is believed that the two sampling events preceded the last evaporator campaign, ${ }^{\text {(a) }}$. the sampling events must be verified against tank volume history data. There are no defensible characterization data for AN104.

(a) On 4/84, a sample was received by the Chem. Lab Unit (AN-104-4a) and support experiments were performed on samples for evaporator run 84-5 (AN-104-3). 


\section{6:0 AN105}

\subsection{Tank Summary}

Tank AN105 contains $4281 \mathrm{~m}^{3}$ (1,131 kgal) of DSSF waste (Hanlon 1996). Double-shelled slurry feed is waste that was concentrated by evaporation just to the point of reaching the saturation level of sodium aluminate. The waste volume is reported to be entirely supernatant. Currently, this tank is designated by WHC as a CWHT, which means that the volume and composition of the waste in this tank are not likely to change in the near future. The tank is on the flammable gas watch list and is monitored weekly because of the potential for hydrogen or flammable gas accumulation above the flammability limit. The last transfer to this tank, in January 1985 was about $3785 \mathrm{~m}^{3}$ (1000 kgal) of waste from AW102 (see appendix B).

\subsection{Reference Summary}

Table 6.1 summarizes the references from which the characterization data for waste in tank AN105 were retrieved. The summary includes the reference number from Kaiser's Master Set data, memo date, the reference citation, sample date and type, whether the data in the reference were accepted for use in the Subset data, and comments about the reference. Tank waste transfer data for AN105 show that waste composition data for samples taken before the second quarter of 1985 no longer represent the contents of the tank. Therefore, all of the references included for assessment of the tank are irrelevant.

\subsection{Waste Volume and Sampling Events}

The waste volume and tank sampling event plot for AN105 is shown in Figure 6.1. The figure shows that tank AN105 is near capacity. The waste in the tank has not been sampled since the last transfer into this tank in January 1985. Measurements of solids volume in October 1984 and an in-tank photograph taken in January 1988 revealed no measurable solids in this tank. No future transactions are planned for this tank before the waste is sent to pretreatment.

\subsection{Master Set/Subset}

All of the analytical data acquired by Kaiser were included in the Master Set. However, all original source documents were rejected because they preceded an evaporator campaign. Therefore no Subset was created, and no minimum, maximum, and best estimate values were generated.

\subsection{Limitations/Concerns}

All of the limited analytical results available for AN105 waste were from sampling events that preceded a major transfer, in which $>90 \%$ of the tank volume was completely drained and replaced with $\sim 3785 \mathrm{~m}^{3}$ (1000 $\left.\mathrm{kgal}\right)$. Thus, the tank waste composition changed dramatically, and the analytical data were no longer representative of the waste composition. 
Table 6.1. Summary of Source Documents Containing Waste Characterization Data for AN105

\begin{tabular}{|c|c|c|c|c|c|c|}
\hline $\begin{array}{c}\text { Kaiser } \\
\text { Reference } \\
\text { Number }\end{array}$ & $\begin{array}{c}\text { Memo } \\
\text { Date }\end{array}$ & Source Document* & $\begin{array}{c}\text { Sample } \\
\text { Date }\end{array}$ & $\begin{array}{c}\text { Sample } \\
\text { Type }\end{array}$ & $\begin{array}{l}\text { Accept/ } \\
\text { Reject }\end{array}$ & Comments \\
\hline AN-105-1 & $4 / 5 / 84$ & $\begin{array}{l}\text { Sasaki LM. 1984. Results of Organic } \\
\text { Analysis of Double Shell Slurry. Internal } \\
\text { Letter \# } 65611-84-053 \text { to DJ Fisher dated } \\
\text { 4/5/84, Rockwell Hanford Operations, } \\
\text { Richland, WA. }\end{array}$ & $\mathrm{NA}$ & Blended Slurry & Reject & $\begin{array}{l}\text { Outdated document, (prior to Q2 1985). } \\
\text { Results of organic analysis on DSS } \\
\text { (blended) samples as part of slurry growth } \\
\text { studies. Initial DSS boildown composition } \\
\text { from reference \#AN-104-1. }\end{array}$ \\
\hline AN-105-2 & $5 / 10 / 84$ & $\begin{array}{l}\text { Jansky MT. 1984. Laboratory Support } \\
\text { for Upcoming 242-A Evaporator Campaign, } \\
\text { Run 84-5. Internal memo \# 65453-84-134 } \\
\text { to EG Gratny dated 5/10/84, Rockwell } \\
\text { Hanford Operations, Richland, WA. }\end{array}$ & NA & Supernatant & Reject & $\begin{array}{l}\text { Outdated document, (prior to } Q 2 \text { 1985). } \\
\text { Lab support for upcoming } 242-\mathrm{A} \text { evaporator } \\
\text { campaign } 84-5 \text {. Analytical results of two } \\
\text { samples (R9839 and R9840) }\end{array}$ \\
\hline AN-105-3 & $11 / 9 / 84$ & $\begin{array}{l}\text { Mauss BM. 1984. Chemical } \\
\text { Compositions of 102-AY, 101-AW, 105-AN, } \\
\text { and 104-AW. Infernal memo \# 65453-84- } \\
348 \text { to EG Gratny dated 11/9/84, Rockwell } \\
\text { Hanford Operations, Richland, WA }\end{array}$ & NA & Supernatant & Reject & $\begin{array}{l}\text { Outdated document, (prior to Q2 1985). } \\
\text { Chemical composition of AN105. } \\
\text { Analytical results for AN105 sample (no ID) } \\
\text { and no details about sample. }\end{array}$ \\
\hline
\end{tabular}




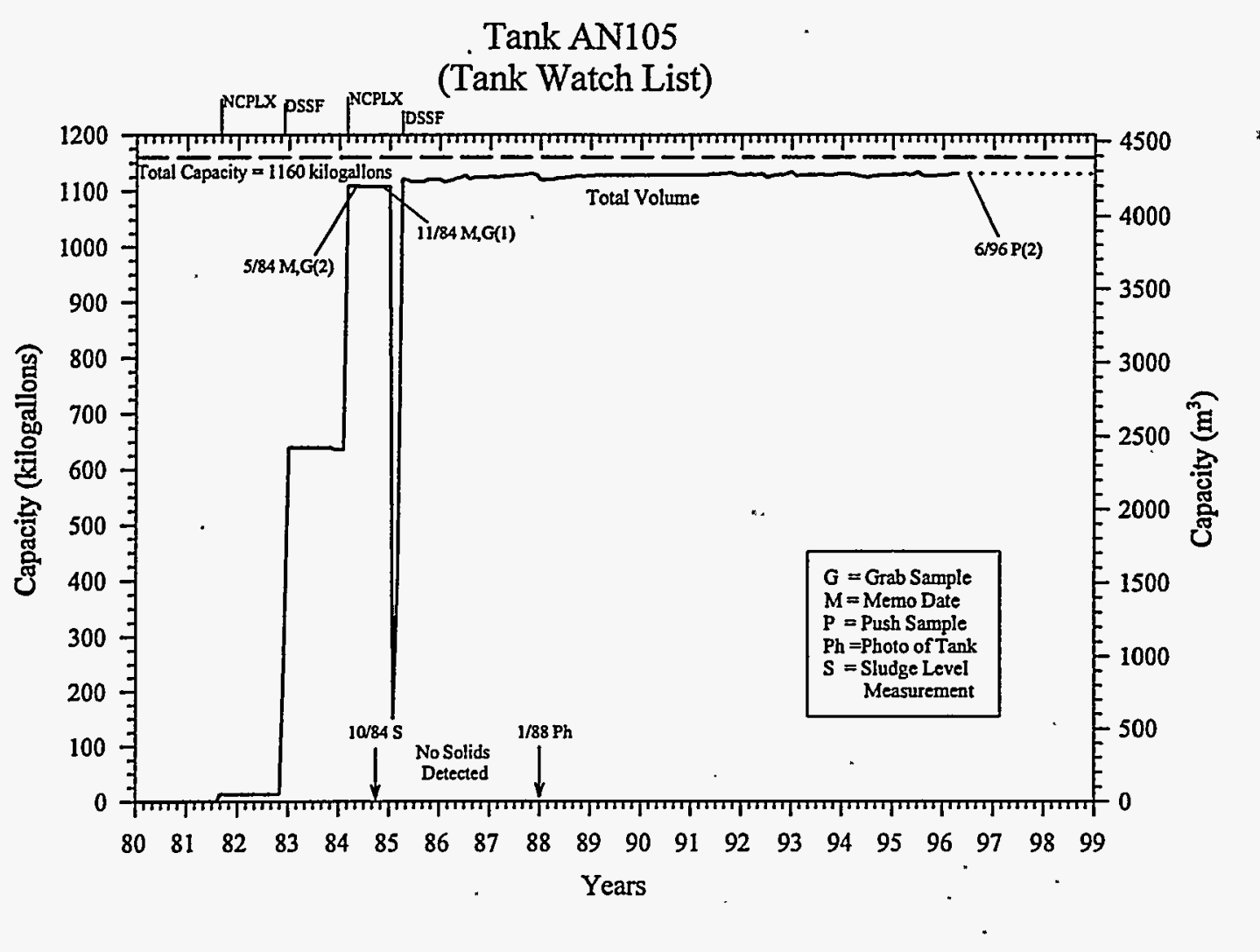

Figure 6.1. Tank Waste Volume and Sampling Event Graph for Tank AN105

The last transfer to this tank, in January 1985 , was about $3785 \mathrm{~m}^{3}$ (1000 kgal) of waste from AW102 (see Appendix B). Tank level history data for AN105 indicates that the waste type did not change with the last transfer in January 1985 (waste type remained non-complexed (NCPLX)). However, in - October 1986, the waste type was changed to DSSF for unknown reasons (the tank volume had remained unchanged between during the preceding month). The waste was probably misclassified after the last transfer and was corrected in October. Supporting laboratory studies were performed an evaporator run for tank AN105 (AN-105-2). Therefore, the large transfer in early 1985 significantly changed the tank waste composition. If this evaporator campaign occurred (supporting documentation unavailable for review), it was not a simple transfer of waste from AN105 to AW102 and back to AN105 (as suggested by Goheen-see appendix B). - Slight changes in level recorded April 1985 have been attributed to wash water additions or slurry growth from gas generation (Brevick, Gaddis, and Pickett 1995). 


\subsection{AN107}

\subsection{Tank Summary}

Tank AN107 has a waste volume of $4005 \mathrm{~m}^{3}$ (1058 kgal), which includes $507 \mathrm{~m}^{3}$ (134 kgal) of solids. The waste in this tank is designated as complexed concentrate (CC) waste. The tank began service in September 1981 and continued to receive non-complexed waste from tank AN102 until June 1983; the only other documented additions were waste from AZ102 in October and November 1983.

\subsection{Reference Summary}

Table 7.1 summarizes the references from which the characterization data for waste in tank AN107 were retrieved. The summary includes the reference number from Kaiser's Master Set data, memo date, the reference citation, sample date and type, whether the data in the reference were accepted for use in the Subset data, and comments about the reference.

\subsection{Waste Volume and Sampling Events}

The waste volume and tank sampling event plot is shown in Figure 7.1. Tank AN107 contains CC waste from tanks AN102 and AZ102. It is scheduled to have caustic added to it in 1997, after which several samplings to measure hydroxide concentrations are planned. The total volume appears to be decreasing slightly due to evaporation. The step function in the solids volume plot is an artifact of the measuring intervals: The solids volume was last measured in June 1989.

\subsection{Master Set}

All of the analytical data acquired by Kaiser were included in the Master Set. The Master Set data were compared to original laboratory reports to verify that the numbers and units were transcribed correctly, and that the units were converted correctly to the standard $\mu \mathrm{g} / \mathrm{L}$ format. Dates and reference numbers were also checked for accuracy.

\subsection{Subset}

Valid data were extracted from 8 of a total of 22 references. The TRAC, Braun, or Wastren data in the Master Set were not used. The HTCE data were kept but not factored into the $\mathrm{min} / \mathrm{max} / \mathrm{best}$ estimate. "Less than" values were kept and were used for the $\mathrm{min} / \mathrm{max} / \mathrm{best}$ estimate only if they were the only values available for a species. Values for duplicate samples were averaged, otherwise different sample aliquots were kept as individual analyses. 
Table 7.1. Summary of Source Documents Containing Waste Characterization Data for AN107

\begin{tabular}{|c|c|c|c|c|c|c|}
\hline $\begin{array}{c}\text { Kaiser } \\
\text { Reference } \\
\text { Number }\end{array}$ & $\begin{array}{l}\text { Memo } \\
\text { Date }\end{array}$ & Source Document* & $\begin{array}{l}\text { Sample } \\
\text { Date }\end{array}$ & $\begin{array}{l}\text { Sample } \\
\text { Type }\end{array}$ & $\begin{array}{l}\text { Accept/ } \\
\text { Reject }\end{array}$ & Comments \\
\hline AN 107-1 & $10 / 10 / 80$ & $\begin{array}{l}\text { Herting DL. 1980. Analysis of Samples from Tank 107- } \\
\text { AN and 104-AW. Internal letter \# 65453-80-298 to DR } \\
\text { Groth dated 10/10/80, Rockwell Hanford Operations, } \\
\text { Richland, WA. }\end{array}$ & Unavailable & Supernate & Accept & $\begin{array}{l}\text { Contains data on samples } 5439 \& \\
5440 \text { for } \mathrm{OH}, \mathrm{NO}_{2}^{-}, \mathrm{pH} \text {, total } \\
\text { alpha \& total beta. }\end{array}$ \\
\hline AN107-2 & $4 / 23 / 84$ & $\begin{array}{l}\text { Jansky MT. 1984. Tank } 107 A N \text { Samples. Internal Letter } \\
\text { \# } 65453-84-113 \text { to RB Bendixsen dated } 4 / 23 / 84 \text {, Rockwell } \\
\text { Hanford Operations, Richland, WA. }\end{array}$ & $3 / 30 / 84$ & Supernate & Accept & $\begin{array}{l}\text { Data on samples, } \mathrm{R} 8843 \text { \& } \\
\mathrm{R} 8844, \text { for HEDTA, EDTA, } \\
\text { TOC, } \mathrm{NO}_{2} ; \text { \& } \mathrm{NO}_{3}: \text { Results are } \\
\text { listed as slurry, apparently not } \\
\text { filtered. Amount of solids } \\
\text { undetermined. }\end{array}$ \\
\hline \multirow[t]{2}{*}{ AN107-3 } & $2 / 28 / 85$ & $\begin{array}{l}\text { Bratzel DR. 1985. Characterization of Complexant } \\
\text { Concentrate Supenatant. Internal letter \# 65453-85-041 to } \\
\text { JN Appel dated 2/28/95, Rockwell Hanford Operations, } \\
\text { Richland, WA. }\end{array}$ & Unavailable & Supernate & Accept & $\begin{array}{l}\text { Table } 2 \text { contains data for cations, } \\
\text { anions, TOC, specific gravity, } \\
\text { Table } 3 \text { contains radionuclide } \\
\text { data. Both for supernate and } \\
\text { centrate for samples R- } 3637 \text { \& } \\
\text { R3638. }\end{array}$ \\
\hline & $3 / 14 / 85$ & $\begin{array}{l}\text { Bratzel DR. 1985. Characterization of Complexant } \\
\text { Concentrate Solids from Tanks 107-AN, 102-AN, and 101- } \\
A Y \text {. Internal letter \# 65453-85-053 to JN Appel dated } \\
\text { 3/14/95, Rockwell Hanford Operations, Richland, WA. }\end{array}$ & Unavailable & Solids & Reject & $\begin{array}{l}\text { Data on solids from samples } \\
\text { R3637 \& R3638. }\end{array}$ \\
\hline AN107-4 & $12 / 4 / 86$ & $\begin{array}{l}\text { Herting DL. 1986. Hydroxide Concentration in Tank } \\
\text { 107-AN. Internal letter \# } 65453-86-155 \text { to DA Reynolds } \\
\text { dated 12/4/86, Rockwell Hanford Operations, Richland, } \\
\text { WA. }\end{array}$ & Unavailable & Supernate & Accept & $\begin{array}{l}\text { Data is given in Table } 2 \text { for } \mathrm{pH}, \\
\mathrm{OH}, \mathrm{TIC}, \mathrm{NO}_{2}^{-}, \mathrm{NO}_{3}^{-}, \mathrm{SO}_{4}^{-2} \\
\mathrm{PO}_{4}^{-3}, \mathrm{Na}, \mathrm{Al}, \mathrm{Fe}, \mathrm{TOC} \text { and total } \\
\text { alpha. }\end{array}$ \\
\hline
\end{tabular}




\begin{tabular}{|c|c|c|c|c|c|c|}
\hline $\begin{array}{l}\text { Kaiser } \\
\text { Reference } \\
\text { Number }\end{array}$ & $\begin{array}{l}\text { Memo } \\
\text { Date }\end{array}$ & Source Document* & $\begin{array}{l}\text { Sample } \\
\text { Date }\end{array}$ & $\begin{array}{l}\text { Sample } \\
\text { Type }\end{array}$ & $\begin{array}{l}\text { Accept/ } \\
\text { Reject }\end{array}$ & Comments \\
\hline AN107-5 & $7 / 6 / 88$ & $\begin{array}{l}\text { Prignano AL. 1988. Tanks 102-AN and 107-AN Viscosity } \\
\text { and Percent Settled Solids Determination. WHC-IP-0711. } \\
\text { Internal memo \# 12221-PCL88-155 to DE Scully and EC } \\
\text { Vogt dated 7/6/88, Westinghouse Hanford Company, } \\
\text { Richland, WA. }\end{array}$ & Unavailable & Slurry & Reject & $\begin{array}{l}\text { Does not contain any chemical or } \\
\text { radionuclide data. Does contain } \\
\text { data on viscosity and \% settled } \\
\text { solids from R8569 \& R8374. }\end{array}$ \\
\hline AN107-6 & $5 / 16 / 90$ & $\begin{array}{l}\text { Hall B. 1990. Toxic Metals Concentrations of DSS and } \\
\text { CC Containing Tanks. DSI to DD Stepnewski dated } \\
5 / 16 / 90 \text {, Westinghouse Hanford Company, Richland, WA. }\end{array}$ & Unavailable & Supernate & Accept & $\begin{array}{l}\text { Contains values for } \mathrm{Ba}, \mathrm{Cd}, \mathrm{Cr} \text {, } \\
\text { and } \mathrm{Pb} \text {. }\end{array}$ \\
\hline AN107-7 & $6 / 29 / 90$ & $\begin{array}{l}\text { Washington LO. } 1990 \text {. Chemical Addition to Tank 107- } \\
\text { AN. Internal memo \# 16220-PCL90-079 to JP Harris } \\
\text { dated } 6 / 29 / 90 \text {, Westinghouse Hanford Company, Richland, } \\
\text { WA. }\end{array}$ & Unavailable & Supernate & Accept & $\begin{array}{l}\text { Table } 1 \text { gives data for cations, } \\
\text { anions, TOC, TIC and Cs137. } \\
\text { Only used data for Exp. A } \\
\text { Control and Exp. C Control. }\end{array}$ \\
\hline \multirow[t]{4}{*}{ AN107-8 } & $8 / 10 / 94$ & $\begin{array}{l}\text { Herting DL. 1994. Characterization of Sludge Samples } \\
\text { From Tank } 24 I-A N-107 \text {. Internal memo \# 8E110-PCL94- } \\
064 \text { to KG Carothers dated } 8 / 10 / 94 \text {, Westinghouse } \\
\text { Hanford Company, Richland, WA. }\end{array}$ & $5 / 13 / 94$ & Centrate & Accept & $\begin{array}{l}\text { Table 4, contains data for cations, } \\
\text { anions, TOC, TIC, Sr-90 \& Cs- } \\
\text { 137. }\end{array}$ \\
\hline & $6 / 6 / 89$ & $\begin{array}{l}\text { Reynolds DA. 1989. Reaction Kinetics in Complexed } \\
\text { Concentrate. Internal Memo \# 13314-89-050 to DL } \\
\text { Herting dated 6/6/89, Westinghouse Hanford Company, } \\
\text { Richland, WA. }\end{array}$ & Unavailable & & Reject & $\begin{array}{l}\text { Contains data from other tanks } \\
\text { and data previously reported for } \\
\text { AN107. }\end{array}$ \\
\hline & $5 / 13 / 93$ & $\begin{array}{l}\text { Herting DL. 1993. Tank 241-AN-107 Caustic Demand. } \\
\text { Internal memo \# 12110-PCL93-042 to KG Carothers dated } \\
\text { 5/13/93, Westinghouse Hanford Company, Richland, WA. }\end{array}$ & Unavailable & Supernate & $\begin{array}{l}\text { Accept } \\
\text { (for } \\
\text { TIC } \\
\text { data) }\end{array}$ & $\begin{array}{l}\text { Table A1, data on cations, anions, } \\
\text { Cs, Pu, Am, Np \& Sr from } \\
\text { R2686. R3154 \& R3155. } \\
\text { Contains same data as AN-107-8 }\end{array}$ \\
\hline & $12 / 21 / 94$ & $\begin{array}{l}\text { Herting DL. 1994. Acetate, Formate, and Oxalate in } \\
\text { Tank 107-AN. Internal memo \# 8E110-PCL94-067 to KG } \\
\text { Carothers dated 12/21/94, Westinghouse Hanford } \\
\text { Company, Richland, WA. }\end{array}$ & Unavailable & Centrate & Reject & $\begin{array}{l}\text { Contains data on acetate, formate } \\
\text { and oxalate and TOC (Table 2). } \\
\text { Not used because these ions were } \\
\text { not called out. }\end{array}$ \\
\hline
\end{tabular}




\begin{tabular}{|c|c|c|c|c|c|c|}
\hline $\begin{array}{c}\text { Kaiser } \\
\text { Reference } \\
\text { Number }\end{array}$ & $\begin{array}{l}\text { Memo } \\
\text { Date }\end{array}$ & Source Document* & $\begin{array}{l}\text { Sample } \\
\text { Date }\end{array}$ & $\begin{array}{l}\text { Sample } \\
\text { Type }\end{array}$ & $\begin{array}{l}\text { Accept/ } \\
\text { Reject }\end{array}$ & Comments \\
\hline & $5 / 8 / 84$ & $\begin{array}{l}\text { Bendixsen RB. } 1984 \text { Complexant Concentration in } \\
107-A N \text { Tank. Internal letter\# } 65950-84-300 \text { to LH Rogers } \\
\text { dated } 5 / 8 / 84 \text {, Rockwell Hanford Operations, Richland, } \\
\text { WA. }\end{array}$ & $3 / 30 / 84$ & Slurry? & Reject & $\begin{array}{l}\text { Data is a comparison with AZ101 } \\
\text { both before and after addition of } \\
234 \text { inches of AZ101 waste to } \\
\text { AN107. }\end{array}$ \\
\hline & $3 / 24 / 87$ & $\begin{array}{l}\text { Reynolds DA. 1987. 107/102-AN Chemical Additions. } \\
\text { Internal letter \# 65611-87-058 to DE Bratzel dated } \\
\text { 3/24/87, Rockwell Hanford Operations, Richland, WA. }\end{array}$ & Unavailable & & Reject & $\begin{array}{l}\text { Calculation of chemicals to add } \\
\text { for corrosion compliance. Only } \\
\text { has operating specifications. }\end{array}$ \\
\hline & $3 / 30 / 87$ & $\begin{array}{l}\text { Herting DL. 1987. Chemical Additions to Tanks } \\
\text { 107/102. Internal letter \# } 65453-87-044 \text { to DA Reynolds } \\
\text { dated 3/30/87, Rockwell Hanford Operations, Richland, } \\
\text { WA. }\end{array}$ & Unavailable & Supernate & Reject & $\begin{array}{l}\text { Reply to memo dated } 3 / 24 / 87 \\
\text { with calculations and previously } \\
\text { reported data. Data from AN107- } \\
4\end{array}$ \\
\hline & $2 / 25 / 88$ & $\begin{array}{l}\text { Reynolds DA. 1988. Recommended Corrosion Control in } \\
241-A N-107,102 \text { Tanks. Internal memo \# } 13314-88-034 \\
\text { to GL Dunford dated } 2 / 25 / 88 \text {, Westinghouse Hanford } \\
\text { Company, Richland, WA. }\end{array}$ & Unavailable & & Reject & $\begin{array}{l}\text { Corrosion control for AN107, no } \\
\text { chemical or radionuclide data. }\end{array}$ \\
\hline & $10 / 15 / 80$ & $\begin{array}{l}\text { Jansky MT. 1980. Tank 107-AN Liquid Rinse Samples } \\
\text { Analyses. Internal letter \# } 65453-80-303 \text { to DR Groth } \\
\text { dated 10/15/80, Rockwell Hanford Operations, Richland, } \\
\text { WA. }\end{array}$ & Unavailable & $\begin{array}{l}\text { Liquid } \\
\text { rinse }\end{array}$ & Reject & $\begin{array}{l}\text { Rinse of tank prior to transfer of } \\
\text { waste into it. Not representative } \\
\text { of waste currently in this tank. }\end{array}$ \\
\hline & $12 / 12 / 80$ & $\begin{array}{l}\text { Jansky MT and DL Herting. 1980. Scrapings from } \\
107 A N \& \text { 104AW. Internal letter \# } 65453-80-375 \text { to J. } \\
\text { Scofield dated } 12 / 12 / 80 \text {, Rockwell Hanford Operations, } \\
\text { Richland, WA. }\end{array}$ & $\begin{array}{c}\text { Unavailable } \\
.\end{array}$ & Solids & Reject & Solids, no liquid samples. \\
\hline & $5 / 17 / 85$ & $\begin{array}{l}\text { Bratzel DR. 1985. Quarterly Status Report on } \\
\text { Complexant Concentrate Laboratory Studies. Internal } \\
\text { letter \# 65453-85-100 to JN Appel dated 5/17/85, } \\
\text { Rockwell Hanford Operations, Richland, WA. }\end{array}$ & Unavailable & Supernate & Reject & $\begin{array}{l}\text { Quarterly status report, data } \\
\text { contained in other reports. }\end{array}$ \\
\hline
\end{tabular}




\begin{tabular}{|c|c|c|c|c|c|c|}
\hline $\begin{array}{l}\text { Kaiser } \\
\text { Reference } \\
\text { Number }\end{array}$ & $\begin{array}{l}\text { Memo } \\
\text { Date }\end{array}$ & Source Document* & $\begin{array}{c}\text { Sample } \\
\text { Date }\end{array}$ & $\begin{array}{c}\text { Sample } \\
\text { Type }\end{array}$ & $\begin{array}{c}\text { Accept/ } \\
\text { Reject }\end{array}$ & Comments \\
\hline . & $11 / 11 / 92$ & $\begin{array}{l}\text { Wilkin NE. 1992. Potential Addition of Tanks to Watch } \\
\text { List. Internal memo \# } 7 \mathrm{~K} 210-92-457, \text { Rev. } 1 \text { to JC } \\
\text { Fulton dated } 11 / 11 / 92 \text {, Westinghouse Hanford Company, } \\
\text { Richland, WA. }\end{array}$ & Unavailable & & Reject & $\begin{array}{l}\text { Comparison of tanks for addition } \\
\text { to watch list, no new data. }\end{array}$ \\
\hline & & $\begin{array}{l}\text { Toste AP. Hydrophilic Organics Identified in Organic } \\
\text { Complexant Waste from Tank } 107 \text { AN. Memo to LM } \\
\text { Sasaki, Pacific Northwest Laboratory, Richland, WA. }\end{array}$ & Unavailable & & Reject & $\begin{array}{l}\text { Data on citric acid, EDTA, } \\
\text { HEDTA, NTA, and chelator } \\
\text { fragments. }\end{array}$ \\
\hline & $5 / 15 / 90$ & $\begin{array}{l}\text { Bechtold DB. 1990. DSC of Tank 107-AN Supernate. } \\
\text { Internal memo \# 12712-PCL90-067 to SE Kelly dated } \\
\text { 5/15/90, Westinghouse Hanford Company, Richland, WA. }\end{array}$ & $5 / 4 / 90$ & Supernate & Reject & $\begin{array}{l}\text { Differential scanning calorimetry } \\
\text { data. No chemical or } \\
\text { radionuclide data. }\end{array}$ \\
\hline
\end{tabular}

* Many source documents are not cleared for public release. They are cited to provide traceablility to the original data. 


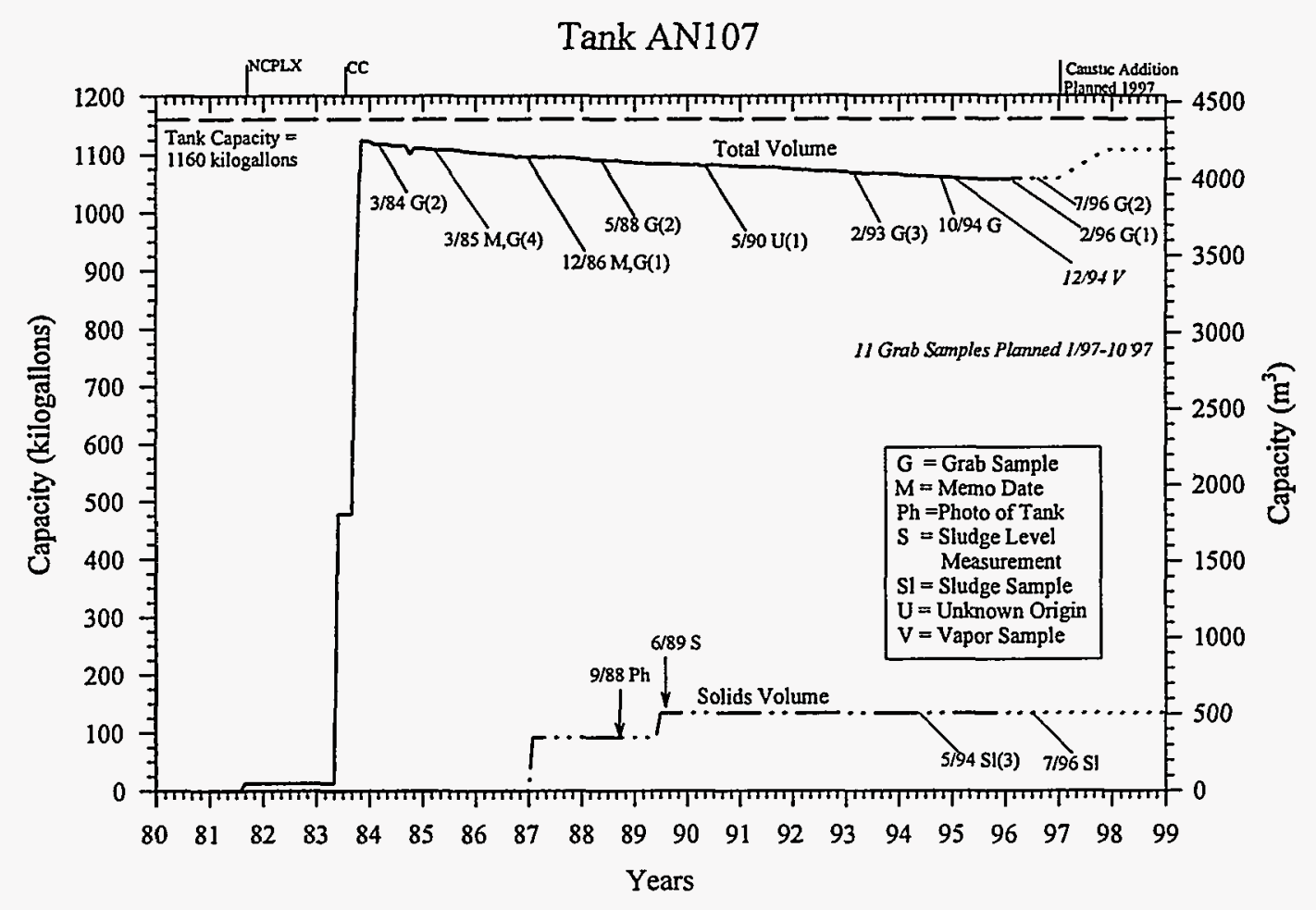

Figure 7.1. Tank Waste Volume and Sampling Event Graph for Tank AN107

The only data outside of the valid Subset that required validation were the values for TIC (Total Inorganic Carbon) or carbonate $\left(\mathrm{CO}_{3}^{-2}\right)$. The values used were from the memo dated 5/13/93. (a) $^{2}$

\section{6 $\mathrm{Min} / \mathrm{Max} / \mathrm{BE}$}

The lowest and highest concentrations in the Subset were designated as the minimum and maximum, respectively. The best estimate was determined by calculating an average of all the valid data points in the Subset.

\subsection{Organics}

Organic speciation (acetate, formate and oxalate) in the sludge and centrate for Tank AN107 have been reported by Herting ${ }^{(b)}$. Acetate was $33 \%$, formate was $6.7 \%$ and oxalate was

(a) Herting DL. 1993. Tank 241-AN-107 Caustic Demand. Internal memo \# 12110-PCL93-042 to KG Carothers dated 5/13/93, Westinghouse Hanford Company, Richland, WA.

(b) Herting DL. 1994. Acetate, Formate, and Oxalate in Tank 107-AN. Internal memo to KG Carothers dated 12/21/94, Westinghouse Hanford Company, Richland, WA. 
$8.8 \%$ of TOC for the supernate.

\subsection{Physical Properties}

Physical property measurements were made on the sludge in the tank. Particle size measurements were performed in June 1990 (AN-107-7). Some sludge characterization observations were presented in a memo from Herting (AN-107-8).

The supernate samples varied in their visual characterization. Two samples taken in 1980 indicated that the liquid above the solids was colorless while the solids were rust colored (AN-107-1). Samples obtained on March 30, 1984 were extremely dark and therefore difficult to assess for the amount of solid material in the sample (AN-107-2). In 1988, two samples were described as brown-amber in color (AN-107-5).

\subsection{Limitations/Concerns}

This tank has been characterized much more thoroughly than Tank AN102. 


\subsection{AP102}

\subsection{Tank Summary}

Tank AP102 has a total volume of $4152 \mathrm{~m}^{3}$ (1097 kgal) and contains concentrated phosphate waste (CP) (Hanlon 1996). Concentrated phosphate waste originated from the decontamination of the $\mathrm{N}$ reactor in the $100 \mathrm{~N}$ Area. The waste volume is entirely supernatant. The tank is designated as a Grout Feed Tank (GRTFD), even though the grout program is no longer in effect.

\subsection{Reference Summary}

Table 8.1 summarizes the references from which the characterization data for waste in tank AP102 were retrieved. The summary includes the reference number from Kaiser's Master Set data, memo date, the reference citation, sample date and type, whether the data in the reference were accepted for use in the Subset data, and comments about the reference. Only one document AP-102-3 was found to have valid data; however those data were sufficient to characterize the waste in AP102.

\subsection{Waste Volume and Sampling Events}

The waste volume and tank sampling event plot is shown in Figure 8.1. Tank AP102 contains CP waste that was transferred from tank AN106. The waste volume is near the capacity of the tank. No further transactions are planned before the waste is sent to pretreatment. No measurable solids were found in the tank at the last solids measurement (July 1989). The figure shows only one sampling event since the last major transfer. Of 25 samples taken over a three day period, 18 were analyzed for inorganic components and 7 for organic components (Welsh 1994a).

\subsection{Master Set}

The original Kaiser Master Set data base for tank AP102 waste was entirely reconstructed from the only valid original source document (AP-102-3). Differences between the original and reconstructed Master Sets include the following:

- Approximately 150 points were eliminated because the data were outdated. Other data could not be traced to the original source or were wrongly typed or entered twice.

- Hundreds of new data points given in the original source but left out of the database were entered.

- Cells were corrected to include densities and to change reflect 'less-than' values--for example, <2.2 E3 would replace the incorrect value of $2.2 \mathrm{E} 3$. 
Table 8.1. Summary of Source Documents Containing Waste Characterization Data for AP102

\begin{tabular}{|l|l|l|l|l|l|l||}
\hline $\begin{array}{l}\text { Kaiser } \\
\text { Referenc } \\
\text { e } \\
\text { Number }\end{array}$ & $\begin{array}{c}\text { Memo } \\
\text { Date }\end{array}$ & Source Document* & $\begin{array}{c}\text { Sample } \\
\text { Date }\end{array}$ & $\begin{array}{c}\text { Sample } \\
\text { Type }\end{array}$ & $\begin{array}{c}\text { Accept/ } \\
\text { Reject }\end{array}$ & Comments \\
\hline \hline AP-102-1 & $6 / 8 / 88$ & $\begin{array}{l}\text { Winters WI. 1988. Final Report on Tank 241-AP-102 } \\
\text { Characterization. Internal Memo \#12221-ASL88-154 to } \\
\text { WG Richmond dated 6/8/88, Westinghouse Hanford. } \\
\text { Company, Richland, WA. }\end{array}$ & Supernatant & Reject & Outdated information. \\
\hline AP-102-2 & $2 / 11 / 93$ & $\begin{array}{l}\text { Hendrickson DW. 1993. Program Campaign 102 Feed } \\
\text { Characterization and Test Plan. WHC-SD-WM-TP-136, } \\
\text { Westinghouse Hanford Company, Richland, WA. }\end{array}$ & & Reject & Projected values only. \\
\hline AP-102-3 & $5 / 19 / 94$ & $\begin{array}{l}\text { Welsh TL. 1994a. Tank 241-AP-102 Characterization } \\
\text { and Grout Product Test Results. WHC-SD-WM-TRP- } \\
168, \text { Westinghouse Hanford Company, Richland, WA. }\end{array}$ & $4 / 28-30 / 93$ & Supernatant & Accept & $\begin{array}{l}\text { Information given in this report was } \\
\text { found to be enough to characterize } \\
\text { the waste in AP102. }\end{array}$ \\
\hline
\end{tabular}




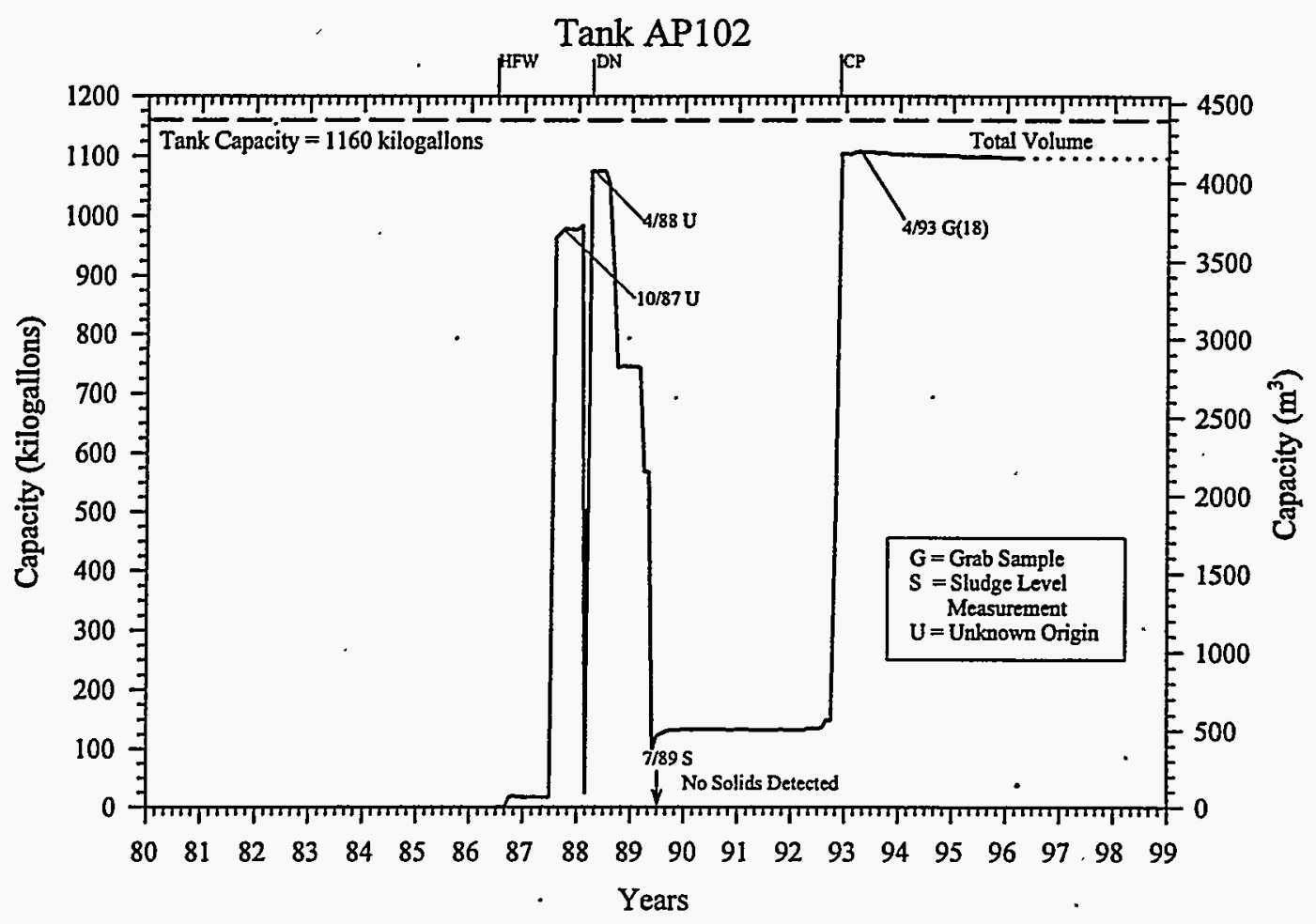

Figure 8.1. Tank Waste Volume and Sampling Event Graph for Tank AP102

- The heading of the S/L column was change to $\mathrm{L}$. This tank does not contain solids. Solids were entered in the database due to a misinterpretation of the analysis results. Sodium phosphate precipitated from the samples collected for AP102; its solubility is low at ambient temperatures in the analytical laboratory. New samples were then collected and kept at $40^{\circ} \mathrm{C}$. Because many analyses used acid digestion and dilution, the precipitate had little influence on the results. Information regarding the homogeneous liquid contents of AP102 can be found in the report (Welsh 1994a).

- New columns were inserted for sample date, analysis date, tank sample number, laboratory sample number, half-life and molecular weights, and comments. The laboratory sample number is the only way to trace the data to the original source. The TCD numbers were not useful for such tracing.

- Special attention was paid to the "less than values" given in the data base. The < character causes problems when calculations need to be done. Instead the cell was formatted as "less than the number entered". When copying information from this cell or using this cell in a calculation, attention should be paid to make sure the less than format is carried to the receiving cell, where the number is either being pasted or used in a calculation. An Excel macro was written by Ralph Leonard (ANL) to eliminate this problem.

It was recommended that the changes in format and the inclusion of additional column be 
applied to the other tanks as well.

\subsection{Subset}

For this tank the reconstructed Master Set and the Subset were the same because the Master Set was reconstructed with valid data only. See the description in the previous section.

\section{6 $\mathrm{Min} / \mathrm{Max} / \mathrm{BE}$}

The lowest concentration in the Subset was selected as the minimum value; the highest concentration in the Subset was selected as the maximum value. The best estimate was the average of all the Subset values. Less than values were not averaged with other values to determine the best estimate.

\subsection{Organics}

Extensive analyses of organic constituents, including complexant, volatile, and semivolatile compound concentrations were conducted on 7 samples obtained from AP102 in April 1994 (Welsh 1994a). The complexants analyzed include EDTA ( 87 to $780 \mathrm{mg} / \mathrm{L}$ ), HEDTA (23 to $210 \mathrm{mg} / \mathrm{L}$ ), citrate $(400$ to $670 \mathrm{mg} / \mathrm{L})$, glycolate $(1100 \mathrm{mg} / \mathrm{L})$, and oxalate $(700$ to $800 \mathrm{mg} / \mathrm{L})$. The volatile compounds, acetone $(230 \mu \mathrm{g} / \mathrm{L})$ and 2-butanone $(590 \mu \mathrm{g} / \mathrm{L})$ were identified in only one sample, and toluene $(43-140 \mu \mathrm{g} / \mathrm{L})$ was detected in two. Both acetone and toluene were below the contract required quantitation limit (CRQL). Analysis of semivolatile compounds involved acidifying the waste samples and consequent formation of a precipitate. A separate analysis of the precipitate detected no semivolatile compounds. Semivolatile compounds, 1-butanol (370-820 $\mu \mathrm{g} / \mathrm{L})$ and bis(2ethylhexyl)phthalate, were detected in six samples at levels below the CRQL.

\subsection{Physical Properties}

Although, the waste in AP102 does not contain any solids, sodium phosphate precipitated at ambient temperatures from samples not maintained at the tank temperature $\left(40^{\circ} \mathrm{C}\right)$. Many analytical measurements used acid digestion, and the precipitate had little influence on the results. The tank solution is a CP waste with a density of $1.2 \mathrm{~g} / \mathrm{mL}$ and a $\mathrm{pH}$ of 13.7 (calculated from tank hydroxide concentration). The water concentration, as determined by oven-drying the samples at $120^{\circ} \mathrm{C}$ for $16-20$ hours, is $75 \%$.

\subsection{Limitations/Concerns}

The waste in tank AP102 is one of the best characterized wastes included in the Privatization effort. The information in the database developed at ANL is published in the report Tank 241-AP-102 Characterization and Grout Product Test Results (Welsh 1994a). Information from this report was sufficient to characterize AP102 waste. The sampling dates (April 28-30, 1993), and analysis dates (May through December 1993) are current; no major transfers have occur since fourth quarter 1992. 
Table 8.2. Semivolatile and Volatile Organic Compounds

\begin{tabular}{|c|c|c|}
\hline Analyte & Sample Number & $\begin{array}{l}\text { Reported Value } \\
\quad \text { (ug/L) }\end{array}$ \\
\hline \multicolumn{3}{|c|}{ Semivolatile Organic Compounds } \\
\hline 1-Butanol & $\begin{array}{l}\text { G300-1 } \\
\text { G300-2 } \\
\text { G304 } \\
\text { G312 } \\
\text { G314 } \\
\text { G316-1 } \\
\text { G316-2 } \\
\text { G320 } \\
\text { G321 } \\
\text { G324 }\end{array}$ & $\begin{array}{c}370^{*} \\
470^{*} \\
\text { ND } \\
750^{*} \\
820^{*} \\
600^{*} \\
690^{*} \\
730^{*} \\
510^{*} \\
\text { ND }\end{array}$ \\
\hline Pyridine & $\begin{array}{l}\text { G300-1, G300-2, G304, G312, G314, G316-1, G316-2, } \\
\text { G320, G321, G324 }\end{array}$ & ND \\
\hline 1,4-Dichlorobenzene & $\begin{array}{l}\text { G300-1, G300-2, G304, G312, G314, G316-1, G316-2, } \\
\text { G320, G321, G324 }\end{array}$ & ND \\
\hline 1,2-Dichlorobenzene & $\begin{array}{l}\text { G300-1, G300-2, G304, G312, G314, G316-1, G316-2, } \\
\text { G320, G321, G324 }\end{array}$ & ND \\
\hline 2-Methylphenol & $\begin{array}{l}\text { G300-1, G300-2, G304, G312, G314, G316-1, G316-2, } \\
\text { G320, G321, G324 }\end{array}$ & ND \\
\hline 4-Methylphenol & $\begin{array}{l}\text { G300-1, G300-2, G304, G312, G314, G316-1, G316-2, } \\
\text { G320, G321, G324 }\end{array}$ & ND \\
\hline Hexachloroethane & $\begin{array}{l}\text { G300-1, G300-2, G304, G312, G314, G316-1, G316-2, } \\
\text { G320, G321, G324 }\end{array}$ & ND \\
\hline Nitrobenzene & $\begin{array}{l}\text { G300-1, G300-2, G304, G312, G314, G316-1, G316-2, } \\
\text { G320, G321, G324 }\end{array}$ & ND \\
\hline Hexachlorobutadiene & $\begin{array}{l}\text { G300-1, G300-2, G304, G312, G314, G316-1, G316-2, } \\
\text { G320, G321, G324 }\end{array}$ & ND \\
\hline 2,4,6-Trichlorophenol & $\begin{array}{l}\text { G300-1, G300-2, G304, G312, G314, G316-1, G316-2, } \\
\text { G320, G321, G324. }\end{array}$ & ND \\
\hline 2,4,5-Trichlorophenol & $\begin{array}{l}\text { G300-1, G300-2, G304, G312, G314, G316-1, G316-2, } \\
\text { G320, G321, G324 }\end{array}$ & ND \\
\hline 2,4-Dinitrotoluene & $\begin{array}{l}\text { G300-1, G300-2, G304, G312, G314, G316-1, G316-2, } \\
\text { G320, G321, G324 }\end{array}$ & ND \\
\hline Diethylphthalate & $\begin{array}{l}\text { G300-1, G300-2, G304, G312, G314, G316-1, G316-2, } \\
\text { G320, G321, G324 }\end{array}$ & ND \\
\hline Tributylphosphate & $\begin{array}{l}\text { G300-1, G300-2, G304, G312, G314, G316-1, G316-2, } \\
\text { G320, G321, G324 }\end{array}$ & ND \\
\hline
\end{tabular}




\begin{tabular}{|c|c|c|}
\hline Analyte & Sample Number & $\begin{array}{l}\text { Reported Value } \\
\quad(u g / L)\end{array}$ \\
\hline Hexachlorobenzene & $\begin{array}{l}\text { G300-1, G300-2, G304, G312, G314, G316-1, G316-2, } \\
\text { G320, G321, G324 }\end{array}$ & ND \\
\hline Pentachlorophenol & $\begin{array}{l}\text { G300-1, G300-2, G304, G312, G314, G316-1, G316-2, } \\
\text { G320, G321, G324 }\end{array}$ & ND \\
\hline Di-n-octylphthalate & $\begin{array}{l}\text { G300-1, G300-2; G304, G312, G314, G316-1, G316-2, } \\
\text { G320, G321, G324 }\end{array}$ & ND \\
\hline \multicolumn{3}{|c|}{ Volatile Organic Compounds } \\
\hline Vinyl Chloride & $\begin{array}{l}\text { G300, G304, G312, G314, G314D, G316, G320, G321, } \\
\text { G324 }\end{array}$ & ND \\
\hline Methylene Chloride & $\begin{array}{l}\text { G300, G304, G312, G314, G314D, G316, G320, G321, } \\
\text { G324 }\end{array}$ & ND \\
\hline Acetone & $\begin{array}{l}\text { G314 } \\
\text { G300, G304, G312, G314D, G316, G320, G321, G324 }\end{array}$ & $\begin{array}{l}230 * \\
\text { ND }\end{array}$ \\
\hline Carbon Disulfide & $\begin{array}{l}\text { G300, G304, G312, G314, G314D, G316, G320, G321, } \\
\text { G324 }\end{array}$ & ND \\
\hline 1,1-Dichloroethene & $\begin{array}{l}\text { G300, G304, G312, G314, G314D, G316, G320, G321, } \\
\text { G324 }\end{array}$ & ND \\
\hline Chloroform & $\begin{array}{l}\text { G300, G304, G312, G314, G314D, G316, G320, G321, } \\
\text { G324 }\end{array}$ & ND \\
\hline 1,2-Dichloroethane & $\begin{array}{l}\text { G300, G304, G312, G314, G314D, G316, G320, G321, } \\
\text { G324 }\end{array}$ & ND \\
\hline 2-Butanone & $\begin{array}{l}\text { G314 } \\
\text { G300, G304, G312, G314D, G316, G320, G321, G324 }\end{array}$ & 590 \\
\hline 1,1,1-Trichloroethane & $\begin{array}{l}\text { G300, G304, G312, G314, G314D, G316, G320, G321, } \\
\text { G324 }\end{array}$ & ND \\
\hline Carbon Tetrachloride & $\begin{array}{l}\text { G300, G304, G312, G314, G314D, G316, G320, G321, } \\
\text { G324 }\end{array}$ & ND \\
\hline Trichloroethene & $\begin{array}{l}\text { G300, G304, G312, G314, G314D, G316, G320, G321, } \\
\text { G324 }\end{array}$ & ND \\
\hline 1,1,2-Trichloroethane & $\begin{array}{l}\text { G300, G304, G312, G314, G314D, G316, G320, G321, } \\
\text { G324 }\end{array}$ & ND \\
\hline Benzene & $\begin{array}{l}\text { G300, G304, G312, G314, G314D, G316, G320, G321, } \\
\text { G324 }\end{array}$ & ND \\
\hline 4-Methyl-2-Pentanone & $\begin{array}{l}\text { G300, G304, G312, G314, G314D, G316, G320, G321, } \\
\text { G324 }\end{array}$ & ND \\
\hline
\end{tabular}




\begin{tabular}{|c|c|c|}
\hline Analyte & Sample Number & $\begin{array}{l}\text { Reported Value } \\
\quad(\mathrm{ug} / \mathrm{L})\end{array}$ \\
\hline Tetrachloroethene & $\begin{array}{l}\text { G300, G304, G312, G314, G314D, G316, G320, G321, } \\
\text { G324 }\end{array}$ & ND \\
\hline 1,1,2,2-Tetrachloroethane & G300, G304, G312, G314, G314D & ND \\
\hline Toluene & $\begin{array}{l}\text { G314 } \\
\text { G316 } \\
\text { G300, G304, G312, G314D, G320, G321, G324 }\end{array}$ & $\begin{array}{l}140^{*} \\
43^{*} \\
\text { ND }\end{array}$ \\
\hline Chlorobenzene & $\begin{array}{l}\text { G300, G304, G312, G314, G314D, G316, G320, G321, } \\
\text { G324 }\end{array}$ & ND \\
\hline Ethylbenzene & $\begin{array}{l}\text { G300, G304, G312, G314, G314D, G316, G320, G321, } \\
\text { G324 }\end{array}$ & ND \\
\hline $\mathrm{m}, \mathrm{p}$-Xylene & $\begin{array}{l}\text { G300, G304, G312, G314, G314D, G316, G320, G321, } \\
\text { G324 }\end{array}$ & ND \\
\hline 0 -Xylène & $\begin{array}{l}\text { G300, G304, G312, G314, G314D, G316, G320, G321, } \\
\text { G324 }\end{array}$ & ND \\
\hline Isopropylbenzene & $\begin{array}{l}\text { G300, G304, G312, G314, G314D, G316, G320, G321, } \\
\text { G324 }\end{array}$ & ND \\
\hline 1,3,5-Trimethylbenzene & $\begin{array}{l}\text { G300, G304, G312, G314, G314D, G316, G320, G321, } \\
\text { G324 }\end{array}$ & ND \\
\hline 1,2,4-Trimethylbenzene & $\begin{array}{l}\text { G300, G304, G312, G314, G314D, G316, G320, G321, } \\
\text { G324 }\end{array}$ & ND \\
\hline 1,2,3-Trimethylbenzene & $\begin{array}{l}\text { G300, G304, G312, G314, G314D, G316, G320, G321, } \\
\text { G324 }\end{array}$ & ND \\
\hline
\end{tabular}

ND $=$ Not Detected.

*Analyte identified below its CRQL.

There are no serious limitations or concerns regarding the information used to characterized this tank. However, a few assumptions were made:

- When density was not reported for a given sample, usually a heated one, the sample density was assumed as that of an unheated sample collected at the same position in the tank -- same riser, angle and distance from bottom of tank.

- $\quad$ All the data reported were used to obtain the maximum, minimum and average values. The data were reported as primary, duplicate, heated and unheated. Since the procedure used to analyze the data was not available to the technical reviewer, all the data were assumed to be relevant. Only when the analytical procedure becomes available can the reviewer establish if the primary and duplicate data should be averaged.

- Precipitation of sodium phosphate at ambient temperatures occurred for unheated samples -not maintained at the tank temperature, $40^{\circ} \mathrm{C}$. Many analytical measurements used acid 
digestion and the precipitate had little influence on the results, therefore unheated sample data was considered valid.

- The $\mathrm{pH}$ of the waste (13.7) was calculated based on from the reported hydroxide concentration. 


\subsection{AP105}

\subsection{Tank Summary}

Tank AP105 contains $583 \mathrm{~m}^{3}$ (154 kgal) of waste, composed entirely of saltcake solids (Hanlon 1996). In 1989, the tank was filled in two evaporator campaigns, which processed dilute noncomplexed waste into DSSF. In August 1995, the supernatant was transferred to AP101. After the transfer, in-tank photos revealed a solids layer had crystallized at the bottom of the tank. The solids were not recorded in the Waste Tank Summary Report until April 1996 (Hanlon 1996). The waste characterization data was collected and analyzed for the Privatization project prior to the transfer. The analysis may now be valid for the supernatant in AP101.

The composition of the tank contents prior to the August 1995 transfer is based on data obtained when the waste was sampled for the Grout Program in March, 1993 (Welsh 1994b). Twenty liquid grab samples were taken from four depths at each of three tank risers. Thirteen samples (one from each location and one duplicate) were analyzed for chemical species and radionuclides, and seven were analyzed for organic components.

Two composite samples were prepared from equal aliquots of the 12 individual samples - . representing each sampling location. The duplicate samples were not included in composite. Comparison of the means of analytical results showed no difference between individual and composite results.

\subsection{Reference Summary}

Table 9.1 summarizes the references from which the characterization data for waste in tank AP105 were retrieved. The summary includes the reference number from Kaiser's Master Set data, memo date, the reference citation, sample date and type, whether the data in the reference were accepted for use in the Subset data, and comments about the reference. Only one document (AP-105-2) contained valid data.

\subsection{Waste Volume and Sampling Events}

The waste volume and tank sampling event plot for AP105 is shown in Figure 9.1. Tank AP105 contains only solids because the supernatant was transferred to tank AP101 in 1995. Waste from tank AN101 is expected to be transferred into AP105 in August $1996^{(a)}$. Tank AP105 will then be used to feed evaporator campaigns 97-1 and 97-2. More transfers may .occur in conjunction with the tank's role as an evaporator feed tank.

\subsection{Master Set}

The Master Set originally contained four sources of data points. Most of the entries came

(a) Personal Communication with TM Blaak, 8/96. 
Table 9.1. Summary of Source Documents Containing Waste Characterization Data for AP105

\begin{tabular}{|c|c|c|c|c|c|c|}
\hline $\begin{array}{l}\text { Kaiser } \\
\text { Referenc } \\
\text { e } \\
\text { Number }\end{array}$ & $\begin{array}{c}\text { Memo } \\
\text { Date }\end{array}$ & Source Document* & $\begin{array}{c}\text { Sample } \\
\text { Date }\end{array}$ & $\begin{array}{c}\text { Sample } \\
\text { Type }\end{array}$ & $\begin{array}{c}\text { Accept/ } \\
\text { Reject }\end{array}$ & Comments \\
\hline AP-105-1 & $11 / 11 / 92$ & $\begin{array}{l}\text { Wilkins NE. 1992. Potential Addition of Tanks to } \\
\text { Watch List. Internal Memo \#7K210-92-457, Rev. } 1 \text { to } \\
\text { JC Fulton dated } 11 / 11 / 92 \text {, Westinghouse Hanford } \\
\text { Company, Richland, WA. }\end{array}$ & Unavailable & Supernatant & Reject & $\begin{array}{l}\text { Sample numbers: T-4317, T-4046, } \\
\text { T-3954, T-3789, T- } 3635 \text {. Grab } \\
\text { samples taken from evaporator slurry } \\
\text { line as tank was being filled. } \\
\text { Composition of evaporator product } \\
\text { varied during campaigns. Samples } \\
\text { taken in-tank are more reliable. }\end{array}$ \\
\hline AP-105-2 & $5 / 10 / 94$ & $\begin{array}{l}\text { Welsh TL. 1994. Tank 241-AP-105 Characterization } \\
\text { Results. WHC-SD-WM-TRP-169, Rev. 0, } \\
\text { Westinghouse Hanford Company, Richland, WA. }\end{array}$ & $3 / 93$ & Supernatant & Accept & $\begin{array}{l}14 \text { grab samples. The most } \\
\text { complete and reliable source of } \\
\text { information for tank AP105 waste }\end{array}$ \\
\hline
\end{tabular}

* Many source documents are not cleared for public release. They are cited to provide traceablility to the original data. 


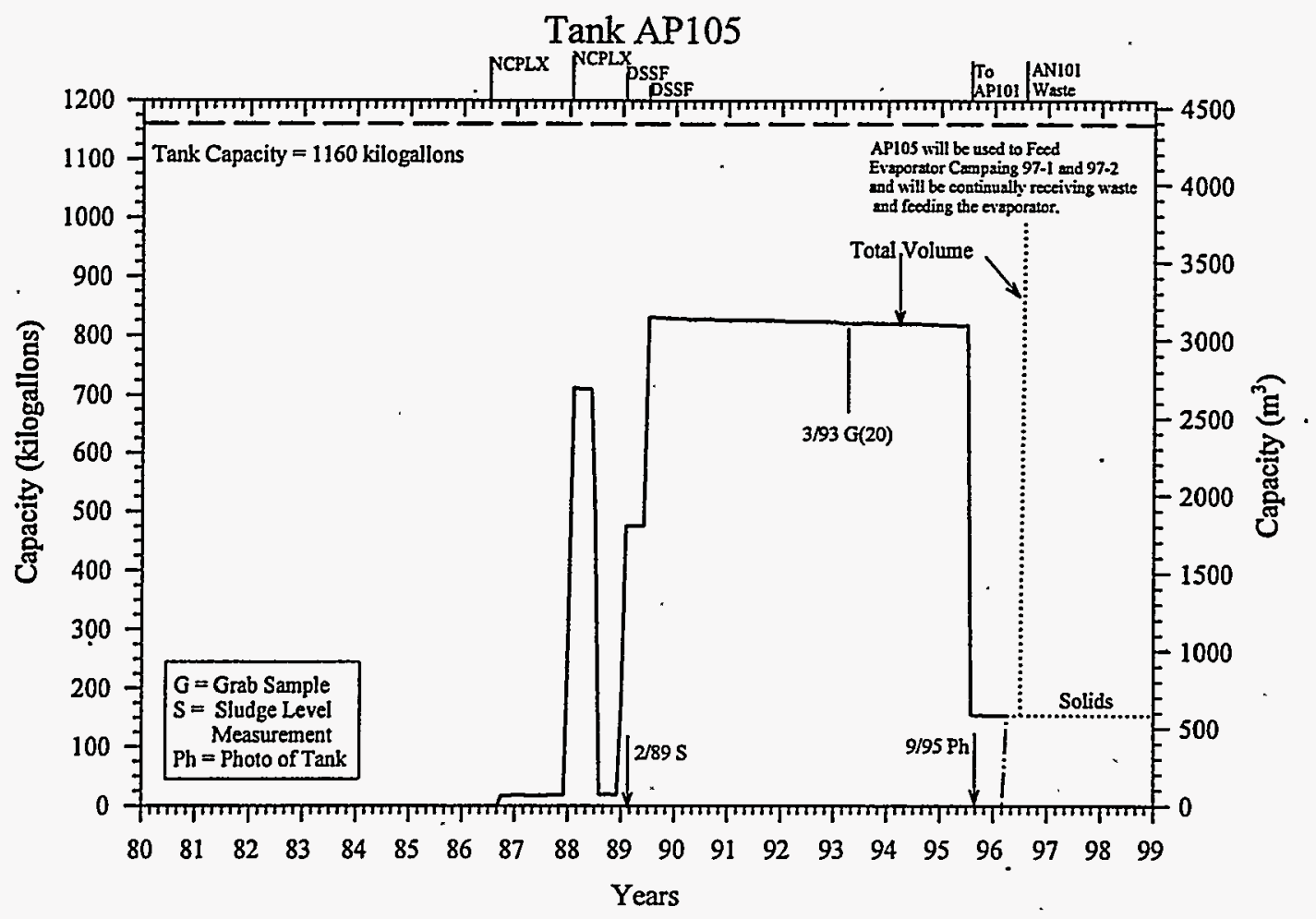

Figure 9.1. Tank Waste Volume and Sampling Event Graph for Tank AP105

from the TCD. Other sources were AP-105-2 (one or two entries for each analyte), the HTCE database (one entry for each analyte), and AP-105-1 (a few scattered entries).

The Master Set TCD data were derived from reference AP-105-2, which contained separate entries for primary and duplicate analyses for each sample. Because the laboratory quality assurance program requires that primary and duplicate samples agree within a specified range, the mean value for each sample was substitute for the primary and duplicate results. The source citation was changed from TCD to reference AP-105-2.

The statistical averages over all samples for each analyte and radionuclide as reported in AP-105-2 were included in the Master Set and identified in the "notes" column as statistical averages. These values were used for the "best estimate" concentrations.

Each point was reviewed by comparing the Master Set database entry to the original laboratory report to verify that the numbers and units were transcribed correctly and that the units were converted correctly to the standard $\mu \mathrm{g} / \mathrm{L}$ format.

\subsection{Subset}

Each point in the Master Set was reviewed to determine whether the analytical data 
correctly represented the current contents of the tank. For each valid point, a "Y" was inserted in the Master Set "Validated" column. For each invalid point, a " $\mathrm{N}$ " was inserted, and a comment was placed in the "Notes" column to indicate why the point was considered invalid. The "Subset" is composed of all of the " $Y$ " points in the Master Set.

In most cases, the Master Set points derived from reference AP-105-2 were identified as valid points, and the points derived from reference AP-105-1 were invalid. Most of the AP-105-2 points for fluoride were invalid because of the matrix interference discussed in the reference. The valid fluoride points were those run by a different method.

\section{6 $\mathrm{Min} / \mathrm{Max} / \mathrm{BE}$}

The lowest concentration in the Subset was selected as the minimum value; the highest concentration in the Subset was selected as the maximum value. The best estimate concentration was taken as the overall average of the results for the 13 individual and two composite samples from the 1993 assay (Welsh 1994b). The value used as the best estimate is in the Subset and is identified by the comments in the notes column.

\subsection{Organics}

Extensive analysis of organic constituents, including complexant, volatile, and semivolatile compound concentrations was conducted on seven samples obtained from AP105 in March 1993 (Welsh 1994b). The waste was analyzed for the following organic species:

Organic Constituents:

EDTA, HEDTA, Citrate, Oxalate, Glycolate, and

Semivolatile Species:

1-Butanol, Pyridine, 1,4-Dichlorobenzene1, 2-Dichlorobenzene, 2-Methylphenol, 4-Methylphenol, Hexachloroethane, Nitrobenzene, Hexachlorobutadiene, 2,4,6-Trichlorophenol, 2,4,5-Trichlorophenol, 2,4-Dinitrotoluene, Diethylphthalate, Tributylphosphate, Hexachlorobenzene, Pentachlorophenol, Di-n-octylphthalate, and

Volatile Species:

Vinyl Chloride, Methylene Chloride, Acetone, Carbon Disulfide, 1,1-Dichloroethene, Chloroform, 1,2-Dichloroethane, 2-Butanone, 1,1,1-Trichloroethane, Carbon Tetrachloride, Trichloroethene, 1,1,2-Trichloroethane, Benzene, 4-Methyl-2-Pentanone, Tetrachloroethene, 1,1,2,2-Tetrachloroethane, Toluene, Chlorobenzene, Ethylbenzene, m,p-Xylene, o-Xylene, Isopropylbenzene, 1,3,5-Trimethylbenzene, 1,2,4-Trimethylbenzene, 1,2,3-Trimethylbenzene

The waste contained EDTA (91 to $156 \mathrm{mg} / \mathrm{L}$ ), HEDTA (5 to $25 \mathrm{mg} / \mathrm{L}$ ), citrate (202 to 697 $\mathrm{mg} / \mathrm{L})$, glycolate $(500$ to $800 \mathrm{mg} / \mathrm{L})$, and oxalate $(120$ to $900 \mathrm{mg} / \mathrm{L})$. Acetone was identified in five of the seven samples ( 88 to $840 \mu \mathrm{g} / \mathrm{L}$ ). The value $88 \mu \mathrm{g} / \mathrm{L}$ is below the contract-required quantitation limit. No other volatile or semivolatile compound was detected in the waste samples. 


\subsection{Physical Properties}

The only physical property measurements were density $(1.34 \mathrm{~g} / \mathrm{mL}$ average $)$ and percent water (60.3\% average).

\subsection{Limitations/Concerns}

Grab samples were taken from 12 locations (from four depths under three risers) in the tank (plus one field duplicate sample). Comparisons among the 12 locations show that the supernate in the tank is clearly stratified, becoming more concentrated near the bottom. Therefore, if the waste were not mixed before waste retrieval, the composition would be expected to vary during the retrieval operation. The minimum and maximum concentration estimates provide some indication of the extent of inhomogeneity in the waste.

The calculated overall mass balance based on the best estimate, or average, 'concentrations of each of the waste components was $99.8 \%$, (ideal $=100 \%$ ). The calculated cation/anion charge balance was 0.97 (ideal $=1.00$ ). . The combination of the mass and charge balance results provides a high level of assurance that the data are representative of the waste in the tank prior to the August 1995 transfer. 


\subsection{AW101}

\subsection{Tank Summary}

Tank AW101 contains 4,266 $\mathrm{m}^{3}(1,127 \mathrm{kgal})$ of waste classified as DSSF (Hanlon 1996). The total volume is split between $3,948 \mathrm{~m}^{3}(1,043 \mathrm{kgal})$ of supernatant and $318 \mathrm{~m}^{3}$ (84 kgal) of sludge and floating crust (Welsh 1991). The tank is currently on the flammable tank watch list.

The tank received a large variety of waste additions during the early 1980 s, primarily PUREX miscellaneous wastes. In 1985, the tank was pumped down to a small volume of sludge $\left(204 \mathrm{~m}^{3} / 54 \mathrm{kgal}\right)$ and then filled with evaporator concentrate in 1986 . No waste has been transferred into or out of the tank since 1986, and no transfers are planned before the waste is sent to pretreatment.

The sludge layer was core sampled (two segments) in 1987. It consists of two fairly distinct layers. The bottom layer accumulated during the early 1980s, while the tank was receiving PUREX miscellaneous wastes. The upper layer of sludge and the floating crust are composed of salts formed from evaporator concentrate.

The supernatant layer was sampled extensively for the Grout Program in June 1990 (Welsh 1991). Twelve liquid grab samples were taken from four depths at each of three tank risers. The analytical results from those samples provide the current knowledge of the composition of the liquid in the tank.

\subsection{Reference Summary}

Table 10.1 summarizes the references from which the characterization data for waste in tank AW101 were retrieved. The summary includes the reference number from Kaiser's Master Set data, memo date, the source document citation, sample date and type, whether the data in the reference was accepted for use in the Subset data, and comments about the reference.

\subsection{Waste Volume and Sampling Events}

The waste volume and tank sampling event plot for AW101 is shown in Figure 10.1. The waste in the tank is DSSF from tank AW102, and the tank is near capacity. The total volume appears to be decreasing slightly due to evaporation.

The solids volume was last measured in October 1984 (Hanlon 1995), and no solids were found at that time. However, the solids volume increased suddenly in mid-1987. That increase was not accounted for initially. Discussion with Dan Herting indicated that the September 1987 core sample was taken from the heel in the tank before the DSSF was added and that the solids volume was measured at the same time. Because of the time lag in the Hanlon reports, the results were not recorded in the Hanlon reports until mid-1987. An in-tank photograph taken in March 1988 showed a layer of solids. The solids volume is projected to be constant but may be increasing slightly. 
Table 10.1. Summary of Source Documents Containing Waste Characterization Data for AW101

\begin{tabular}{|c|c|c|c|c|c|c|}
\hline $\begin{array}{l}\text { Kaiser } \\
\text { Reference } \\
\text { Number }\end{array}$ & $\begin{array}{c}\text { Memo } \\
\text { Date }\end{array}$ & Source Documents* & $\begin{array}{c}\text { Sample } \\
\text { Date }\end{array}$ & $\begin{array}{c}\text { Sample } \\
\text { Type }\end{array}$ & $\begin{array}{l}\text { Accept/ } \\
\text { Reject }\end{array}$ & Comments \\
\hline AW-101-1 & $12 / 30 / 81$ & $\begin{array}{l}\text { Maffeo R. 1981. Analysis of 101-AW BM's. } \\
\text { Internal memo \# 12510-81-368 and \#12510-81-369 } \\
\text { to MC Teats dated 12/30/81, Rockwell Hanford } \\
\text { Operations, Richland, WA. }\end{array}$ & Unavailable & Supernatant & Reject & $\begin{array}{l}\text { Grab samples, numbers T-1048 } \\
\text { and T-1049. Not used because } \\
\text { waste no longer in tank. }\end{array}$ \\
\hline$A W-101-2$ & $\begin{array}{l}11 / 09 / 84 \\
11 / 20 / 84\end{array}$ & $\begin{array}{l}\text { Mauss BM: 1984. Chemical Compositions of 102- } \\
\text { AY, 101-AW, 105-AN, and 104-AW. Internal memo } \\
\text { \# 65453-84-348 to EG Gratny dated 11/09/84, } \\
\text { Rockwell Hanford Operations, Richland, WA. and } \\
\text { Jansky MT. 1984. Chemical Composition of Tank } \\
101-A W \text { Waste. Internal memo \# 65453-84-356 to } \\
\text { DM Tulberg dated 11/20/84, Rockwell Hanford } \\
\text { Operations, Richland, WA. }\end{array}$ & NA & Supernatant & Reject & $\begin{array}{l}\text { Grab samples, numbers R-2644, } \\
\text { R-2645, and R-3185. Not used } \\
\text { because waste no longer in tank. }\end{array}$ \\
\hline AW-101-3 & $4 / 29 / 86$ & $\begin{array}{l}\text { Mauss BM. 1986. 86-3 Evaporator Campaign: } \\
\text { Laboratory Analysis Data. Internal memo \# } 65453- \\
86-060 \text { to RT Kumura dated } 4 / 29 / 86, \text { Rockwell } \\
\text { Hanford Operations, Richland, WA. }\end{array}$ & $\begin{array}{l}3 / 30 / 86 \\
\text { (analysis } \\
\text { date) }\end{array}$ & Supernatant & Reject & $\begin{array}{l}\text { Grab samples, numbers T- } 7697 \\
\text { and T-7698. Not used because } \\
\text { waste no longer in tank. }\end{array}$ \\
\hline AW-101-4 & $9 / 18 / 86$ & $\begin{array}{l}\text { Mauss BM. 1986. 86-5 Evaporator Campaign: } \\
\text { Laboratory Analysis of Samples. Internal memo \# } \\
65453-86-114 \text { to JC Starr dated } 9 / 18 / 86 \text {, Rockwell } \\
\text { Hanford Operations, Richland, WA. }\end{array}$ & $\begin{array}{l}6 / 23 / 86 \\
\text { through } \\
7 / 05 / 86\end{array}$ & Slurry & Accept & $\begin{array}{l}\text { Evaporator slurry samples, } \\
\text { numbers R- } 9893 \text { through R- } \\
9897 \text {. Results are included in } \\
\text { Subset, but are not as reliable as } \\
\text { samples direct from tank }\end{array}$ \\
\hline AW-101-5 & $7 / 21 / 87$ & $\begin{array}{l}\text { Weiss RL. 1987. Results of Analysis of Tank 241- } \\
\text { AW-10I Heel Samples. Internal memo \# } 65453-87- \\
105 \text { to WG Richmond dated 7/21/87, Westinghouse } \\
\text { Hanford Company, Richland, WA. }\end{array}$ & NA & Sludge & Reject & $\begin{array}{l}\text { Core sample of heel of sludge in } \\
\text { tank before it was filled with } \\
\text { DSSF. Not used because it was } \\
\text { not a liquid sample. }\end{array}$ \\
\hline AW-101-10 & $12 / 19 / 91$ & $\begin{array}{l}\text { Welsh TL. 1991. Tank } 241-A W-101 \\
\text { Characterization Test Results. WHC-SD-WM-TRP- } \\
\text { 055, Westinghouse Hanford.Company, Richland, } \\
\text { WA. }\end{array}$ & $6 / 28 / 90$ & Supernatant & Accept & $\begin{array}{l}\text { Sample numbers } R-4792 \text { through } \\
R-4803 \text {. Most complete and } \\
\text { reliable source of information } \\
\text { for the tank liquid. }\end{array}$ \\
\hline
\end{tabular}

* Many source documents are not cleared for public release. They are cited to provide traceablility to the original data. 


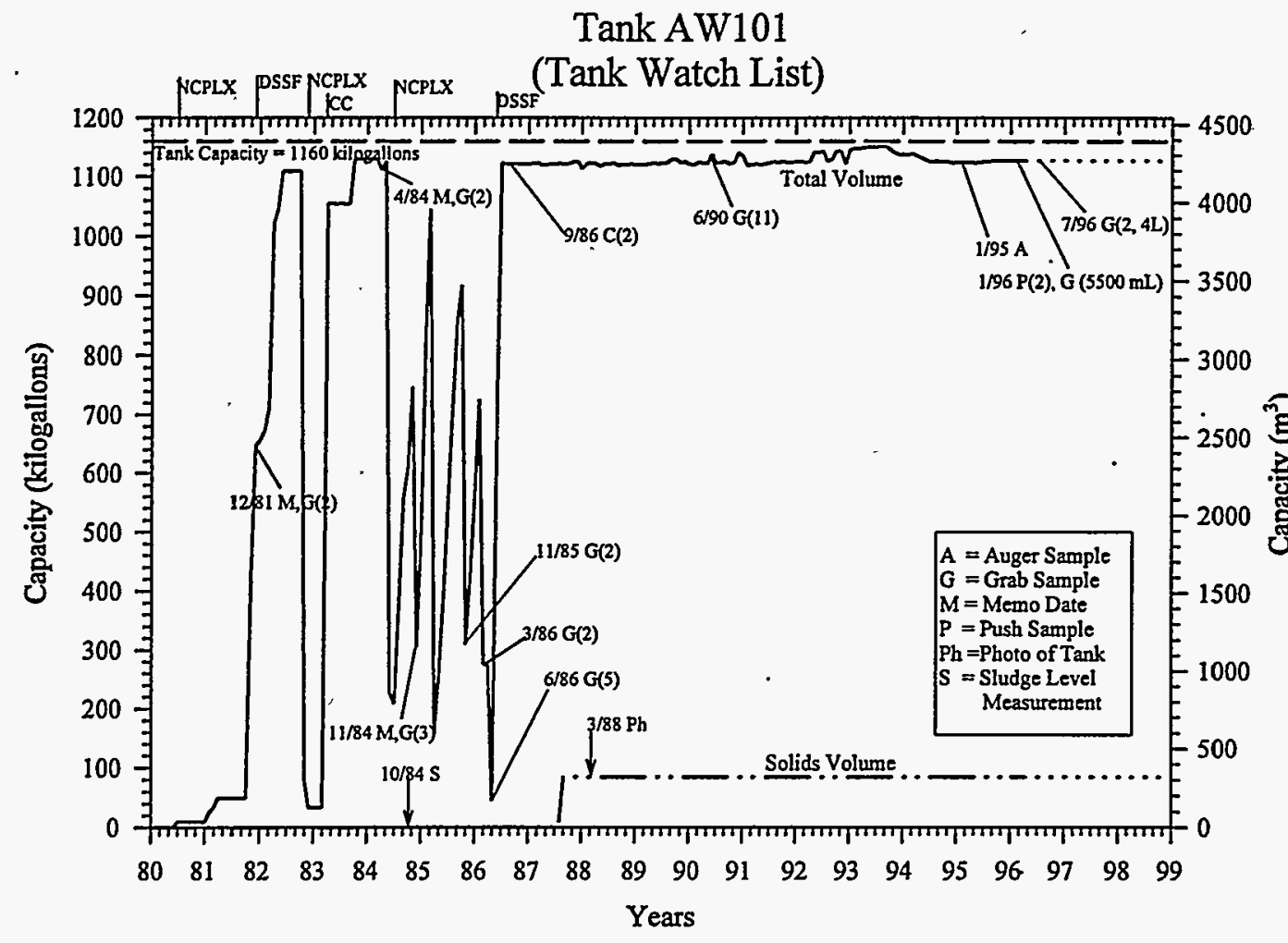

Figure 10.1. Tank Waste Volume and Sampling Event Graph for Tank AW101

No further transactions are planned for AW101 until the waste is sent to pretreatment.

\subsection{Master Set}

All of the analytical data from the Kaiser references were included in the Master Set. The evaluators compared the Master Set data was compared to the original laboratory report to verify that the numbers and units were transcribed correctly, and that the units were converted correctly to the standard $\mu \mathrm{g} / \mathrm{L}$ format. Dates and reference numbers were also checked for accuracy.

\subsection{Subset}

Each point in the Master Set was reviewed to determine whether the analytical data correctly represented the current contents of the liquid phase in the tank and points were disqualified in accordance with the criteria discussed in section 2.6. For each valid point, a "Y" was inserted in the Master Set "Validated" column. For each invalid point, a "N" was inserted, and a comment was placed in the "Notes" column of the Master Set to indicate why the point was considered invalid. The Subset is composed of all of the " $Y$ " points in the Master Set. 


\section{$10.6 \mathrm{Min} / \mathrm{Max} / \mathrm{BE}$}

The lowest and highest concentrations in the Subset were designated as the minimum and maximum, respectively. The best estimate value was taken as the average of the results for the two composite samples prepared from equal aliquots of the 12 individual samples from 1990 . Comparison of the composite and individual analytical results showed a consistent pattern of higher concentrations of the metals in the composite samples. The overall mass balance and charge balance are closer to $100 \%$ and 1.00 , respectively, for the composite samples than for the individual samples. Therefore, the composite results were used to define the "best estimate" concentrations for the tank liquid.

\subsection{Organics}

The total organic carbon (TOC) content of the composite was $2.46 \mathrm{~g} / \mathrm{L}$, below the $10 \mathrm{~g} / \mathrm{L}$ level that defines a "complexed waste". Therefore, the organic content is not expected to be a major factor in processing the waste for disposal. Screening for volatile organic compounds and semivolatile organic compounds was documented by Welsh (1991). Those results are questionable because volatile compounds detected in the waste sample were also detected in the method blank. The semivolatile organic analyses indicated that nitrobenzene and bis (2-ethylhexy) phthalate were detected but below the estimated quantitation limits. The compound tributyl phosphate (TBP) was not detected by the analysis, however based on the response of internal standard, it could be present in quantities of $50 \mathrm{ppm}$ or less. ${ }^{(a)}$

\subsection{Physical Properties}

Physical property measurements are limited to density $(1.56 \mathrm{~g} / \mathrm{mL}$ average $)$ and percent water (43.6\% average). The 1990 grab samples tended to form crystalline precipitates when samples cooled from tank temperature (approximately $42^{\circ} \mathrm{C}$ ) to room temperature in the laboratory. This behavior is expected when evaporator concentrates are saturated in one or more sodium salts.

\subsection{Limitations/Concerns}

Comparisons among the 12 grab samples taken from four depths under three risers show that the supernatant in the tank is fairly homogeneous. No transfers have been made in or out of the tank since these samples were taken.

The calculated overall mass balance based on the "best estimate" concentrations of each of the waste components was $99.6 \%$ (ideal $=100 \%$ ). The calculated cation/anion charge balance was 1.17 (ideal $=1.00$ ). The combination of the mass and charge balance results suggests that the cation components may be slightly overestimated, and the anions slightly underestimated.

(a) Personal Communication with $\mathrm{KD}$ Wiemers via ccmail 6/17/96. 


\subsection{AY102}

\subsection{Tank Summary}

Tank AY102 contains $2982 \mathrm{~m}^{3}$ (788 kgal) of dilute non-complexed (DN) waste (Hanlon 1996). The waste volume consists of $2861 \mathrm{~m}^{3}(756 \mathrm{kgal})$ supernatant and $121 \mathrm{~m}^{3}$ (32 kgal) sludge. The tank has received miscellaneous DN waste from B plant, T plant, 222-S and 300 Area laboratories, and other locations since the early 1980s. The tank is active.

\subsection{Reference Summary}

Table 11.1 summarizes the references from which the characterization data for waste in tank AY102 were retrieved. The summary includes the reference number from Kaiser's Master Set data, memo date, the source document citation, sample date and type, whether the data in the reference were accepted for use in the Subset data, and comments about the reference. Supernatant sampling results prior to 1994 do not accurately represent the tank composition because many transfers occurred during the previous 15 years. Supernatant composition and inventory are based on grab samples acquired in June and December 1994. Analyte concentrations in AY102 supernatant were obtained from the most recent tank characterization report (Ryan 1995a).

\subsection{Waste Volume and Sampling Events}

The waste volume and tank sampling event plot is shown in Figures 11.1 and 11.2. Dilute non-complexed waste has been transferred into and out of. Tank AY102 repeatedly since late 1981. During 1995, wastes from 222-S and 300 Area laboratories, B Plant, and T Plant were added to the tank. Approximately $280 \mathrm{kgal}\left(1060 \mathrm{~m}^{3}\right)$ of waste was transferred to tank AP102 in January 1996. It is predicted that in FY 2003, waste from C106 will be transferred to the tank and the supernatant will be transferred to AN101. Sọdium hydroxide and sodium nitrite solution will be added to the remaining sludge for corrosion control (Shelton 1996).

\subsection{Master Set/Subset}

Kaiser did not produce a Master Set for tank AY102 because it was not in the original scope of work. Likewise, no Subset was produced.

\subsection{Min/Max/BE}

The minimum and maximum values for a particular component were the lowest and highest analytical results, respectively, found in the TCR (Ryan 1995a). In a few cases, the minimum value corresponded to the analysis detection limit for that component (less than values). The best estimate was calculated as the average of the minimum and maximum values. 
Table 11.1. Summary of References Containing Waste Characterization Data for AY102

\begin{tabular}{|c|c|c|c|c|c|c|}
\hline $\begin{array}{c}\text { Kaiser } \\
\text { Reference } \\
\text { Number }\end{array}$ & $\begin{array}{c}\text { Memo } \\
\text { Date }\end{array}$ & Source Documents & $\begin{array}{c}\text { Sample } \\
\text { Date }\end{array}$ & $\begin{array}{c}\text { Sample } \\
\text { Type }\end{array}$ & $\begin{array}{l}\text { Accept/ } \\
\text { Reject }\end{array}$ & Comments \\
\hline$A Y-102-1$ & $6 / 25 / 74$ & $\begin{array}{l}\text { Wheeler RE. 1974. Tank Farm } \\
\text { Samples. Internal Letter to RL Walser } \\
\text { dated } 6 / 25 / 74 \text {, Atlantic Richfield Hanford } \\
\text { Operations, Richland, WA. }\end{array}$ & $\overline{\mathrm{NA}}$ & Supernatant & Reject & $\begin{array}{l}\text { Outdated document, (prior to last major } \\
\text { transfer). Limited information contained in } \\
\text { data table: pH, sodium concentration, and } \\
\text { Cs activity. }\end{array}$ \\
\hline AY-102-2 & $9 / 21 / 79$ & $\begin{array}{l}\text { Delegard CH. 1979. Analytical Data for } \\
\text { Tanks 101-AZ, 102-AZ, 103-TX, and 102- } \\
\text { AY Hot Synthetic Liquors. Internal Letter } \\
\# 65120-79-151 \text { J to LD Vanselow dated } \\
9 / 21 / 79, \text { Rockwell Hanford Operations, } \\
\text { Richland, WA. }\end{array}$ & $\overline{\mathrm{NA}}$ & Supernatant & Reject & $\begin{array}{l}\text { Outdated document, (prior to last major } \\
\text { transfer). Contains data for } 4 \text { supernatant } \\
\text { samples taken at two different levels from } \\
\text { two different risers prior to memo date. } \\
\text { Analytical results on chemical and } \\
\text { radionuclide content. }\end{array}$ \\
\hline$A Y-102-3$ & $4 / 15 / 81$ & $\begin{array}{l}\text { Jansky MT. 1981. Composition of } \\
\text { Waste From Tanks 101AY and } 102 A Y \text {. } \\
\text { Internal letter \# 65453-81-130 to MC } \\
\text { Teats dated 4/15/81, Rockwell Hanford } \\
\text { Operations, Richland, WA. }\end{array}$ & NA & $\begin{array}{l}\text { Slurry/ } \\
\text { Filtrate/ } \\
\text { Solids }\end{array}$ & Reject & $\begin{array}{l}\text { Outdated document, (prior to last major } \\
\text { transfer). Info on chemical composition and } \\
\text { radionuclides. Analytical data are } \\
\text { questionable. }\end{array}$ \\
\hline AY $-102-4$ & $6 / 18 / 82$ & $\begin{array}{l}\text { Panesko JV. 1982. Analysis of 102-AY } \\
\text { Supernate. Internal letter \# } 65453-082- \\
228 \text { to NW Kirch dated } 6 / 18 / 82 \text {, } \\
\text { Rockwell Hanford Operations, Richland, } \\
\text { WA. }\end{array}$ & $6 / 12 / 82$ & Supernatant & Reject & $\begin{array}{l}\text { Outdated document, (prior to last major } \\
\text { transfer). Lab support for upcoming } 242-\mathrm{A} \\
\text { evaporator campaign } 84-5 \text {. A surface } \\
\text { (R9833) and a } 4 \mathrm{ft} \text {. sample (R9834) were } \\
\text { analyzed. Contains analytical results on } \\
\text { chemical composition and radionuclides. }\end{array}$ \\
\hline AY-102-5 & $5 / 10 / 84$ & $\begin{array}{l}\text { Jansky MT. 1984. Laboratory Support } \\
\text { of.Upcoming 242-A Evaporator Campaign } \\
\text { Run } 85-4 \text {. Internal letter \# } 65453-84- \\
134 \text { to EG Gratny dated 5/10/84, } \\
\text { Rockwell Hanford Operations, Richland, } \\
\text { WA. }\end{array}$ & NA & Supernatant & Reject & $\begin{array}{l}\text { Outdated document, (prior to last major } \\
\text { transfer). Analytical results on a } 4 \mathrm{ft} \text {. } \\
\text { below the liquid surface sample were } \\
\text { analyzed. Contains chemical composition } \\
\text { and radionuclides. }\end{array}$ \\
\hline AY-102-6 & $11 / 9 / 84$ & $\begin{array}{l}\text { Mauss BM. 1984, Chemical } \\
\text { Compositions of 102-AY, 101-AW, 105- } \\
A N \text {, and 104- } A W . \text { Internal memo \# } \\
65453-84-348 \text { to EG Gratny dated } \\
11 / 9 / 84 \text {, Rockwell Hanford Operations, } \\
\text { Richland, WA }\end{array}$ & NA & Supernatant & Reject & $\begin{array}{l}\text { Outdated document, (prior to last major } \\
\text { transfer). Tank samples (R-3214 and R- } \\
3215 \text { ) from AY102 received by Chem. Lab } \\
\text { prior to memo date. Analytical results of } \\
\text { chemical and radionuclide composition and } \\
\text { physical properties on two supernatant } \\
\text { samples. }\end{array}$ \\
\hline
\end{tabular}




\begin{tabular}{|c|c|c|c|c|c|c|}
\hline $\begin{array}{l}\text { Kaiser } \\
\text { Reference } \\
\text { Number }\end{array}$ & $\begin{array}{c}\text { Memo } \\
\text { Date }\end{array}$ & Source Documents & $\begin{array}{l}\text { Sample } \\
\text { Date }\end{array}$ & $\begin{array}{l}\text { Sample } \\
\text { Type }\end{array}$ & $\begin{array}{c}\text { Accept/ } \\
\text { Reject }\end{array}$ & Comments \\
\hline AY-102-7 & $\overline{\mathrm{NA}}$ & $\begin{array}{l}\text { Certa PJ. 1985. 242-A Evaporator } \\
\text { Crystallizer FY } 84 \text { Campaign Run } 84-3 \\
\text { Post Run Document. SD-WM-PE-018, } \\
\text { Rockwell Hanford Operations, Richland, } \\
\text { WA. }\end{array}$ & $12 / 7 / 83$ & $\begin{array}{l}\text { Unslurped } \\
\text { Samples }\end{array}$ & $\overline{\text { Reject }}$ & $\begin{array}{l}\text { Outdated document, (prior to last major } \\
\text { transfer). Contains information on the } 242- \\
\text { A evaporator campaign } 84-3 \text {. Two } \\
\text { samples of AY102 processing feed (1st pass } \\
\text { samples) were analyzed for chemical } \\
\text { composition. }\end{array}$ \\
\hline$\overline{A Y-102-8}$ & $9 / 30 / 88$ & $\begin{array}{l}\text { Scott PA. 1989. Results of the } \\
\text { Characterization of 102-AY Waste Solids, } \\
\text { Washed Solids, and Supernate. Letter } \\
\text { Report for Milestone 0603-A to AJ } \\
\text { DiLiberto dated 9/30/88, Pacific } \\
\text { Northwest Laboratory, Richland, WA. }\end{array}$ & $\overline{N A}$ & Core & Reject & $\begin{array}{l}\text { Outdated document, (prior to last major } \\
\text { transfer). Additional characterization results } \\
\text { on samples AY102 Seg-1, AY } 102 \text { Seg } 1 \mathrm{R} \\
\text { Top, AY102 Seg } 1 \mathrm{R} \text { Mid, and AY102 Seg } \\
\text { 1R Bot. Report contains results of physical, } \\
\text { chemical, and radionuclide analyses. }\end{array}$ \\
\hline AY-102-9 & $1 / 29 / 88$ & $\begin{array}{l}\text { Scott PA. 1988. TRU Content in 102- } \\
\text { AY Tank Waste Solids. Letter Report to } \\
\text { AJ DiLiberto dated 1/29/88, Pacific } \\
\text { Northwest Laboratory, Richland, WA. }\end{array}$ & NA & Solids & Reject & $\begin{array}{l}\text { Outdated document, (prior to last major } \\
\text { transfer). Characterization results on } \\
\text { samples AY102 Seg 1R Top, AY102 Seg } \\
\text { IR Mid, and AY102 Seg 1R Bot. Contains } \\
\text { information on the TRU content, density, } \\
\text { and \%water of a core sample (Reference to } \\
\text { lab book BNW 51353). The core was split } \\
\text { into } 4 \text { segments. A composite was made } \\
\text { from the lower three segments and } \\
\text { analyzed. The top (supernatant) section was } \\
\text { archived. }\end{array}$ \\
\hline AY-102-10 & $1 / 19 / 89$ & $\begin{array}{l}\text { Peterson ME. 1989. Revised Tables for } \\
\text { I02-AY Characterizations. Letter report } \\
\text { to LM Sasaki dated } 1 / 19 / 89, \text { Pacific } \\
\text { Northwest Laboratory, Richland, WA. }\end{array}$ & $\overline{\mathrm{NA}}$ & $\begin{array}{l}\text { Solids/ } \\
\text { Supernatant }\end{array}$ & Reject & $\begin{array}{l}\text { Correction to AY-102-8. Outdated } \\
\text { document,(data prior to last major transfer). } \\
\text { Corrected data tables for previously issued } \\
\text { report. }\end{array}$ \\
\hline$A Y-102-11$ & $4 / 21 / 89$ & $\begin{array}{l}\text { Womack JC. 1989. Tank 102-AY Solids } \\
\text { Heel Analysis. DSI to HF Daugherty } \\
\text { dated 4/21/89, Westinghouse Hanford } \\
\text { Company, Richland, WA. }\end{array}$ & NA. & Solids & Reject & Request for reanalysis on FY88 results. \\
\hline
\end{tabular}




\begin{tabular}{|c|c|c|c|c|c|c|}
\hline $\begin{array}{c}\text { Kaiser } \\
\text { Reference } \\
\text { Number }\end{array}$ & $\begin{array}{l}\text { Memo } \\
\text { Date }\end{array}$ & Source Documents & $\begin{array}{l}\text { Sample } \\
\text { Date }\end{array}$ & $\begin{array}{l}\text { Sample } \\
\text { Type }\end{array}$ & $\begin{array}{c}\text { Accept/ } \\
\text { Reject }\end{array}$ & Comments \\
\hline$\overline{A Y-102-12}$ & $2 / 12 / 90$ & $\begin{array}{l}\text { Peterson ME. 1990. Revised Report on } \\
\text { the Results of } 102-A Y \text { Characterization. } \\
\text { Letter Report \#9000855 to AJ DiLiberto } \\
\text { dated } 2 / 12 / 90 \text {, Pacific Northwest } \\
\text { Laboratory, Richland, WA. }\end{array}$ & $\overline{\mathrm{NA}}$ & $\begin{array}{l}\text { Solids/ } \\
\text { Supernatant }\end{array}$ & Reject & $\begin{array}{l}\text { Correction to AY-102-8. Outdated } \\
\text { document, (data prior to last major } \\
\text { transfer). Revised report contains summary } \\
\text { of characterization on samples received from } \\
\text { WHC FY88-2 in Q2 FY1988 (sample \#s: } \\
\text { AY102 Seg 1R Top, AY102 Seg 1R Mid, } \\
\text { and AY102 Seg IR Bot). }\end{array}$ \\
\hline AY-102-13 & $7 / 21 / 88$ & Analysis Report $1 / 29 / 88$ & $\overline{\mathrm{NA}}$ & Solids & Reject & $\begin{array}{l}\text { Reference to AY-102-9. Outdated } \\
\text { document, (data prior to last major } \\
\text { transfer). Analysis date reported as } \\
1 / 29 / 88 \text {. Contains only data tables on } \\
\text { physical properties, radionuclides, and } \\
\text { chemicals without additional information. }\end{array}$ \\
\hline AY-102-14 & $11 / 6 / 90$ & Analysis Report $2 / 12 / 90$ & $\overline{\mathrm{NA}}$ & Solids & Reject & $\begin{array}{l}\text { Reference to AY-102-12. Outdated } \\
\text { document, (data prior to last major } \\
\text { transfer). Analysis date reported as } \\
2 / 12 / 90 \text {. Analyses of composite sample } \\
\text { \#AY-102. Contains only data tables on } \\
\text { physical properties, radionuclides, and } \\
\text { chemicals without additional information. }\end{array}$ \\
\hline AY-102-15 & $11 / 6 / 90$ & Analysis Report $2 / 12 / 90$ & $\overline{N A}$ & Solids & Reject & $\begin{array}{l}\text { Reference to AY-102-12. Outdated } \\
\text { document, (data prior to last major } \\
\text { transfer). Analysis date reported as } \\
2 / 12 / 90 \text {. Rheological characterization of } \\
\text { diluted composite sample \#AY-102D }(1: 1 \\
\text { with deionized water). Contains only data } \\
\text { tables without additional information. }\end{array}$ \\
\hline AY-102-16 & $11 / 6 / 90$ & Analysis Report $2 / 12 / 90$ & Supernatant & & Reject & $\begin{array}{l}\text { Reference to AY-102-12. Outdated } \\
\text { document, (data prior to last major } \\
\text { transfer). Analysis date reported as } \\
2 / 12 / 90 \text {. Analyses of composite sample } \\
\text { \#AY-1021. Contains only data tables on } \\
\text { radionuclides and chemical analytes without } \\
\text { additional information. }\end{array}$ \\
\hline
\end{tabular}




\begin{tabular}{|c|c|c|c|c|c|c|}
\hline $\begin{array}{c}\text { Kaiser } \\
\text { Reference } \\
\text { Number } \\
\end{array}$ & $\begin{array}{c}\text { Memo } \\
\text { Date }\end{array}$ & Source Documents & $\begin{array}{c}\text { Sample } \\
\text { Date }\end{array}$ & $\begin{array}{l}\text { Sample } \\
\text { Type }\end{array}$ & $\begin{array}{c}\text { Accept/ } \\
\text { Reject }\end{array}$ & Comments \\
\hline$\overline{\mathrm{AY}-102-17}$ & $11 / 6 / 90$ & Analysis Report $2 / 12 / 90$ & $\mathrm{NA}$ & Solids & $\overline{\text { Reject }}$ & $\begin{array}{l}\text { Reference to AY-102-12. Outdated } \\
\text { document, (data prior to last major } \\
\text { transfer). Analysis date reported as } \\
2 / 12 / 90 \text {. Analyses of composite sample } \\
\text { \#AY-1022. Contains only data tables on } \\
\text { physical properties, radionuclides, and } \\
\text { chemicals without additional information. }\end{array}$ \\
\hline AY-102-18 & $8 / 9 / 93$ & $\begin{array}{l}\text { Castaing BA. 1993. 101-AY, 102-AY, } \\
\text { and 106-C Data Compendium. WHC-SD- } \\
\text { WM-TI-578, Rev. 0, Westinghouse } \\
\text { Hanford Company, Richland, WA. }\end{array}$ & NA & $\begin{array}{l}\text { Supernatant } \\
\text { / Solids }\end{array}$ & Reject & $\begin{array}{l}\text { AY-102-6, AY-102-9, AY-102-12. } \\
\text { Outdated document,(data prior to last major } \\
\text { transfer). Compilation of data from lab } \\
\text { reports dating back to } 1975 \text {. }\end{array}$ \\
\hline AY-102-19 & $7 / 95$ & $\begin{array}{l}\text { Ryan GW. 1995. Tank Characterization } \\
\text { Report for Double-shelled Tank 241-AY- } \\
\text { 102. WHC-SD-WM-ER-454, Rev. 0, } \\
\text { Westinghouse Hanford Company, } \\
\text { Richland, WA. }\end{array}$ & $\begin{array}{l}6 / 8 / 94 \\
\text { and } \\
12 / 6 / 94\end{array}$ & Supernatant & Accept & $\begin{array}{l}\text { First citing of data. Supernatant } \\
\text { composition (physical properties, chemical } \\
\text { and radionuclide composition) reported for } \\
\text { June and Dec. } 1994 \text { grab samples. Also } \\
\text { contains data of June } 1987 \text { core sample. } \\
\text { Refers to an archived core sample from a } \\
12 / 94 \text { sampling event and has not been } \\
\text { characterized. }\end{array}$ \\
\hline
\end{tabular}




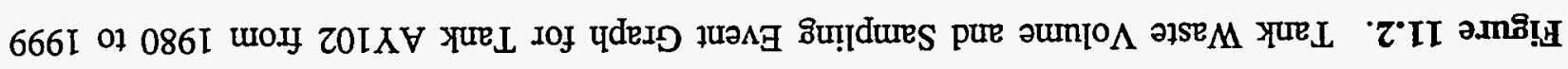

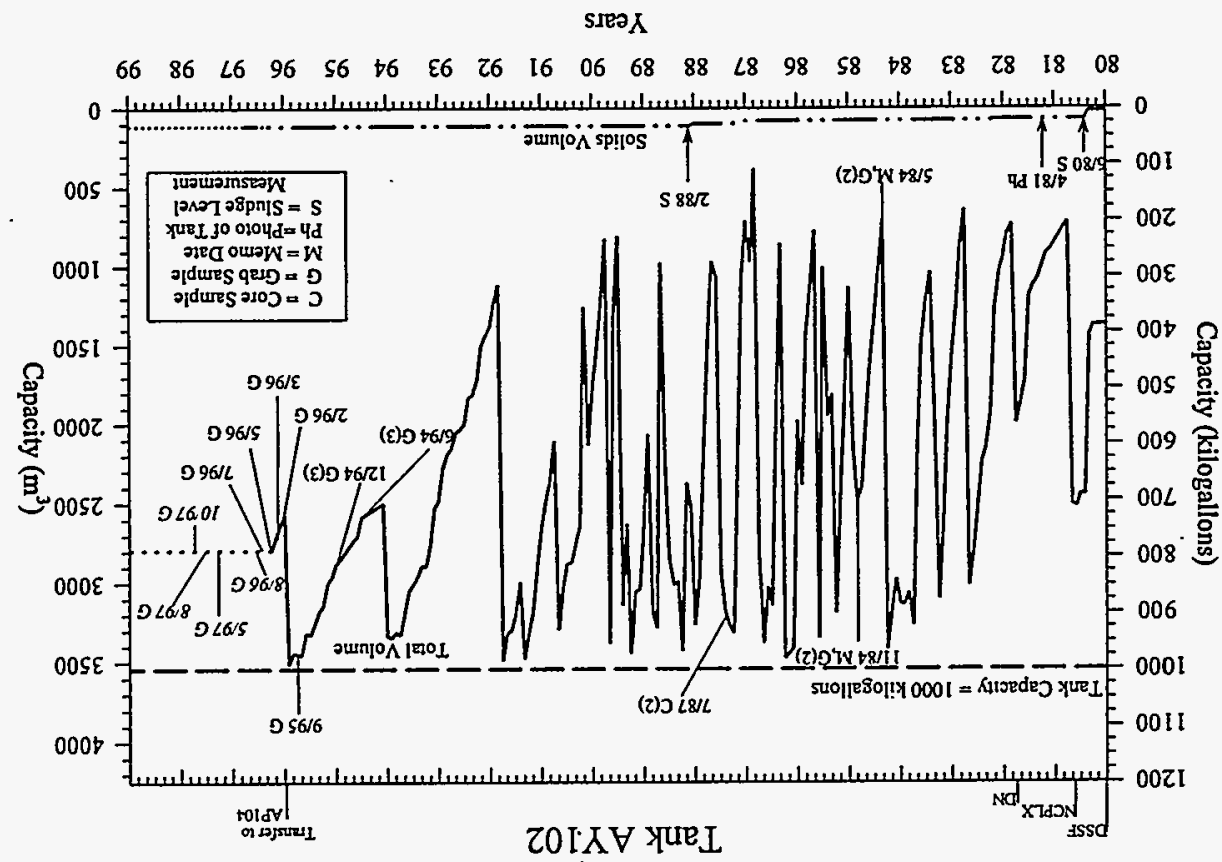

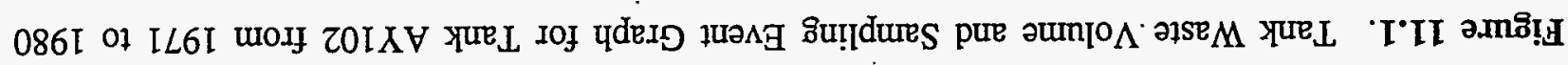

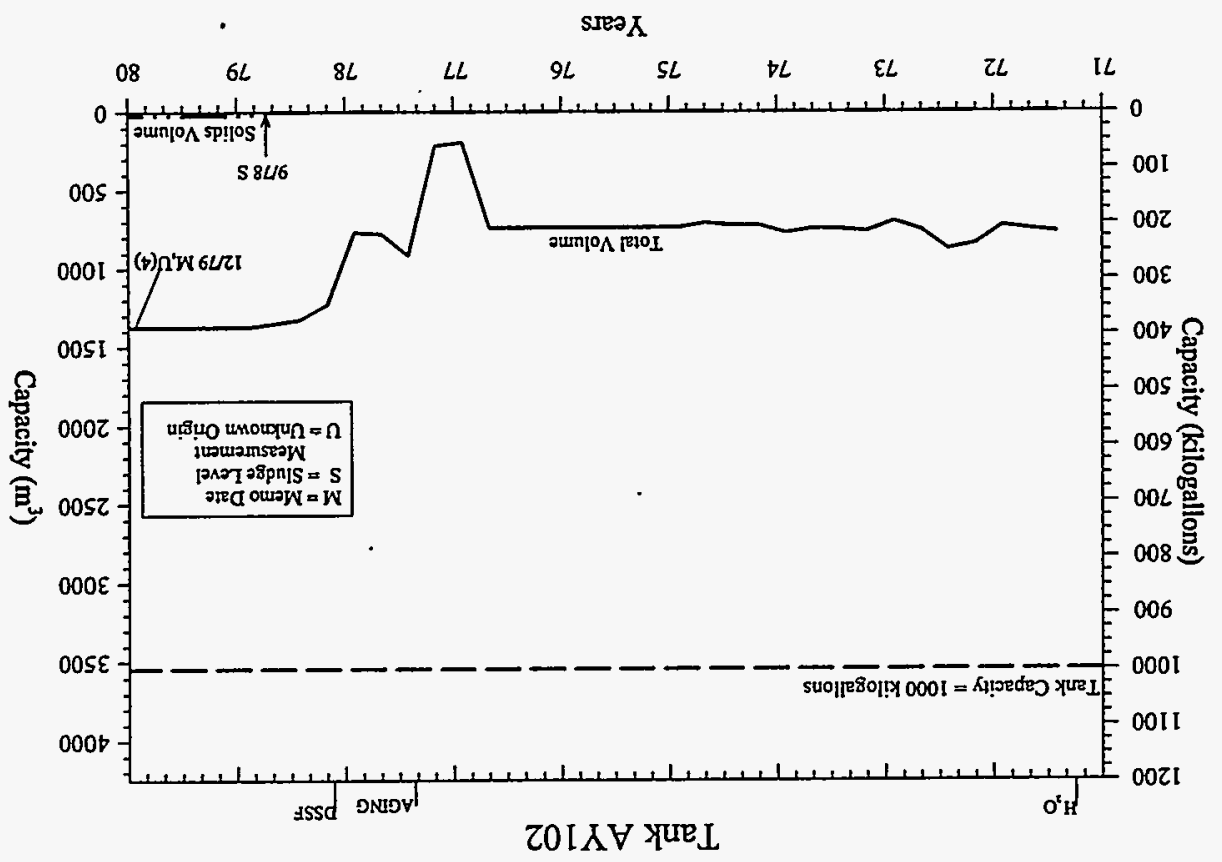




\subsection{Organics}

The waste composition of AY102 was based on the most recent tank characterization report (Ryan 1995a). Total organic carbon in the supernatant ranged from $7.68 \mathrm{E}-02$ to $8.87 \mathrm{E}-02 \mathrm{~g} / \mathrm{L}$. This report contained limited data on the organic components of the supernatant. Analysis was limited to oxalate, which was present in the December 1994 supernatant grab samples at a concentration of $5 \times 10^{-4} \mathrm{M}$.

\subsection{Physical Properties}

Few data are available on physical properties of the supernatant (Ryan 1995a). Grab samples of supernatant were taken June 8 and December 6,1994 . The supernatant samples were described as clear, yellow solutions containing $98.5 \%$ water and $<1 \%$ solids. The supernatant is DN waste with a density of $0.99 \mathrm{~g} / \mathrm{mL}$ and $\mathrm{pH} 11.3$.

\subsection{Limitations/Concerns}

The most recent sampling events were used to estimate the tank supernatant composition because previous results no longer represent the contents of the tanks. Five major transfers made since 1987 have changed the tank volume by $\sim 70 \%$ to $80 \%$. The supernatant composition of the tank waste is based on grab samples of supernatant taken in June and December 1994. The waste composition of AY102 was based on the most recent tank characterization report (Ryan 1995a), which contained data for all analytes. Limited analyses were. performed on cation, anion, and radionuclide analytes. Waste compatibility sample results (June 1994) were the average of the primary and duplicate samples. Process sample results (December 1994) were not analyzed in duplicate and were limited to anions and TOC concentrations.

Sludge samples were taken in December 1994 and archived for future analysis. The last core sample analyzed was taken in 1987, and these results no longer represent the solids composition of the tank. An estimate of the solids composition could be obtained from the December 1994 sludge grab sample.

Inventory estimates from the HTCE (Brevick, Gaddis, and Pickett 1995) of the concentration of chemical and radiological components were compared with 1994 analytical results from supernatant samples. Inventory results were higher than measured values by at least an order of magnitude. 


\subsection{AZ101}

\subsection{Tank Summary}

Tank AZ101 contains $3357 \mathrm{~m}^{3}$ (887 kgal) of waste, including $132 \mathrm{~m}^{3}$ (35 kgal) of solids designated as neutralized current acid waste (NCAW) (Hanlon 1996). The tank received evaporator waste, DSSF, non-complexed waste, water, residual liquor, and CC waste from 1976 until 1983. In August of 1983 the tank was emptied and then received NCAW from September 1983 until March 1986. Subsequent transfers have been water and condensate from AY and AZ farms.

\subsection{Reference Summary}

Table 12.1 summarizes the references from which the characterization data for waste in tank AZ101 were retrieved. The summary includes the reference number from Kaiser's Master Set data, memo date, the source document citation, sample date and type, whether the data in the reference were accepted for use in the Subset data, and comments about the reference.

\subsection{Waste Volume and Sampling Events}

The waste volume and tank sampling event plot is shown in Figure 12.1. The tank began service in the fourth quarter of 1976 when it started receiving evaporator waste (Q4 1976 - Q4 1977). From the first quarter of 1978 until September 1980, the tank received complexed waste, DSSF waste, non-complexed waste, water, evaporator waste, residual liquor and CC waste. The tank received non-complexed waste from October 1980 until January 1984. ${ }^{\text {(a) }}$ In August 1983, the tank was emptied of waste except for a heel of $\sim 757 \mathrm{~m}^{3}$ (20 kgal). From September 1983 until March $1986,-3028 \mathrm{~m}^{3}(800 \mathrm{kgal})$ of aging waste was transferred into the tank. In June 1989 , $568 \mathrm{~m}^{3}$ (150 kgal) of water was added to the tank. This may be an accumulation of many smaller transfers with a total volume of $568 \mathrm{~m}^{3}$. The 13 transfers into AZ101 made since January 1992 consisted mainly of condensate (water) collected from AY and AZ farms in the 702-A ventilation system.

The supernatant from this tank is to be decanted to tank AY101 in FY 1997. The tank solids will be washed twice in FY98 and the wash water sent to the evaporator. Sodium hydroxide and sodium nitrate solution will be added to the solids for corrosion control

The stepped appearance of solids volume plot is an artifact of measurement intervals. The solids volume in AZ101 was last measured in September 1990.

(a) There appears to be some discrepancy between references about the time frames for transfers in and out of some of the waste types. 
Table 12.1. Summary of Source Documents Containing Waste Characterization Data for AZ101

\begin{tabular}{|c|c|c|c|c|c|c|}
\hline $\begin{array}{l}\text { Kaiser } \\
\text { Reference } \\
\text { Number }\end{array}$ & $\begin{array}{c}\text { Memo } \\
\text { Date }\end{array}$ & Source Documents* & $\begin{array}{l}\text { Sample } \\
\text { Date }\end{array}$ & $\begin{array}{l}\text { Sample } \\
\text { Type }\end{array}$ & $\begin{array}{l}\text { Accept/ } \\
\text { Reject }\end{array}$ & Comments \\
\hline$A Z-101-1$ & $4 / 26 / 78$ & $\begin{array}{l}\text { Buckingham JS. 1978. Evaporation of } \\
\text { Complexed Waste in Tanks } 101-A Z \text { and } 102- \\
\text { AZ. Internal Letter \# } 60120-78-011 \mathrm{~J} \text { to KG } \\
\text { Carothers dated } 4 / 26 / 78 \text {, Rockwell Hanford } \\
\text { Operations, Richland, WA. }\end{array}$ & Unavailable & & Reject & Pre-Q3 1984. \\
\hline$A Z-101-2$ & $12 / 3 / 79$ & $\begin{array}{l}\text { Delegard CH. 1979. Relative Random Error } \\
\text { Standard Deviation and Accuracy in Hanford } \\
\text { Waste Liquor Analytical Data. Internal memo } \\
\text { \# 65124-79-029 to JS Buckingham et al. dated } \\
\text { 12/3/79, Rockwell Hanford Operations, } \\
\text { Richland, WA. }\end{array}$ & Unavailable & $\begin{array}{l}\text { Waste } \\
\text { Liquor }\end{array}$ & Reject & Pre-Q3 1984. \\
\hline$A Z-101-3$ & $8 / 27 / 81$ & $\begin{array}{l}\text { Jansky MT. 1981. Composition.of Waste } \\
\text { from Tanks 101-AZ and 102-AZ. Internal memo } \\
\text { \# 65453-81-283 to MC Teats dated } 8 / 27 / 81 \text {, } \\
\text { Rockwell Hanford Operations, Richland, WA. }\end{array}$ & Unavailable & Supernatant & Reject & Pre-Q3 1984. \\
\hline AZ-101-4 & $12 / 28 / 83$ & $\begin{array}{l}\text { Jansky MT. 1983. Tank 101AZ Sludge. } \\
\text { Internal memo \# 65453-83-311 to TM Kady } \\
\text { dated 12/28/83, Rockwell Hanford Operations, } \\
\text { Richland, WA. }\end{array}$ & Unavailable & Centrate & Reject & $\begin{array}{l}\text { Pre-Q3 1984. Sample contained solids, } \\
\text { sample } 3 \text { R7788. }\end{array}$ \\
\hline AZ-101-5 & $4 / 6 / 84$ & $\begin{array}{l}\text { Jansky MT. 1984. Organic Content of Tank } \\
\text { lOI-AZ Sludge. Internal Letter \# } 65453-84-103 \\
\text { to RB Bendixsen et al. dated } 4 / 6 / 84 \text {, Rockwell } \\
\text { Hanford Operations, Richland, WA. }\end{array}$ & Unavailable & Centrate & Reject & $\begin{array}{l}\text { Pre-Q3 1984. Corrects TOC reported in } \\
\text { AZ-101-4. }\end{array}$ \\
\hline AZ-101-6 & $9 / 10 / 84$ & $\begin{array}{l}\text { Jansky MT. 1984. Composition of Tank } \\
\text { 101AZ Sample Analysis. Internal letter \# } \\
65453-84-303 \text { to DJ Swanberg dated } 9 / 10 / 84 \text {, } \\
\text { Rockwell Hanford Operations, Richland, WA. }\end{array}$ & $6 / 84$ & Supernatant & Accept & $\begin{array}{l}\text { Table } 1 \text { contains data on some cations, } \\
\text { anions, } \mathrm{Cs}, \mathrm{Sr}, \mathrm{Pu} .\end{array}$ \\
\hline
\end{tabular}




\begin{tabular}{|c|c|c|c|c|c|c|}
\hline $\begin{array}{l}\text { Kaiser } \\
\text { Reference } \\
\text { Number }\end{array}$ & $\begin{array}{c}\text { Memo } \\
\text { Date }\end{array}$ & Source Documents* & $\begin{array}{c}\text { Sample } \\
\text { Date }\end{array}$ & $\begin{array}{c}\text { Sample } \\
\text { Type }\end{array}$ & $\begin{array}{l}\text { Accept/ } \\
\text { Reject }\end{array}$ & Comments \\
\hline AZ-10i-7 & $9 / 13 / 85$ & $\begin{array}{l}\text { Jansky MT. 1986. Composition of Tank 101- } \\
\text { AZ Neutralized Current Acid Waste. Internal } \\
\text { letter \#65453-86-003 to AJ DiLiberto dated } \\
\text { 1/10/86, Rockwell Hanford Operations, } \\
\text { Richland, WA. }\end{array}$ & Unavailable & Supernatant & Accept & $\begin{array}{l}\text { Table } 1 \text { gives Csi37 data by GEA. Table } 3 \\
\text { lists data for cations, anions, Cs, Sr, Tc, U, } \\
\text { density, } \mathrm{pH} \text {. }\end{array}$ \\
\hline AZ-101-8 & $1 / 10 / 86$ & $\begin{array}{l}\text { Jansky MT. 1986. Composition of Tank 101- } \\
\text { AZ Neutralized Current Acid Waste. Internal } \\
\text { Letter \# 65453-86-003 to AS DiLiberto dated } \\
1 / 10 / 86 \text {, Rockwell Hanford Operations, } \\
\text { Richland, WA. }\end{array}$ & Ụnavailable & Supernatant & Accept & $\begin{array}{l}\text { Tables } 1,2,3 \text { gives data for } \mathrm{Cs} \text {, anions, } \\
\text { cations, U, Sr, Tc. }\end{array}$ \\
\hline AZ-101-9 & $4 / 8 / 86$ & $\begin{array}{l}\text { Sasaki LM. 1986. '101-AZ NCAW Analysis } \\
\text { Evaluation. Internal memo \# 65611-86-039 } \\
\text { to AJ DiLiberto dated } 4 / 8 / 86, \text { Rockwell } \\
\text { Hanford Operations, Richland, WA. }\end{array}$ & $11 / 85$ & Supernatant & Reject & Evaluation of earlier data. (AZ-101-7) \\
\hline$A Z-101-10$ & $9 / 22 / 86$ & $\begin{array}{l}\text { Sasaki LM. 1986. NCAW TRU Analysis. } \\
\text { DSI to NW Kirch dated 9/22/86, Westinghouse } \\
\text { Hanford Company, Richland, WA. }\end{array}$ & Unavailable & Supernatant & Accept & Value for Am-241 only. \\
\hline AZ-101-11 & $12 / 4 / 86$ & $\begin{array}{l}\text { Sasaki LM. 1986. TRU Analysis of NCAW } \\
\text { from Tank 101-AZ. DSI to DR Bratzel, AJ } \\
\text { DiLiberto et al. dated 12/4/86, Westinghouse } \\
\text { Hanford Company, Richland, WA. }\end{array}$ & Unavailable & Supernatant & Accept & Data for actinides. \\
\hline$A Z-101-12$ & $1 / 28 / 87$ & $\begin{array}{l}\text { Mauss BM. 1987. Supernatant Analysis of } \\
101-\text { AZ Aging Waste. Internal memo \# } \\
65453-87-013 \text { to TG Hanson dated } 1 / 28 / 87 \text {, } \\
\text { Rockwell Hanford Operations, Richland, WA. }\end{array}$ & Unavailable & Supernatant & Accept & $\begin{array}{l}\text { Sample \# R9964 \& R0247. Samples } \\
\text { combined, gives anions, cations, pH, } \\
\text { density, Cs-137 }\end{array}$ \\
\hline
\end{tabular}




\begin{tabular}{|c|c|c|c|c|c|c|}
\hline $\begin{array}{l}\text { Kaiser } \\
\text { Reference } \\
\text { Number }\end{array}$ & $\begin{array}{c}\text { Memo } \\
\text { Date }\end{array}$ & Source Documents* & $\begin{array}{l}\text { Sample } \\
\text { Date }\end{array}$ & $\begin{array}{l}\text { Sample } \\
\text { Type }\end{array}$ & $\begin{array}{l}\text { Accept/ } \\
\text { Reject }\end{array}$ & Comments \\
\hline AZ-101-14 & $9 / 10 / 87$ & $\begin{array}{l}\text { Bausano JA. 1987. Solid/Liquid Phase } \\
\text { Distribution of Radionuclides in Aging Waste. } \\
\text { Internal Memo \# 65611-87-144 to RM } \\
\text { Marusich dated 9/10/87, Westinghouse Hanford } \\
\text { Company, Richland, WA. }\end{array}$ & Unavailable & Supernatant & Reject & $\begin{array}{l}\text { Comparisons of data from previous reports } \\
\text { (AZ-101-8 and AP-101-9) with expectations } \\
\text { from Origin code. }\end{array}$ \\
\hline$A Z-101-15$ & $3 / 11 / 88$ & $\begin{array}{l}\text { Herting, DL. 1988. Chemical Analysis of } \\
\text { Tank 101-AZ and 102-AZ Samples Taken in } \\
\text { October 1987. Internal memo \# 12221-PCL88- } \\
\text { 091 to LA Mihalik dated 3/11/88, } \\
\text { Westinghouse Hanford Company, Richland, } \\
\text { WA. }\end{array}$ & $11 / 87$ & Supernatant & Reject & Data is contained in AZ-101-16 \\
\hline AZ-101-16 & $2 / 8 / 89$ & $\begin{array}{l}\text { Hohl TM. 1989. Recent Sample Analyses } \\
\text { From Tanks } A Z 101, A Z 102 \text { and SY } 102 \text {. DSI } \\
\text { to SJ Skurla dated } 2 / 8 / 89 \text {, Westinghouse } \\
\text { Hanford Company, Richland, WA. }\end{array}$ & $11 / 87$ & Supernatant & Accept & $\begin{array}{l}\text { Sample \# T-3493, T-3494. Contains data on } \\
\text { cations, anions, TOC, U, Am, Sr, Tc \& Cs. }\end{array}$ \\
\hline$A Z-101-17$ & $2 / 5 / 90$ & $\begin{array}{l}\text { Jensen L. 1990. Characterization of Waste in } \\
241-A Z-101 \text { and } 102 \text {. Internal Memo \# 12711- } \\
90-016 \text { to DJ Flesher dated 2/5/90, } \\
\text { Westinghouse Hanford Company, Richland, } \\
\text { WA. }\end{array}$ & Unavailable & Supernatant & Reject & Comparison of various earlier samplings. \\
\hline$A Z-101-18$ & $2 / 28 / 90$ & $\begin{array}{l}\text { Peterson ME. 1990. Preliminary Results - } \\
\text { Analysis of Waste Layers from DST-101-AZ } \\
\text { Core \#2). Letter \#9001040 to AJ DiLiberto } \\
\text { and LM Sasaki dated } 2 / 28 / 90 \text {, Pacific } \\
\text { Northwest Laboratory, Richland, WA. }\end{array}$ & $4,5 / 89$ & Centrate & Reject & Preliminary report. \\
\hline AZ-101-19 & 9/93 & $\begin{array}{l}\text { Gray WJ, ME Peterson, RD Scheele, and JM } \\
\text { Tingey. 1993. Characterization of the Second } \\
\text { Core Sample of Neutralized Current Acid Waste } \\
\text { from Double-shelled Tank 101-AZ. Pacific } \\
\text { Northwest Laboratory, Richland, WA. }\end{array}$ & $4,5 / 89$ & Supernatant & Accept & $\begin{array}{l}\text { Data for cations (Table 9), anions (Table } \\
\text { 10), \& radionuclides (Table 11). }\end{array}$ \\
\hline
\end{tabular}




\begin{tabular}{|c|c|c|c|c|c|c|}
\hline $\begin{array}{l}\text { Kaiser } \\
\text { Reference } \\
\text { Number }\end{array}$ & $\begin{array}{c}\text { Memo } \\
\text { Date }\end{array}$ & Source Documents* & $\begin{array}{l}\text { Sample } \\
\text { Date }\end{array}$ & $\begin{array}{l}\text { Sample } \\
\text { Type }\end{array}$ & $\begin{array}{l}\text { Accept/ } \\
\text { Reject }\end{array}$ & Comments \\
\hline AZ-101-21 & $10 / 29 / 90$ & Report analysis dated $10 / 29 / 90$ & Unavailable. & Supernatant & Accept & $\begin{array}{l}2 \text { different samples?, Sample \# R-280187 } \\
\text { \&R101283, Data for Al, Cr, Na, OH, SO4 } \\
\text { \& Cs137, TOC. }\end{array}$ \\
\hline AZ-101-22 & $10 / 30 / 90$ & Report analysis dated 10/30/90. & Unavailable & Supernatant & Accept & $\begin{array}{l}\text { Sample \# R-110388, data for Al, Cr, Na, } \\
\text { SO4, TOC, OH \& Cs-137. }\end{array}$ \\
\hline$A Z-101-23$ & $2 / 11 / 91$ & $\begin{array}{l}\text { McGrail BP. 1991. Results of the Analysis of } \\
\text { the Large Chunk of Material and the } \\
\text { Measurement of the Miller Number for DST } \\
101-A Z \text { Core \#3 Waste. PNL letter \#9101055 } \\
\text { to LM Sasaki dated 2/11/91, Pacific Northwest } \\
\text { Laboratory, Richland, WA. }\end{array}$ & Unavailable & $\begin{array}{l}\text { Large piece } \\
\text { of solid } \\
\text { material }\end{array}$ & Reject & Analysis of solids. \\
\hline$A Z-101-24$ & $9 / 29 / 89$ & $\begin{array}{l}\text { Peterson ME. 1989. Final Report - } \\
\text { Characterization of the First Core Sample of } \\
\text { Neutralized Current Acid Waste from Double- } \\
\text { shelled Tank 101-AZ. Letter to AJ DiLiberto } \\
\text { dated 9/29/89, Pacific Northwest Laboratory, } \\
\text { Richland, WA. }\end{array}$ & FY89 & Supernatant & Accept & $\begin{array}{l}\text { Data for cations, anions, TOC, \& } \\
\text { radionuclides is given in Tables } 2,3,4,7 \\
\& 8 \text {. }\end{array}$ \\
\hline \multirow[t]{2}{*}{ AZ-101-25 } & $7 / 30 / 92$ & $\begin{array}{l}\text { Knapton TJ. } 1992 . \text { Radionuclide } \\
\text { Concentration Data for Tanks 106-C, 105-C, } \\
\text { and 101-AZ. Internal Memo \# 92-7K220-035 } \\
\text { to JC Van Keuren dated } 7 / 30 / 92 \text {, } \\
\text { Westinghouse Hanford Company, Richland, } \\
\text { WA. }\end{array}$ & Unavailable & Supernatant & Reject & $\begin{array}{l}\text { Comparison of radionuclide content for } \\
\text { various tanks using several different } \\
\text { sampling events. See AZ-101-24 }\end{array}$ \\
\hline & $7 / 26 / 95$ & $\begin{array}{l}\text { Hodgson KM. 1995. Tank Characterization } \\
\text { Report for Double-shelled Tank 24I-AZ-101. } \\
\text { WHC-SD-WM-ER-410, Westinghouse Hanford } \\
\text { Company, Richland, WA. }\end{array}$ & $4,5 / 89$ & Supernatant & Accept & $\begin{array}{l}\text { TCR, Appendix B contains data } \\
\text { for this sampling event, data } \\
\text { for Al, Fe, Na, anions, Am, Cs, } \\
\text { Pu isotopes, Sr, w\%H2O, } \\
\text { density, pH, TOC, TIC. Also } \\
\text { gives data from } 1989 \text { and } 1987 \\
\text { sampling events. }\end{array}$ \\
\hline
\end{tabular}

* Many source documents are not cleared for public release. They are cited to provide traceablility to the original data. 


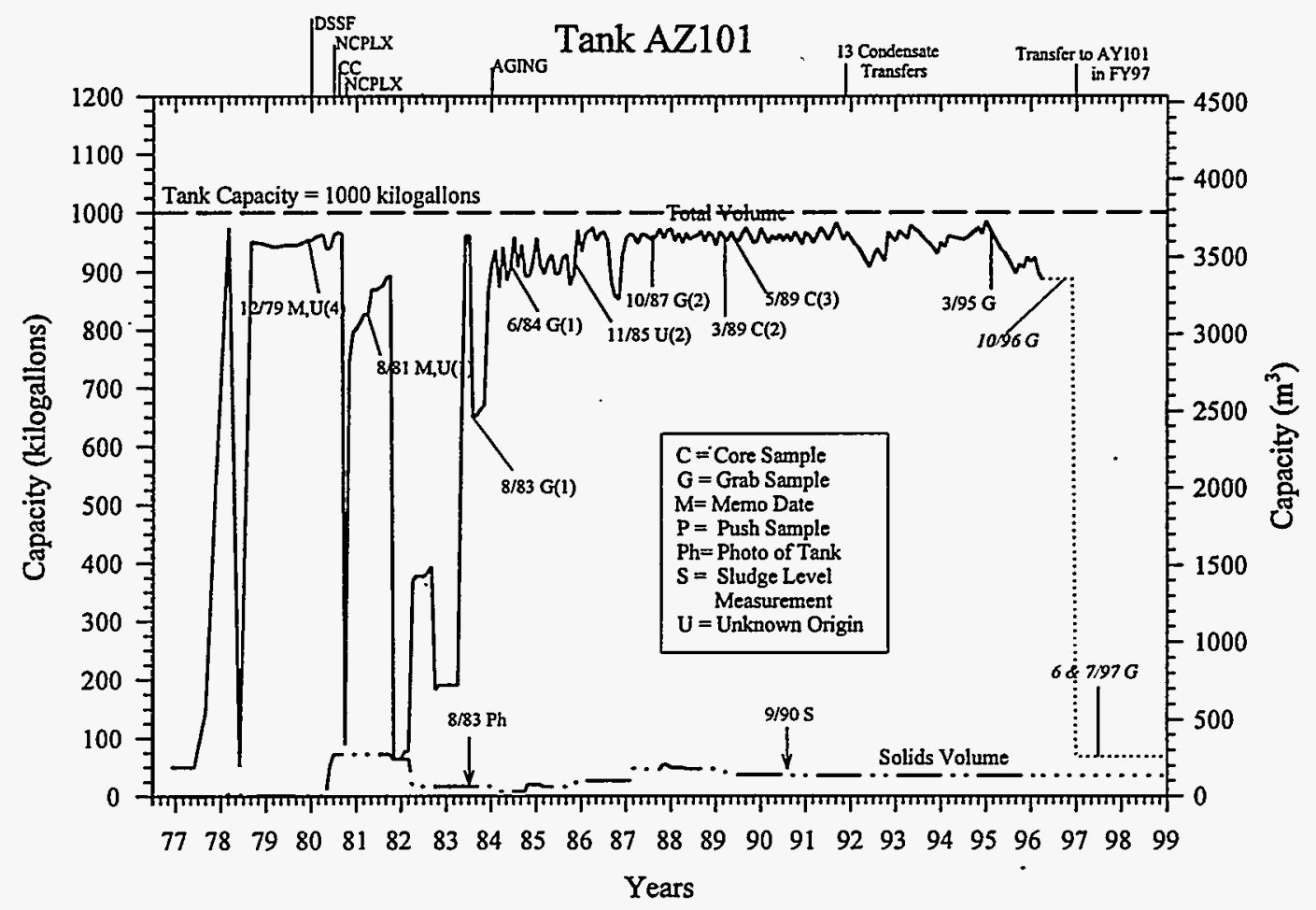

Figure 12.1. Tank Waste Volume and Sampling Event Graph for AZ101

\subsection{Master Set}

All of the analytical data from the Kaiser references were included in the Master Set. The Master Set data entry was compared to the original laboratory report to verify that the numbers and units were transcribed correctly, and that the units were converted correctly to the standard $\mu \mathrm{g} / \mathrm{L}$ format. Dates and reference numbers were also checked for accuracy.

\subsection{Subset}

Of the 26 documents in the Master Set, 12 were used to validate the Subset. Five documents published before September 1984 were not used because transfers had changed the waste composition.

All data from TRAC, Braun or Wastren were discarded. The HTCE data were kept but not factored into the $\mathrm{min} / \mathrm{max} /$ best estimate. Likewise "less than" values were kept but not used for the $\mathrm{min} / \mathrm{max} /$ best estimate, unless they were the only data for a species, as was the case for Fe in the TCR were "less than" values. If duplicate samples had been taken as in the TCR, the values for each sample were averaged; otherwise different sample aliquots were kept as individual analyses. If one of the values was a "less than" value and the other not, both values were 
included in the Subset.

\subsection{Min/Max/BE}

Only data from 1989 (core samples) and later were used for the $\min / \mathrm{max} / \mathrm{best}$ estimate. The TCR (Hodgson 1995) released in mid 1995 uses the same data which indicates that the data is most representative of the current tank contents.

If data from March 1995 were available, they were used exclusively to determine minimum, maximum, and best estimate. The best estimate was the average of the March 1995 sample data. If March 1995 data for a particular species were not available, the 1989 core sample data were used. The only exception was potassium, which was not measured in the March 1995 samples or the 1989 core samples. Data from a previous sampling were used to determine the minimum, maximum, and best estimate of potassium levels.

\subsection{Organics}

Although a large number of analyses have been done on this tank the organic species (acetate, formate, oxalate) have never been measured in either the supernatant or the sludge. The most recent report of the amount of TOC and TIC is in the TCR (Hodgson 1995).

\subsection{Physical Properties}

In 1984, $\sim 5$ vol\% solids was measured in supernatant samples (AZ-101-6). In 1985 supernatant samples were described as having dark brown, mud-like settled solids with clear supernatant and no suspended solids (AZ-101-7).

Additional data on percent solids (3-5 vol\%) and the effect of water washes and various element solubilities are presented in a January 1986 memo from Jansky (AZ-101-7). The solids were described as dark.

In April 1986, two samples obtained in December 1985 by the bottle-on-a-string were found to contain about 9 vol\% solids. From these analyses, the NCAW in the tank is estimated to contain about $20 \%$ solids (AZ-101-9).

In March 1988, one sample of supernatant and one sample of sludge each were taken from tanks AZ101 and AZ102. The AZ101 samples contained clear, bright yellow liquids with a trace of brown solids (AZ-101-15).

Core \#1 was analyzed and a report including physical and chemical properties was issued in September 1989. These core samples were taken when major transfers into and out of this tank were at a minimum. The supernatant from this core had a density of $1.2 \mathrm{~g} / \mathrm{mL}$, a $\mathrm{pH}=13.7$, and $26.9 \mathrm{wt} \%$ dissolved solids (AZ-101-24).

Physical property measurements on Core \#2, including weight percent solids and mean particle size, were reported in a letter dated February 1990 (AZ-101-18). More complete data on this core are in an October 1990 letter, and a final report was issued in 1993 (AZ-101-19). 
Miller number measurements on core \#3 are in a February 1991 report (AZ-101-23).

The TCR (Hodgson 1995) for tank AZ101 includes both physical property measurements and chemical/radionuclide analyses; however no data on the physical observations of the samples are available.

\subsection{Limitations/Concerns}

This tank was well characterized. Comparison of the data from Cores 1 and 2 with the TCR data should support the validity of the data for this tank. The best estimate tables for tank AZ101 were generated from the TCR data except for the minor constituents. The more recent data from the TCR should reflect the current tank contents.

A comparison of TCR values for waste in AZ101 and AZ102 showed that the concentrations of certain species are dissimilar. These tanks contain NCAW wastes generated from PUREX at approximately the same time so that it is reasonable to assume that the wastes are similar. It is difficult to explain the order of magnitude (or greater) discrepancies for all radionuclides except cesium-137 and strontium-90. Although the data in the published AZ101 TCR appear to be correct, the TCRs for AZ101 and AZ102 have been through a number of revisions, which makes one skeptical of any and all of the data in the valid Subset. 


\subsection{AZ102}

\subsection{Tank Summary}

Tank AZ102 entered service in 1976 and has a total storage capacity of $3785 \mathrm{~m}^{3}(1,000$ kgal). Its designation as a dilute receiver (DRCVR) tank means that the composition of the supernatant may change in the future (Hanlon 1996). As of April 1996, its the inventory was $3565 \mathrm{~m}^{3}$ (942 kgal) of NCAW, consisting $3205 \mathrm{~m}^{3}(847 \mathrm{kgal})$ supernatant and $360 \mathrm{~m}^{3}$ (95 kgal) sludge. The NCAW is designated as aging waste and was generated in the first cycle of the Plutonium Uranium Extraction (PUREX) process during its last campaign. The activity levels of fission products are relatively high in this tank.

\subsection{Reference Summary}

Table 13.1 summarizes the references from which the characterization data for waste in tank AZ102 were retrieved. The summary includes the reference number from Kaiser's Master Set data, memo date, the source document citation, sample date and type, whether the data in the reference was accepted for use in the Subset data, and comments about the reference.

\subsection{Waste Volume and Sampling Events}

The waste volume and tank sampling event plot is shown in Figure 13.1. Since January 1992, the tank has received 23 condensate transfers into the tank and some dilute non-complexed waste transfers from PUREX and B Plant. The supernatant in tank AZ102 will be concentrated in tank and decanted to tank AY101 in FY 2000 (Shelton 1996). The tank solids will be washed twice in FY 2000 and the wash water sent to the evaporator. Sodium hydroxide and sodium nitrate solution will be added for corrosion control.

\subsection{Master Set}

All of the analytical data from the Kaiser references were included in the Master Set. The Master Set data entry was compared to the original laboratory report to verify that the numbers and units were transcribed correctly, and that the units were converted. correctly to the standard $\mu \mathrm{g} / \mathrm{L}$ format. Dates and reference numbers were also checked for accuracy.

\subsection{Subset}

Each point in the Master Set was reviewed to determine whether the analytical data correctly represented the current contents of the liquid phase in the tank and invalid points were disqualified in accordance with the criteria discussed in section 2.6. For each valid point, a "Y" was inserted in the Master Set "Validated" column. For each invalid point, a "N" was inserted, and a comment was placed in the "Notes" column of the Master Set to indicate why the point was considered invalid. The Subset is composed of all of the "Y" points in the Master Set. Of 24 references reviewed, only two contained valid and current data (AZ-102-23 and AZ-102-24). The data from these reference were used to generate the Subset. 
Table 13.1. Summary of Source Documents Containing Waste Characterization Data for AZ102

\begin{tabular}{|c|c|c|c|c|c|c|}
\hline $\begin{array}{l}\text { Kaiser } \\
\text { Reference } \\
\text { Number }\end{array}$ & $\begin{array}{c}\text { Memo } \\
\text { Date }\end{array}$ & Source Documents* & $\begin{array}{l}\text { Sample } \\
\text { Date }\end{array}$ & Sample Type & $\begin{array}{l}\text { Accept/ } \\
\text { Reject }\end{array}$ & Comments \\
\hline AZ-102-1 & $4 / 26 / 78$ & $\begin{array}{l}\text { Buckingham JS. 1978. Evaporation of } \\
\text { Complexed Waste in Tank 102-AZ. Internal } \\
\text { Letter \# 65120-78-011J to KG Carothers } \\
\text { dated 4/26/78, Rockwell Hanford } \\
\text { Operations, Richland, WA. }\end{array}$ & Unavailable & Supernatant & Reject & $\begin{array}{l}\text { Tank contained } 2717 \mathrm{~m}^{3}(718 \mathrm{kgal}) \text { of } \\
\text { complexed waste. Outdated analysis. }\end{array}$ \\
\hline AZ-102-1 & $4 / 27 / 78$ & $\begin{array}{l}\text { Buckingham JS. 1978. Viscosity and } \\
\text { Cooling Curve of Concentrated Synthetic } \\
\text { 102-AZ Solutions. Internal Letter \# } 65120- \\
\text { 78-013J to KG Carothers dated } 4 / 27 / 78 \text {, } \\
\text { Rockwell Hanford Operations, Richland, } \\
\text { WA. }\end{array}$ & Unavailable & Supernatant & Reject & $\begin{array}{l}\text { Additional data from AZ-102-1. Outdated } \\
\text { analysis. }\end{array}$ \\
\hline AZ-102-3 & $12 / 3 / 79$ & $\begin{array}{l}\text { Delegard CH. 1979. Relative Random } \\
\text { Error, Standard Deviation, and Accuracy in } \\
\text { Hanford Waste Liquor Analytical Data. } \\
\text { Internal Letter \# } 65124-79-029 \text { to JS } \\
\text { Buckingham et al. dated } 12 / 3 / 79 \text {, Rockwell } \\
\text { Hanford Operations, Richland, WA. }\end{array}$ & Unavailable & Supernatant & Reject & $\begin{array}{l}\text { Four supernatant samples from two different } \\
\text { risers, both chemicals and radionuclides } \\
\text { analyzed. Outdated analysis. }\end{array}$ \\
\hline AZ-102-4 & $8 / 27 / 81$ & $\begin{array}{l}\text { Jansky MT. 1981. Composition of Waste } \\
\text { from Tanks } 101-A Z \text { and } 102-A Z \text {. Internal } \\
\text { memo \# 65453-81-283 to MC Teats dated } \\
\text { 8/27/81, Rockwell Hanford Operations, } \\
\text { Richland, WA. }\end{array}$ & Unavailable & $\begin{array}{l}\text { Supernatant/ } \\
\text { Suspended } \\
\text { Solids }\end{array}$ & Reject & $\begin{array}{l}\text { One sample analyzed for chemicals and } \\
\text { radionuclides. Tank contains complexed } \\
\text { concentrate. Outdated analysis. }\end{array}$ \\
\hline AZ-102-5 & $7 / 26 / 83$ & $\begin{array}{l}\text { Buckingham JS. 1983. Characterization of } \\
102 A Z \text { Waste Tank. Internal memo \# } \\
65453-83-191 \text { to TM Kady dated } 7 / 26 / 83 \text {, } \\
\text { Rockwell Hanford Operations, Richland, } \\
\text { WA. }\end{array}$ & Unavailable & $\begin{array}{l}\text { Supernatant/ } \\
\text { Suspended } \\
\text { Solids }\end{array}$ & Reject & $\begin{array}{l}\text { One sample analyzed to verify tank } \\
\text { composition before emptying contents. Both } \\
\text { chemicals and radionuclides analyzed. } \\
\text { Outdated analysis. }\end{array}$ \\
\hline
\end{tabular}




\begin{tabular}{|c|c|c|c|c|c|c|}
\hline $\begin{array}{l}\text { Kaiser } \\
\text { Reference } \\
\text { Number }\end{array}$ & $\begin{array}{l}\text { Memo } \\
\text { Date }\end{array}$ & Source Documents* & $\begin{array}{l}\text { Sample } \\
\text { Date }\end{array}$ & Sample Type & $\begin{array}{l}\text { Accept } \\
\text { Reject }\end{array}$ & Comments \\
\hline$A Z-102-6$ & $8 / 19 / 83$ & $\begin{array}{l}\text { Buckingham JS. 1983. Analysis of TK } \\
102 A Z \text { Supernatant Liquid. Internal Letter } \\
\# 65453-83-213 \text { to RB Bendixson et al. } \\
\text { dated 8/19/83, Rockwell Hanford } \\
\text { Operations, Richland, WA. }\end{array}$ & Unavailable & & Reject & $\begin{array}{l}\text { This document is a summary of } 4 \text { prior } \\
\text { analyses. Two of the original references } \\
\text { reported here are missing one from } 6 / 26 / 79 \\
\text { and one from } 9 / 20 / 82 \text {. Data contained in } \\
\text { other references (AZ-102-4 and AZ-102-5) }\end{array}$ \\
\hline$A Z-102-7 a$ & 9/1/83 & $\begin{array}{l}\text { Jansky MT. 1983. Corrected Radionuclide } \\
\text { Content of Tank } 102 A Z \text { Sludge. Internal } \\
\text { Letter \# 65453-83-228 to TM Kady et al. } \\
\text { dated 9/1/83, Rockwell Hanford } \\
\text { Operations, Richland, WA. }\end{array}$ & Unavailable & Sludge & Reject & $\begin{array}{l}\text { Contains corrections to the radionuclide } \\
\text { concentrations in AZ-102-7b. Data } \\
\text { contained in other references. }\end{array}$ \\
\hline AZ-102-7b & $8 / 26 / 83$ & $\begin{array}{l}\text { Jansky MT. 1983. 102AZ Sludge Sample. } \\
\text { Internal memo \# 65453-83-221 to TM Kady } \\
\text { dated August 26. Rockwell Hanford } \\
\text { Operations, Richland, WA. }\end{array}$ & Unavailable & Sludge & Reject & $\begin{array}{l}\text { May be original data but not clear contains } \\
\text { information on the acid and water soluble } \\
\text { portions of sludge samples. Outdated } \\
\text { analysis. }\end{array}$ \\
\hline AZ-102-8 & $2 / 4 / 85$ & $\begin{array}{l}\text { Bratzel DR. 1985. Tank 102-AZ Chemical } \\
\text { Analysis Results, R3699 and R3700. } \\
\text { Internal memo \# } 65453-85-026 \text { to DM } \\
\text { Tulberg dated 2/4/85, Rockwell Hanford } \\
\text { Operations, Richland, WA. }\end{array}$ & Unavailable & Supernatant & Reject & $\begin{array}{l}\text { Contains information on chemicals and } \\
\text { radionuclides after transfer of waste from } \\
\text { SY102 into AZ102. AZ102 supernatant was } \\
\text { then to be used as evaporator feed. } \\
\text { Outdated analysis. }\end{array}$ \\
\hline$A Z-102-9$ & $1 / 30 / 86$ & Sample Status Report dated 1/30/86. & Unavailable & $\begin{array}{l}\text { Slurped } \\
\text { sample }\end{array}$ & Reject & $\begin{array}{l}\text { Data table, no additional info. Outdated } \\
\text { analysis. }\end{array}$ \\
\hline$A Z-102-10$ & $2 / 20 / 86$ & $\begin{array}{l}\text { Mauss BM. 1986. 242-A Evaporator } \\
\text { Campaign 86-1: Laboratory Analyses. } \\
\text { Internal memo \# 65453-86-023 to RT } \\
\text { Kimura dated February 20, Rockwell } \\
\text { Hanford Operations, Richland, WA. }\end{array}$ & $12 / 2 / 85$ & Supernatant & Reject & $\begin{array}{l}\text { Analysis of AZ102 supernatant during the } \\
86-1 \text { evaporator campaign. Outdated } \\
\text { analysis. }\end{array}$ \\
\hline
\end{tabular}




\begin{tabular}{|c|c|c|c|c|c|c|}
\hline $\begin{array}{l}\text { Kaiser } \\
\text { Reference } \\
\text { Number }\end{array}$ & $\begin{array}{c}\text { Memo } \\
\text { Date }\end{array}$ & Source Documents* & $\begin{array}{c}\text { Sample } \\
\text { Date }\end{array}$ & Sample Type & $\begin{array}{l}\text { Accept/ } \\
\text { Reject }\end{array}$ & Comments \\
\hline AZ-102-11 & $5 / 6 / 86$ & $\begin{array}{l}\text { Mauss BM. 1986. Laboratory Analysis of } \\
\text { Tank 102-AZ Sample. Internal memo \# } \\
65453-86-066 \text { to TG Hanson dated 5/6/86, } \\
\text { Rockwell Hanford Operations, Richland, } \\
\text { WA. }\end{array}$ & $12 / 5 / 85$ & Sludge & Reject & $\begin{array}{l}\text { Analysis of both liquid and solid phases of a } \\
\text { centrifuged sludge sample were analyzed for } \\
\text { chemicals and radionuclides. Outdated } \\
\text { analysis. }\end{array}$ \\
\hline AZ-102-12 & $9 / 25 / 86$ & $\begin{array}{l}\text { Mauss BM, KE Schull. 1986. Analysis of } \\
\text { Samples from Pilot Plant NCAW Study. } \\
\text { Internal Letter } 65453-86-119 \text { to BD } \\
\text { Bullough dated 9/25/86, Rockwell Hanford } \\
\text { Operations, Richland, WA. }\end{array}$ & & & Reject & $\begin{array}{l}\text { It appears that there is no data specifically } \\
\text { from AZ102. There is data from simulated } \\
\text { pilot plant NCAW wastes. Does not pertain } \\
\text { to AZ102 directly. }\end{array}$ \\
\hline AZ-102-13 & $10 / 1 / 87$ & $\begin{array}{l}\text { Uziemblo NH, B Mastel, and RR Adee. } \\
\text { 1987. Characterization of Samples in } \\
\text { Support of Aging Waste Task Force. Letter } \\
\text { Report, Pacific Northwest Laboratory, } \\
\text { Richland, WA. }\end{array}$ & & $\begin{array}{l}\text { Supernatant/ } \\
\text { Sludge }\end{array}$ & Reject & $\begin{array}{l}\text { Contains only the summary of a much } \\
\text { longer report. Insufficient information to } \\
\text { evaluate. }\end{array}$ \\
\hline$A Z-102-14 a$ & $12 / 14 / 87$ & $\begin{array}{l}\text { Herting DL. 1987. Additional Information } \\
\text { on Analysis of Tank 102-AZ Samples Taken } \\
\text { in August, 1987. Internal memo \# 12221- } \\
\text { PCL-87-045 to LA Mihalik dated 12/14/87, } \\
\text { Westinghouse Hanford Company, Richland, } \\
\text { WA. }\end{array}$ & & Supernatant & Reject ' & $\begin{array}{l}\text { Contains data from AZ-102-14b, but doesn't } \\
\text { contain units on the data table. Outdated } \\
\text { analysis. }\end{array}$ \\
\hline$A Z-102-14 b$ & $12 / 8 / 87$ & $\begin{array}{l}\text { Herting DL. 1987. Chemical Analysis of } \\
\text { Tank 102-AZ Samples Taken in August } \\
\text { 1987. Internal memo \# 12221-PCL87-041 } \\
\text { to LA Mihalik dated 12/8/87, Westinghouse } \\
\text { Hanford Company, Richland, WA. }\end{array}$ & & Supernatant & Reject & $\begin{array}{l}\text { Contains data on supernatant and suspended } \\
\text { solids. The analysis for the solids was } \\
\text { corrected for the interstitial liquid. The } \\
\text { validity of this correction is questionable. } \\
\text { Outdated analysis. }\end{array}$ \\
\hline AZ-102-15 & $1 / 19 / 88$ & $\begin{array}{l}\text { Herting DL. 1987. Cold Test Results on } \\
\text { NCAW Sludge Volumes. Internal memo \# } \\
\text { 12221-PCL-87-065 to WW Schulz dated } \\
\text { 1/19/88, Westinghouse Hanford Company, } \\
\text { Richland, WA. }\end{array}$ & & & Reject & $\begin{array}{l}\text { All analysis done with simulated wastes. } \\
\text { No actual waste was used. Does not pertain } \\
\text { to AZ102 directly. }\end{array}$ \\
\hline
\end{tabular}




\begin{tabular}{|c|c|c|c|c|c|c|}
\hline $\begin{array}{c}\text { Kaiser } \\
\text { Reference } \\
\text { Number }\end{array}$ & $\begin{array}{l}\text { Memo } \\
\text { Date }\end{array}$ & Source Documents* & $\begin{array}{l}\text { Sample } \\
\text { Date }\end{array}$ & Sample Type & $\begin{array}{l}\text { Accept/ } \\
\text { Reject }\end{array}$ & Comments \\
\hline AZ-102-16 & $3 / 11 / 88$ & $\begin{array}{l}\text { Herting DL. 1988. Chemical Analysis of } \\
\text { Tank 101-AZ and 102-AZ Samples Taken in } \\
\text { October 1987. Internal memo \# } 12221- \\
\text { PCL88-091 to LA Mihalik dated 3/11/88, } \\
\text {-Westinghouse Hanford Company, Richland, } \\
\text { WA. }\end{array}$ & $10 / 87$ & $\begin{array}{l}\text { Supernatant / } \\
\text { Sludge }\end{array}$ & Reject & $\begin{array}{l}\text { Contains data on chemicals and } \\
\text { radionuclides for sludge and supernatant } \\
\text { samples. The validity of the method used } \\
\text { to determine the composition of the solid } \\
\text { phase is questionable. Outdated analysis. }\end{array}$ \\
\hline AZ-102-17 & $5 / 3 / 90$ & $\begin{array}{l}\text { McGrail BP. } 1990 \text { Preliminary Results - } \\
\text { Analyses of Waste Layers from DST 102-AZ } \\
\text { Core \#1. Internal Letter \# } 9002014 \text { to AJ } \\
\text { DiLiberto and LM Sasaki dated 5/3/90, } \\
\text { Pacific Northwest Laboratory, Richland, } \\
\text { WA. }\end{array}$ & Core & Sludge & Reject & $\begin{array}{l}\text { Contains information on three/four levels } \\
\text { from a two segment core (radionuclides and } \\
\text { chemicals). Information described } \\
\text { completely in AZ-102-23. }\end{array}$ \\
\hline AZ-102-18 & $11 / 1 / 90$ & $\begin{array}{l}\text { Gray WJ, ME Peterson, RD Scheele, JM } \\
\text { Tingey. 1990. Characterization of the } \\
\text { First Core Sample of Neutralized Current } \\
\text { Acid Waste from Double-shelled Tank 102- } \\
\text { AZ. Letter report \# } 9004292 \text { to NW Kirch } \\
\text { and LM Sasaki dated 11/1/90, Pacific } \\
\text { Northwest Laboratory, Richland, WA. }\end{array}$ & $7-8 / 89$ & Sludge & Reject & $\begin{array}{l}\text { Complete report is not given but gives info } \\
\text { on rheological properties and composition of } \\
\text { a composite sample from the } 2 \text { segment } \\
\text { core). Information described completely in } \\
\text { AZ-102-23. }\end{array}$ \\
\hline AZ-102-19 & $11 / 5 / 90$ & Report Analysis dated 11/5/90. & & Supernatant & Reject & $\begin{array}{l}\text { Data table only. References } 12711-90-016 \text {. } \\
\text { Insufficient information to evaluate. }\end{array}$ \\
\hline AZ-102-20 & $11 / 5 / 90$ & Report Analysis dated 11/5/90. & & Supernataṇt & Reject & $\begin{array}{l}\text { Data table only. References } 12711-90-016 \text {. } \\
\text { Insufficient information to evaluate. }\end{array}$ \\
\hline AZ-102-21 & $11 / 5 / 90$ & Report Analysis dated 11/5/90. & & Supernatant & Reject & $\begin{array}{l}\text { Data table only references } 12711-90-016 \text {. } \\
\text { Insufficient information to evaluate. }\end{array}$ \\
\hline$A Z-102-22$ & $11 / 5 / 90$ & Report Analysis dated 11/5/90. & & Supernatant & Reject & $\begin{array}{l}\text { Data table only references } 12711-90-016 \text {. } \\
\text { Insufficient information to evaluate. }\end{array}$ \\
\hline
\end{tabular}




\begin{tabular}{|c|c|c|c|c|c|c|}
\hline $\begin{array}{c}\text { Kaiser } \\
\text { Reference } \\
\text { Number }\end{array}$ & $\begin{array}{l}\text { Memo } \\
\text { Date }\end{array}$ & Source Documents* & $\begin{array}{c}\text { Sample } \\
\text { Date }\end{array}$ & Sample Type & $\begin{array}{c}\text { Accept/ } \\
\text { Reject }\end{array}$ & Comments \\
\hline$A Z-102-23$ & $9 / 93$ & $\begin{array}{l}\text { Gray WJ, ME Peterson, RD Scheele, JM } \\
\text { Tingey. 1993. Characterization of the } \\
\text { First Core Sample of Neutralized Current } \\
\text { Acid Waste From Double-shelled Tank 102- } \\
\text { AZ. Pacific Northwest Laboratory, } \\
\text { Richland, WA. }\end{array}$ & $5 / 89$ & Sludge & Accept & $\begin{array}{l}\text { Contains the complete analysis of the } 7-8 / 89 \\
\text { core sample. This document should be used } \\
\text { over AZ-102-17 and AZ-102-18. }\end{array}$ \\
\hline AZ-102-24 & $7 / 95$ & $\begin{array}{l}\text { Ryan GW. 1995. Tank Characterization } \\
\text { Report for Double-shelled Tank 241-AZ-102. } \\
\text { WHC-SD-WM-ER-411, Rev 0, } \\
\text { Westinghouse Hanford Company, Richland, } \\
\text { WA. }\end{array}$ & $5 / 89,2 / 95$ & . & Accept & Tank Characterization Report \\
\hline
\end{tabular}

* Many source documents are not cleared for public release. They are cited to provide traceablility to the original data. 


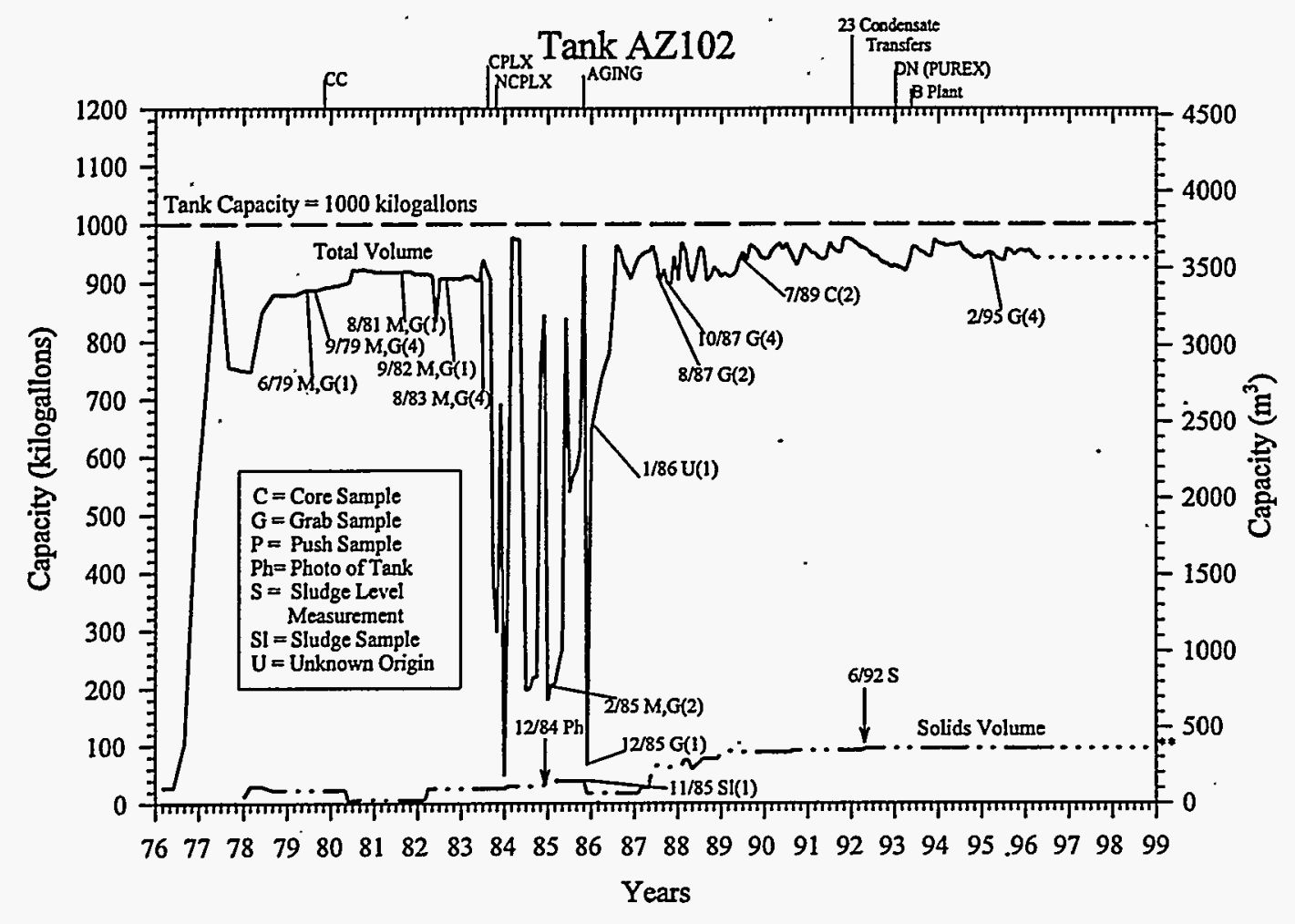

Figure 13.1. Tank Waste Volume and Sampling Event Graph for Tank AZ102

\section{6 $\mathrm{Min} / \mathrm{Max} / \mathrm{BE}$}

The minimum, maximum, and best estimate values were derived from February 1995 sampling data (AZ102-24) unless the data for a particular species were not available or consisted entirely of less than values. For these cases, supernatant data from June 1989 were used (AZ-10223).

\subsection{Organics}

The only organic analysis done on AZ102 was TOC.

\subsection{Physical Properties}

Extensive physical property measurements were made on the core sample taken from tank AZ102 in 1989. The measurements include density, percent solids, percent water, settling behavior, particle size, rheology, and penetration resistance. The results are summarized in the TCR for AZ102 (Ryan 1995b). Thermodynamic analyses (DSC and TGA) were performed on the 1995 samples (Ryan 1995b). 


\subsection{Limitations/Concerns}

The main limitation for the data used to generate the $\min / \max / \mathrm{BE}$ values for tank $\mathrm{AZ} 102$ is that some of the supernatant values are based on the centrate portion of the core sample taken in 1989. The composition of this interstitial liquid could be significantly different than the supernatant in the tank. However, it was used only when other data were not available.

The main concerns for tank AZ102 are the unexpectedly low nitrate/nitrite ratio and unexplained differences in the concentrations of nitrate, nitrite, and phosphate from those in AZ101. The nitrate/nitrite ratio is expected to be in the range of 2 to 3 ; however, the analysis of 1995 grab samples indicate that it is $\sim 1$. As for concentrations, both the 1989 core sample centrate and the 1995 grab samples indicate that the concentration of phosphate is $\sim 10$ times as high in AZ101 as in AZ102. Finally, the 1989 core sample centrate indicates the concentration of nitrate and nitrite are 10 times as high in AZ101 as in AZ102. 


\subsection{References}

Brevick CH, LA Gaddis, and ED Johnson. 1995. Tank Waste Source Term Inventory Validation. WHC-SD-WM-ER-400, prepared by ICF Kaiser Hanford Company for Westinghouse Hanford Company, Richland, Washington.

Brevick CH, LA Gaddis, and WW Pickett. 1995. Historical Tank Content Estimate for the Southeast Quadrant of the 200 East Areas. WHC-SD-WM-ER-350, prepared by ICF Kaiser Hanford Company for Westinghouse Hanford Company, Richland, Washington.

Certa'PJ. 1992. 242A Evaporator Crystallizer FY 84 Campaign Run 84-3 Post Run Document. WHC-SD-PE-018, Westinghouse Hanford Company, Richland, Washington.

DeLorenzo DS, AT DiCenso, DB Hiller, KW Johnson, JH Rutherford, DJ Smith, and BC Simpson. 1994. Tank Characterization Reference Guide. WHC-SD-WM-TI-648, Rev. 0, Westinghouse Hanford Company, Richland, Washington.

DOE. 1996a. TWRS Privatization Request for Proposals: Solicitation Number DE-RP06-96-RL 13308, U.S. Department of Energy, Richland, Washington.

DOE. 1996b. Amendment of Solicitation/Modification of Contract, Tanks Waste Remediation System (TWRS) Privatization. Amendment 001 to Solicitation DE-RP06-96-RL 13308, U.S. Department of Energy, Richland, Washington.

DOE. 1996c. Amendment of Solicitation/Modification of Contract, Tanks Waste Remediation System (TWRS) Privatization. Amendment 002 to Solicitation DE-RP06-96-RL 13308, U.S. Department of Energy, Richland, Washington.

Gratny EG. 1992. 242A Evaporator Crystallizer FY 84 Campaign Run 845 Post Run Document. WHC-SD-PE-022, Westinghouse Hanford Company, Richland, Washington.

Hodgson KM. 1995. Tank Characterization Report for Double-Shell Tank 241-AP-101. WHCSD-WM-ER-410. Westinghouse Hanford Company, Richland, Washington.

Hanlon BM. 1996. Waste Tank Summary Report for Month Ending April 30, 1996. WHC-EP0182-97. Westinghouse Hanford Company, Richland, Washington.

Johnson, ED. 1996. TWRS Privatization Support Project Waste Characterization Database Development. PNNL-10971, Pacific Northwest National Laboratory, Richland, Washington.

Koreski GM. 1995. Operational Waste Volume Projection. WHC-SD-ER-029, Rev 21. Westinghouse Hanford Company, Richland, Washington.

McKee RW, GS Anderson, MM King, WW Shultz, and TW Wood. 1995. Low-Activity Waste Envelopes for Phase I of the Hanford Tank Waste Remediation System Privatization Project. PNL10854. Pacific Northwest Laboratory, Richland, Washington. 
Patello GK, L Lauerhass, .RL Myers, H Thompson, and KD Wiemers. 1996. Low-Activity Waste Envelope Definition for the TWRS Privatization Phase I Request for Proposal, PNNL-11108, Pacific Northwest National Laboratory, Richland, Washington.

Patello GK. 1996. TWRS Privatization Support Project Waste Characterization Resource Dictionary, PNNL-10942. Pacific Northwest National Laboratory, Richland, Washington.

Ryan GW. 1995a. Tank Characterization Report for Double-Shell Tank 241-AY-102. WHC-SDWM-ER-454, Rev. 0, Westinghouse Hanford Company, Richland, Washington.

Ryan GW. 1995b. Tank Characterization Report for Double-Shell Tank 241-AZ-102. WHC-SDWM-ER-411, Rev 0, Westinghouse Hanford Company, Richland, Washington.

Shelton LW. 1996. Projected Double-Shell Tank Supernatant Composition and Inventory for Phase I Privatization. WHC-SD-WM-TI-751, Rev. 0, Westinghouse Hanford Company, Richland, Washington.

Welsh TL. 1991. Tank 241-AW-101 Characterization Test Results. WHC-SD-WM-TRP-055, Westinghouse Hanford Company, Richland, Washington.

Welsh TL. 1994a. Tank 241-AP-102 Characterization and Grout Product Test Results. WHCSD-WM-TRP-168, Westinghouse Hanford Company, Richland, Washington.

Welsh TL. 1994b. Tank 241-AP-105 Characterization Results. WHC-SD-WM-TRP-169, Westinghouse Hanford Company, Richland, Washington.

WHC. 1996. Operating Specification for the 241-AN, AP, AW AY, AZ and SY Tank Farms. OSD-T-101-00007, Westinghouse Hanford Company, Richland, Washington. 


\title{
Appendix A
}

\author{
Tank Sampling Methods
}




\section{Appendix A}

\section{Tank Sampling Methods}

Tank volume measurements are made with an automatic waste surface level measurement device (Hanlon 1996), manufactured by the Food Instrument Company (FIC). The device consists of a conductivity electrode connected to a calibrated steel tape, a steel tape reel housing, and a controller that automatically raises and lowers the plummet to obtain a waste surface level reading. FICs are in the process of being replaced by the ENRAF ATG 854 level detector manufactured by ENRAF, Incorporated. The ENRAF determines waste level by detecting variations in the weight of a displacer suspended in the tank waste. The displacer is connected to a wire wound onto a precision measuring drum. A level causes a change in weight of the displacer which will be detected by the force transducer. Electronics within the gauge causes the servo motor to adjust the position of the displacer and compute the tank level based on the new position of the displacer drum. This information is then recorded and transmitted to the Computer Automated Surveillance System (CASS).

In-tank photographs and videos aid in resolving in-tank measurement anomalies. Photographs and videos also help determine sludge and liquid levels by visual examination.

The types of samples removed from the tank are auger, grab, push; rotary, and vapor (De Lorenzo et al. 1994). Grab samples are the most common because they are the easiest and cheapest to obtain. Grab samples are obtained by placing a stoppered sampling bottle in a special cage and lowering it into the tank. At the desired tank level, the bottle is opened so that it fills with liquid; the full bottle is then restoppered and retrieved from the tank. Grab samples are used to obtain liquid or soft slurry samples.

Push sampling is a type of core sampling method used to obtain samples of solid and/or supernatant liquid high-level radioactive waste. Push samples are obtained from tanks containing liquids or soft sludge. Rotary sampling is a type of core sampling primarily used on sludge or hard salt cake, although it may be used to sample the other waste types. The samples are obtained with specially designed core sampling trucks and sampling devices. A cylindrical cross-section of the tank contents below the riser used for sampling is obtained by pushing or rotating a stainless steel sampler bit into the waste using a hydraulically powered drill string. A spring-loaded piston is used to create negative pressure which helps draw the sample into the waste column. Drill ram hydrostatic pressure is continuously monitored during the sampling operation to measure waste resistance. Core samples are taken repeatedly until the bottom of the tank is reached.

Auger samples are used to screen the surface of organic, ferrocyanide, and gas-generating watch list tanks, and may also' substitute for core sampling in tanks containing less than 20 to 30 $\mathrm{cm}$ of waste. A stainless steel, hand-turned auger bit contained in a sleeve is used in a manner similar to wood boring or ice augering. This sampling method has some significant limitations. Very fluid wastes cannot be properly captured in the sampler. The ability to check vertical 
variability is lost because the augering procedure disturbs the waste in the sample and affects recovery. Also, auger samples are limited to approximately the first $20 \mathrm{~cm}$ of solids on the tank waste surface.

There are three processes for obtaining vapor samples: a sample may be passed through a device that traps or concentrates certain vapors and gases, collected in an appropriate container, or analyzed in situ. The choice of the method for vapor sampling depends on the purpose of the sample as well as the type of vapor being sampled. 
Appendix B

Draft Tank Transfer Report from RS Goheen 


\title{
Appendix B
}

\section{Draft Tank Transfer Report from RS Goheen}

\author{
Recent Transfers for Double-Shelled Tanks \\ Draft Letter Report: Revision 2a
}

\author{
This document has not undergone an \\ independent review, and therefore the \\ accuracy of the information contained \\ herein has not been confirmed.
}

Robert S. Goheen

Last modification: August 1, 1995

This document lists the most recent major transfer of waste into or out of Hanford's underground double-shelled storage tanks (DST). This information was compiled to facilitate identification of analytical waste samples that do not represent the current composition of the waste. The most recent major transfer is listed, or if the tank has been receiving smaller volumes of waste over an extended duration, the change in the volume over a certain period of time is noted. The information in this document is based primarily on WHC-SD-WM-ER-350, Rev. 0, "Historical Tank Content Estimate for the Southeast Quadrant of the Hanford 200 East Area." Transfers made during 1994 and later were compiled from the monthly "Waste Tank Summary Report" (WHC-EP0182-72 through -84). Additional information is from the Tank Waste Information Network System (TWINS) electronic database.

This document describes the effect of transfers on total waste volume; it does not differentiate between solid and liquid volumes or between transfers of solids or liquids, except where specified. Additionally, the composition of the transfer is not investigated in detail. When available, the generating source of the waste or type of waste is listed (e.g., PUREX decladding supernate).

In addition to the volume of the transfer, a detailed analysis of the effect of a transfer on waste composition must consider other factors. For example, if a small transfer contains a high concentration. of one or more elements. it would drastically change the inventory of the waste, even though the concentration of other components might not change significantly. Also, reactions between the waste in the tank and any added waste could change the ratio of an element between the solid and liquid phases. Investigation into these factors would require significantly more information and analysis and is therefore beyond the scope of this document. 


\section{AN Farm}

\section{AN101}

This tank is designated as the East Area dilute non-complexed salt-well liquid-receiver tank. It is currently receiving waste. Approximately $160 \mathrm{kgal}$ of waste, $20 \%$ of the volume now in the tank, was added between March 1994 and March 1995.

\section{AN102}

The contents of AN102 (except for a small heel of $\sim 30 \mathrm{kgal}$ ) were transferred to tank AW102 in the second quarter of CY 1984. Approximately $1,080 \mathrm{kgal}$ of waste from AW101 was transferred in the second and third quarters of 1984. No further transfers have been made.

\section{AN103}

This tank is on the hydrogen watch list and is therefore prohibited from receiving waste. The last major transfer to this tank was in Q2 of 1986, approximately $650 \mathrm{kgal}$ of waste from tank AW102. That transfer represents approximately $70 \%$ of the waste volume now in the tank.

\section{AN104}

This tank is on the hydrogen watch list and is therefore prohibited from receiving waste. Transfers to this tank from AW102 in fourth quarter 1984 and second quarter 1985 totaled approximately $800 \mathrm{kgal}$ of waste and represent approximately $75 \%$ of the waste now in the tank. No transfers have been made since 1985 .

\section{AN105}

This tank has been essentially inactive since a large transfer $(\sim 1,000 \mathrm{kgal})$ of waste from AW102 in first quarter 1985. However, immediately before this transfer, the waste in AN105 was transferred to AW102. Therefore, the transfer to AN105 may not have affected the composition of the waste. About $500 \mathrm{kgal}$ was transferred from AN104 in second quarter 1984, and about 600 kgal was transferred from AW102 in fourth quarter 1982.

\section{AN106}

Essentially all the liquid was emptied from this tank in fourth quarter 1992. In September 1994, - $400 \mathrm{kgal}$ of slurry from the 94-1 Evaporator Campaign was transferred to this tank from AW106. That transfer accounts for all the now in AN105. It is expected that this tank will receive the slurry from the 95-1 Campaign.

\section{AN107}

This tank has been essentially inactive since two transfers of about $500 \mathrm{kgal}$ in 1983: from $\mathrm{AN} 102$ in the second quarter and from AZ102 in the third quarter. 


\section{AP Farm}

\section{AP101}

In fourth quarter 1994, the waste from this tank was sent to staging and then to the 242-B evaporator during the 94-2 campaign. Before the transfer, the tank contained $1060 \mathrm{kgal}$ of waste. During the latest evaporator run, waste was pumped from AP108 through this tank to pick up the waste heel.

\section{AP102}

During the second half of 1992, tank AP102 received approximately $950 \mathrm{kgal}$ of waste from tank AN106. This series of transfers accounts for $\sim 85 \%$ of the $1100 \mathrm{kgal}$ of waste in the tank.

\section{AP103}

This tank received two large transfers from AY102 ( $350 \mathrm{kgal}$ during Q4 1991 and $150 \mathrm{kgal}$ during Q2 1992). Approximately $1,100 \mathrm{kgal}(97 \%)$ of waste was removed from this tank and sent for staging for the 242-B evaporator during the.94-1 campaign.

\section{AP104}

Tank AP104 began to receive a total of approximately $450 \mathrm{kgal}$ of waste from SY102 in August 1995. AP104 first began to receive waste in fourth quarter 1986. In the first two quarters of 1987, the tank received approximately $950 \mathrm{kgal}$ of dilute phosphate waste from $100 \mathrm{~N}$ Area. In first quarter 1988 essentially all of the approximately $1070 \mathrm{kgal}$, in this tank was transferred to AP102, leaving about $20 \mathrm{kgal}$ of waste heel.

\section{AP105}

This tank has been inactive since Q3 of 1989. In 1987 through 1989, a series of transfers from AW102 led to a total volume in third quarter 1989, of approximately $475 \mathrm{kgal}$ of waste. AP105 then received $\sim 350 \mathrm{kgal}$ of waste from AP106. The waste volume has remained at approximately $825 \mathrm{kgal}$ since that transfer.

\section{AP106}

The contents of this tank was included in the 95-1 campaign of the 242-B evaporator. In July 1995 it contained approximately $100 \mathrm{kgal}$ of waste. The tank had been filled with waste from three other tanks: - $550 \mathrm{kgal}$ from AW106 in second quarter 1988, 120 shortly thereafter, and in second and third quarters 1989, two transfers of waste from AY102 ( $650 \mathrm{kgal})$.

\section{AP107}

Most of the waste in tank AP107 was evaporated during the 94-2 campaign. In September and October 1994, approximately $950 \mathrm{kgal}$ of waste was removed, leaving approximately $160 \mathrm{kgal}$. During the 95-1 campaign, waste from AW105 was pumped to AP108, then to AP101, then to 
AP107. Most of the waste from this transfer (quantity unknown) was pumped out of AP107 for evaporation. These transfers were done to pick up the waste heel left after the 94-2 campaign.

\section{AP108}

The waste in tank AP108 was evaporated during the 94-2 campaign. In September and October 1994, approximately $1,110 \mathrm{kgal}$ of waste was removed, leaving approximately $30 \mathrm{kgal}$. During the 95-1 campaign, waste from AW105 was pumped to AP108 and through AP101 and AP107 before being sent to the evaporator. After the waste from AW105 was pumped from AP108, this tank received waste from AP106, which was sent to the evaporator. These transfers were done to pick up the waste heel left after the 94-2 campaign.

\section{AW Farm}

\section{AW101}

Approximately $750 \mathrm{kgal}$ of waste was transferred from tank AW101 to AW102, which is the 242-B evaporator feed tank, from March to May 1986. Tank AW101 received approximately $1075 \mathrm{kgal}$ slurry from the evaporator in June and July 1986. No significant transfers have occurred since that time.

AW102

Tank AW102 is the 242-B evaporator feed tank and is active.

\section{AW103}

The last major transfers associated with this tank were recorded in 1988 and 1989. In third quarter 1987, approximately $160 \mathrm{kgal}$ PUREX decladding supernate and sludge was transferred to this tank; approximately $700 \mathrm{kgal}$ of PUREX waste and approximately $200 \mathrm{kgal}$ of water were added in 1988,. Three transfers of waste from AW103 to AW102 occurred in 1988 and 1989: approximately $400 \mathrm{kgal}$ during the PUREX transfers of second quarter 1988 approximately $100 \mathrm{kgal}$ in third quarter 1988, and $375 \mathrm{kgal}$ in first quarter 1989 . The tank has been inactive since these transfers.

\section{AW104}

Between January and June 1991, this tank received approximately $800 \mathrm{kgal}$ of mostly dilute and noncomplexed waste from PUREX. This tank has been inactive since this transfer.

\section{AW105}

This tank is currently active and receiving waste from PUREX. At the end of 1994, the pumpable liquid from this tank ( $\sim 730 \mathrm{kgal})$ was sent for staging and evaporation as a part of the 95-1 campaign. The total waste remaining in December 1994 was $342 \mathrm{kgal}$ (52 kgal pumpable liquid, $74 \mathrm{kgal}$ drainable liquid, $297 \mathrm{kgal}$ sludge). Between December 1994 and March 1995, addition of approximately $70 \mathrm{kgal}$ of liquid waste from PUREX increased the volume of total waste 
to approximately $410 \mathrm{kgal}$. This transfer represents approximately $60 \%$ of the liquid waste present in the tank as of March 1995. More liquid waste from PUREX has been added to the tank, but the exact volume added is not known. It is assumed that the solids in the tank have remained essentially constant (297 kgal) since 1987, when the last measurement was taken.

\section{AW106}

Tank AW106 is the slurry receiver tank. for the 242-B evaporator.

\section{$\mathrm{AY}$ and $\mathrm{AZ}$ Farms}

These four tanks are designated as aging waste tanks and were designed for a higher heat load than other DSTs. The tanks are equipped with condensers to handle evaporate. The condensate is collected in catch tanks and then either returned to one of the aging tanks or send to a dilute receiver tank. Water is added to these tanks to compensate for evaporative losses Therefore, all of these tanks have some small degree of current transfer activity related to the highheat nature of the waste.

Planned transfers to and from these four tanks should begin sometime in FY 1996 or FY 1997. These transfers, at this time, are considered to be independent from the Privatization effort. They correspond to the washing and consolidation of high-heat wastes in the AZ tanks and the pumping of solids from C106 to AY102.

The transfer of waste from C106 to AY102 is scheduled to begin in October 1996 and continue for six months to one year. The waste in $\mathrm{C} 106$ would be sluiced with buffered water and pumped to AY102. An early estimate is that approximately $500 \mathrm{kgal}$ of water will be needed to move the waste.

The planned start date for the washing and consolidation of high heat wastes was October 1995. The first operation would be to decant the liquids from AZ101 to AY101, and refill the tank with liquid AY102 or some other dilute waste liquid. The liquid from AZ102 would. be decanted and the solids from AZ101 transferred to AZ102. The retrieved waste from C106 (which would be in AY102 by this time) would also be transferred to AZ102. The washed solids would eventually be combined into one tank, and the washing solutions would be sent to other DSTs.

Although the dates and details of these transfers may change, or that the transfers be canceled or postponed, it is at this time highly likely that operations over the next two years will significantly impact the current composition of the waste in the AZ and AY tanks.

\section{AY101}

In May 1984, tank AY101 contain approximately $200 \mathrm{kgal}$ of dilute complexed waste. Between that date and June 1986, approximately $600 \mathrm{kgal}$ of dilute complexed waste from B-Plant was added to the tank. This tank, designated as the East Area dilute complexed salt-well receiver tank, received approximately $50 \mathrm{kgal}$ of waste from this activity between February and June of 1995. Evaporative losses and compensatory additions have also occurred. 
AY102

Tank AY102 is currently active and receiving dilute wastes from various sources. During the first half of 1995 , this tank received approximately $130 \mathrm{kgal}$ ( $\sim 15 \%$ of current volume) of waste, of which $\sim 90 \mathrm{kgal}$ is listed as flush water from various sources.

AZ101

In August 1983, tank AZ101 was essentially emptied of waste, except for a $-20 \mathrm{kgal}$ heel. From September 1983 until March 1986, this tank received approximately $800 \mathrm{kgal}$ of aging waste from the PUREX facility. A recorded addition of approximately $150 \mathrm{kgal}$ of water in June 1989 most likely reflects the total volume of several smaller additions over a longer period. Since then, the tank has been essentially inactive except for evaporative water losses and compensatory additions.

AZ102

In March 1986, tank AZ102 was essentially emptied of waste, except for a $\sim 20$ kgal heel. From April 1986 until January 1989, this tank received approximately $375 \mathrm{kgal}$ of aging waste from the PUREX facility. In June 1989, this tank received approximately $540 \mathrm{kgal}$ of water. Since that transfer, the tank has been essentially inactive except for evaporative water losses and compensatory additions.

\section{SY Farm}

\section{SY101}

The last major transfer to this tank was in November 1980. The volume of the tank has varied somewhat due to generation of gas and settling of solids.

SY102

This tank, designated as the West Area dilute non-complexed salt-well liquid receiver tank. It is receiving small transfers of waste from different sources: approximately $100 \mathrm{kgal}$ in 1993 and 1994 and approximately $30 \mathrm{kgal}$ in the first half of 1995 . In August 1995, approximately $450 \mathrm{kgal}$ of waste is scheduled to be transferred to tank AP104. Transfers over these three years represent $17 \%$ of the current waste volume in the tank.

SY103

The last recorded major transfer to this tank was during the second half of 1988, when approximately $150 \mathrm{kgal}$ ( $\sim 20 \%$ of the current volume of the tank) of dilute, non-complexed waste was added from the East Area SSTs. 
Table B.1. Summary of Transfer Information

\begin{tabular}{|c|c|c|c|}
\hline Tank & $\begin{array}{l}\text { Last Major } \\
\text { Transfer Date }\end{array}$ & Last Transfer Volume (kgal and \% of current vol.) and Notes & $\begin{array}{l}\text { Analytical } \\
\text { Data }\end{array}$ \\
\hline AN101 & $\begin{array}{l}\text { Currently } \\
\text { Active }\end{array}$ & $\begin{array}{l}160 \mathrm{kgal} \text { added ( } 20 \%) \text { between } 3 / 94 \text { and } 3 / 95 \text {. Tank is } \\
\text { designated as East Area dilute non-complexed salt well liquid } \\
\text { receiver tank. }\end{array}$ & \\
\hline AN102 & Q21984 & $\sim 1,000 \mathrm{kgal}$ added $(\sim 100 \%)$ from $A W 101$ & \\
\hline AN103 & Q4 1985 & $\sim 550 \mathrm{kgal}$ added $(60 \%)$ from $\mathrm{AW} 102$ & \\
\hline AN104 & Q2 1985 & $\sim 800 \mathrm{kgal}$ added $(75 \%)$ in 1984 and 1985 from $\mathrm{AW} 102$ & \\
\hline AN105 & Q1 1985 & $\sim 1,000 \mathrm{kgal}$ added $(\sim 100 \%)$ from $\mathrm{AW} 102$ & \\
\hline AN106 & Sept. 1994 & $\begin{array}{l}\sim 400 \mathrm{kgal} \text { of slurry from the } 94-1 \text { evaporator campaign added } \\
(\sim 100 \%)\end{array}$ & \\
\hline AN107 & Q3 1983 & $\sim 1,000 \mathrm{kgal}$ added $(\sim 100 \%)$ in 1982 and 1983. & \\
\hline AP101 & Mid 1995 & Contents sent to evaporator during 94-2 campaign. & \\
\hline AP102 & Q4 1992 & . & \\
\hline$\overline{A P 103}$ & May 1994 & Contents sent to evaporator during 94-1 .campaign. & \\
\hline AP104 & $\begin{array}{l}\text { Currently } \\
\text { Active }\end{array}$ & Receiving waste from SY102 & \\
\hline AP105 & Q31989 & & \\
\hline AP106 & May 1995 & Contents sent to evaporator during 95-1 campaign. & \\
\hline AP107 & May 1995 & Contents sent to evaporator during 94-2 campaign. & \\
\hline AP108 & May 1995 & Contents sent to evaporator during $94-2$ campaign. & \\
\hline AW101 & July 1986 & & \\
\hline AW102 & $\begin{array}{l}\text { Currently } \\
\text { Active }\end{array}$ & $\begin{array}{l}\text { Contents sent to evaporator during } 94-1 \text { campaign, is currently } \\
\text { used as feed tank for } 242-B \text { evaporator. }\end{array}$ & \\
\hline AW103 & Q4 1988 & $\begin{array}{l}\text { Received } \sim 860 \mathrm{kgal} \text { of PUREX cladding waste and } \sim 200 \mathrm{kgal} \\
\text { of water in } 1988 . \sim 875 \mathrm{kgal} \text { transferred to AW102 during } \\
\text { this time. }\end{array}$ & \\
\hline AWI04 & June 1991 & & \\
\hline AW105 & $\begin{array}{l}\text { Currently } \\
\text { Áctive }\end{array}$ & $\begin{array}{l}\text { Liquid portion of waste was pumped out and sent to the } \\
\text { evaporator. Receiving PUREX wastes. }\end{array}$ & \\
\hline AW106 & $\begin{array}{l}\text { Currently } \\
\text { Active }\end{array}$ & $\begin{array}{l}\text { Contents went to evaporator during } 94-1 \text { campaign, is currently } \\
\text { used as slurry receiver tank for } 242-\mathrm{B} \text { evaporator. }\end{array}$ & \\
\hline AY101 & $\begin{array}{l}\text { June } 1986 \\
\text { (Currently } \\
\text { Active) }\end{array}$ & $\begin{array}{l}\sim 600 \mathrm{kgal}(75 \%) \text { added between } 4 / 84 \text { and } 6 / 86.50 \mathrm{kgal} \text { of DC } \\
\text { waste from salt well pumping in first half } 1995 \text {. East Area } \\
\text { dilute complexed salt well liquid receiver tank. Aging waste } \\
\text { tank: water added and lost (evaporation). }\end{array}$ & \\
\hline AY102 & $\begin{array}{l}\text { Currently } \\
\text { Active }\end{array}$ & $\begin{array}{l}\sim 130 \text { kgal }(\sim 15 \%) \text { added during first half } 1995 \text {. Aging waste } \\
\text { tank: water added and lost (evaporation). Waste from this tank } \\
\text { is being transferred to AP104. }\end{array}$ & \\
\hline AZ101 & March 1986 & $\begin{array}{l}\sim 800 \mathrm{kgal}(\sim 100 \%) \text { added between } 9 / 83 \text { and } 3 / 86 . \sim 150 \\
\text { kgal }(\sim 16 \%) \text { of water added in } 6 / 89 . \text { Aging waste tank: water } \\
\text { added and lost (evaporation). }\end{array}$ & \\
\hline$\overline{A 2102}$ & January 1989 & $\begin{array}{l}\sim 375 \mathrm{kgal}(\sim 100 \%) \text { added between } 4 / 86 \text { and } 1 / 89 . \sim 550 \\
\text { kgal ( } \sim 60 \%) \text { of water added in } 6 / 89 . \text { Aging waste tank: water } \\
\text { added and lost (evaporation). }\end{array}$ & \\
\hline & & & \\
\hline
\end{tabular}

B. 7 


\begin{tabular}{|l|l|l|l|}
\hline SY101 & $\begin{array}{l}\text { November } \\
1980\end{array}$ & & \\
\hline SY102 & $\begin{array}{l}\text { Currently } \\
\text { Active }\end{array}$ & $\begin{array}{l}\text { This tank has been receiving small transfers from various } \\
\text { sources. From 1993 to June 1995, approximately 130 kgal has } \\
\text { been added, which is 17\% of the current total volume. West } \\
\text { Area dilute non-complexed salt well liquid receiver tank }\end{array}$ & \\
\hline SY103 & $\begin{array}{l}\text { December } \\
1988\end{array}$ & $\begin{array}{l}\text { l50 kgal ( 20\%) added between 6/88 and 12/88. Watch list } \\
\text { (hydrogen generation) }\end{array}$ & \\
\hline
\end{tabular}

Percentages are calculated as: (transfer vol.)/(total waste vol. after transfer) $\times 100 \%$. The total waste volume after the transfer is defined as the total listed tank volume after the specific transfer was completed. 


\section{Appendix C}

\section{Technical Reviewer Assignments}




\section{Appendix C}

\section{Technical Reviewer Assignments}

The table below shows the review assignments for the seven tanks for which current characterization information was available. The reviewer from Westinghouse Hanford Company (WHC) was DL Herting, the reviewer from Los Alamos National Laboratory (LANL) was JR FitzPatrick, and the lead reviewer from Argonne National Laboratory was GF Vandegrift.

Table C.1. Reviewer Tank Assignments

\begin{tabular}{||c|c|c|c||}
\hline Tank & ANL & LANL & WHC \\
\hline AN102 & $x$ & $x \dagger$ & \\
\hline AN107 & & $x$ & $x \dagger$ \\
\hline AP102 & $x$ & & \\
\hline AP105 & & & $x$ \\
\hline AW101 & & & $x$ \\
\hline AZ101 & & $x$ & \\
\hline AZ102 & $x$ & & \\
\hline AN103** & $x$ & & \\
\hline AN104* & $x$ & & \\
\hline AN105* & $x$ & & \\
\hline AY102** & $x$ & & \\
\hline C106** & $x$ & & \\
\hline
\end{tabular}

* These tanks had no current characterization data but tank references were reviewed independent of Kaiser's data collection effort to determine if any relevant information could be attained.

** The need for information on these tanks was not identified in time to be include on the Kaiser statement of work. The data for these tanks were collected independently from Kaisers work from original references. $\dagger$ Determined minimum, maximum, and best estimate values from the Subset only as a check.

C.1 


\section{Distribution}

No. of

Copies

OFFSITE

2 DOE/Office of Scientific and Technical Information

G. F. Vandegrift

Argonne National Laboratory

9700 South Cass Avenue

Argonne, IL 60439-4837

C. J. Conner

Argonne National Laboratory

9700 South Cass Avenue

Argonne, IL 60439-4837

C. J. Mertz

Argonne National Laboratory

9700 South Cass Avenue

Argonne, IL 60439-4837

M. C. Regalbuto

Argonne National Laboratory

9700 South Cass Avenue

Argonne, IL 60439-4837

L. Chen

Argonne National Laboratory

9700 South Cass Avenue

Argonne, IL 60439-4837

J. R. FitzPatrick

CST-7 G739

Los Alamos National Laboratory

Los Alamos, NM 87545
No. of

Copies

ONSITE

Westinghouse Hanford Company

D. L. Herting

T6-09

2 Kaiser Hanford Company

C. H. Brevick

S3-10

E. D. Johnson

S3-09

2 Department of Energy

N. R. Brown

K6-51.

R. A. Gilbert

K6-51

17 Pacific Northwest National Laboratory

L. K. Holton

K9-73

R. L. Myers

P7-20

G. K. Patello (2)

P7-28

R. L. Russell

P7-28

K. R. Savard

K9-04

D. Vela (2)

K6-51

K. D. Wiemers (2)

K6-51

Information Release Office (7) K1-06

Distr.1 